\title{
ANALYTICAL AND EXPERIMENTAL STUDY OF CONTROL EFFORT ASSOCIATED WITH MODEL REFERENCE ADAPTIVE CONTROL
}

by

\author{
Richard Scott Messer \\ Dissertation Submitted to the Faculty of \\ Virginia Polytechnic Institute and State University \\ in partial fulfillment of the requirements for the degree of \\ Doctor of Philosophy \\ in \\ Aerospace Engineering
}

APPROVED:

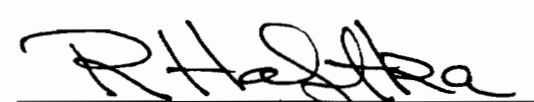

Raphael T. Haftka

Co-Chairman

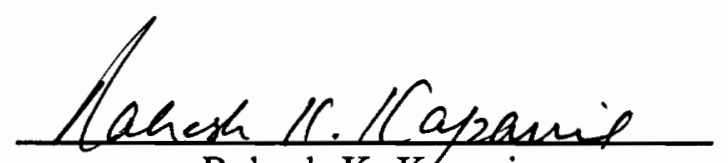

Rakesh K. Kapania

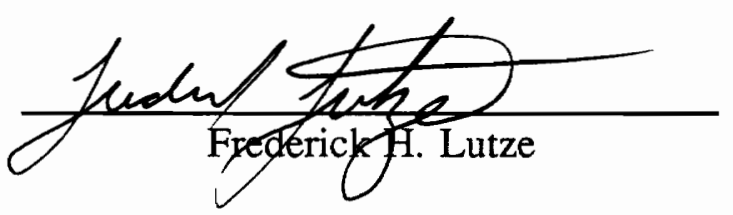

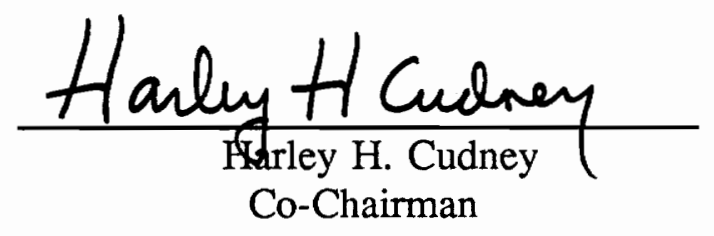
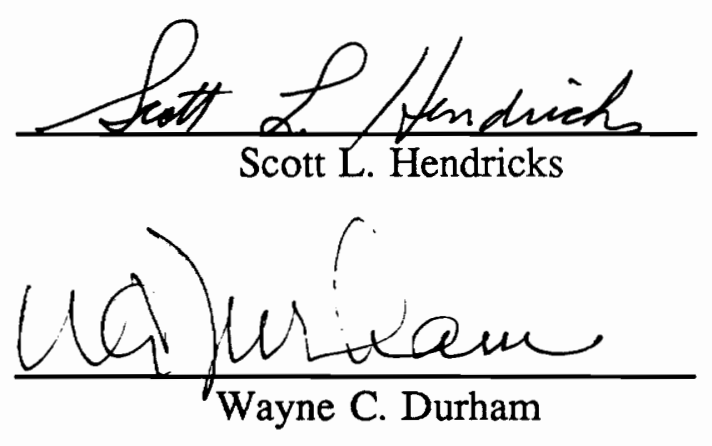

July 1992

Blacksburg, Virginia 


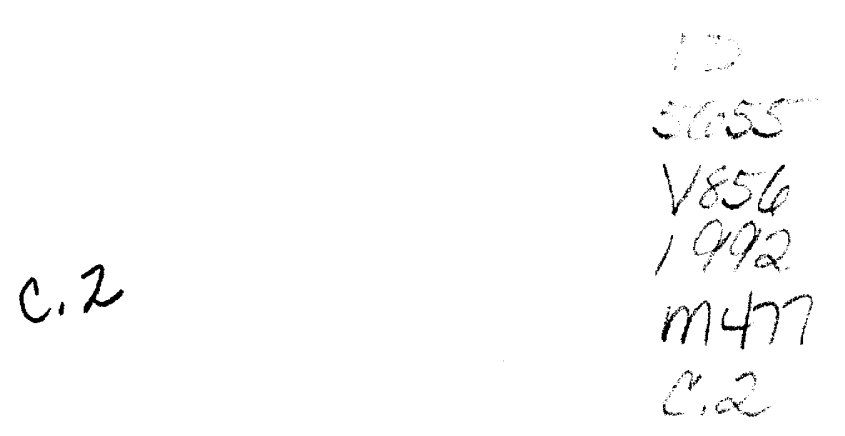




\title{
ANALYTICAL AND EXPERIMENTAL STUDY OF CONTROL EFFORT ASSOCIATED WITH MODEL REFERENCE ADAPTIVE CONTROL
}

\author{
BY \\ Richard Scott Messer \\ Raphael T. Haftka \\ Harley H. Cudney
}

(ABSTRACT)

During the past decade, researchers have shown much interest in control and identification of Large Space Structures (LSS). Our inability to model these LSS accurately has generated extensive research into robust controllers capable of maintaining stability in the presence of large structural uncertainties as well as changing structural characteristics. In this work the performance of Model Reference Adaptive Control (MRAC) is studied in numerical simulations and verified experimentally, to understand how differences between the piant and the reference model affect the control effort. MRAC is applied analytically and experimentally to a single-degree-of-freedom system and analytically to a multi-degree-of-freedom system with multi-inputs and multi-outputs. Good experimental and analytical agreement is demonstrated in control experiments and it is shown that MRAC does an excellent job of controlling the structures and achieving the desired performance even when large differences between the plant and ideal reference model exist. However, it is shown that reasonable differences between the reference model and the plant significantly increase the required control effort.

The effects of increased damping in the reference model are considered, and it is shown that requiring the controller to provide increased damping actually decreases the required control effort when differences between the plant and reference model exist. This result is very useful because one of the first attempts to counteract the increased 
control effort due to differences between the plant and reference model might be to require less damping, however, this would actually increase the control effort.

The use of optimization to successfully improve performance and reduce control effort is shown to be limited, because the actual control-structure system can not realize all the performance improvements of the analytical optimal system. Finally, it is shown that very large sampling rates may be required to accurately implement MRAC. 


\section{Acknowledgements}

I am greatly indebted to my present committee co-chairmen Dr. Raphael T. Haftka and Dr. Harley H. Cudney, and to my past committee co-chairmen Dr. William L Hallauer Jr., for their expertise, patience, and guidance through the course of my graduate studies. It has been my great pleasure to have been taught by such great men. Dr. Haftka provided the guiding hand that steered the analytical side of the research while Dr. Cudney and Dr. Hallauer provided a sound basis for the experimental work.

I would like to thank my committee members Dr. Rakesh K. Kapania, Dr. Scott Hendricks, Dr. Fred Lutze, and Dr. Wayne Durham for their time and consultation throughout the years.

I appreciate the support of NASA Langley Research Center for the grant NAG-1-224 which supported this work in part. Thanks also goes to Dr. Haftka and Dr. Schetz for their continued efforts to make sure some type of funding was always available.

Thanks are due to the staff of the Aerospace and Ocean Engineering Department particularly the office staff, Betty, Gay, Pat, and Sandy, the shop staff, Frank, Jake, Kent and Bruce, and finally Gary Stafford without whose help I would have never made it through.

Finally, I wish to express my love and thanks to my wife and family for their support, love, encouragement, and patience with the many long hours. Without their love and support this work would have been impossible. 


\section{Table of Contents}

I Introduction and Background $\ldots \ldots \ldots \ldots \ldots \ldots \ldots \ldots \ldots \ldots$ I

1.1 Large Space Structures and Robust Control $\ldots \ldots \ldots \ldots \ldots \ldots \ldots \ldots$

1.2 Model Reference Adaptive Control $\ldots \ldots \ldots \ldots \ldots \ldots \ldots \ldots \ldots \ldots 4$

1.3 Objectives $\ldots \ldots \ldots \ldots \ldots \ldots \ldots \ldots \ldots \ldots \ldots \ldots \ldots, 8$

1.4 Approach $\ldots \ldots \ldots \ldots \ldots \ldots \ldots \ldots \ldots \ldots \ldots \ldots \ldots \ldots$

II Model Reference Adaptive Control $\ldots \ldots \ldots \ldots \ldots \ldots \ldots \ldots \ldots \ldots$

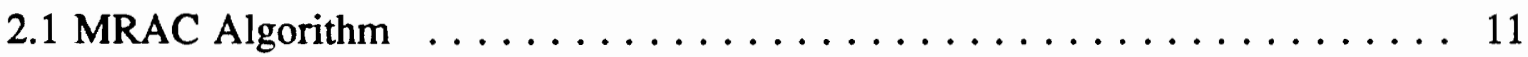

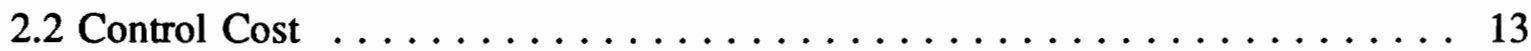

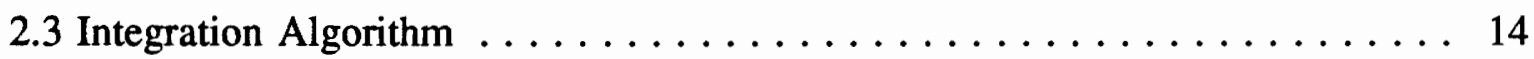

2.3.1 Experimental Implementation $\ldots \ldots \ldots \ldots \ldots \ldots \ldots \ldots \ldots$

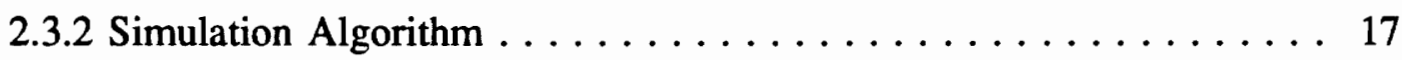


III Example Structures: Analytical Models and Experimental Set Up . . . 20

3.1 Structural Models . . . . . . . . . . . . . . . . . . . 20

3.1.1 Suspended Mass System . . . . . . . . . . . . . . 21

3.1 .2 Slewing Grid Structure $\ldots \ldots \ldots \ldots \ldots \ldots \ldots \ldots \ldots$

3.2 Choosing the Reference Model $\ldots \ldots \ldots \ldots \ldots \ldots \ldots \ldots \ldots \ldots$

3.2.1 Suspended Mass Reference Model . . . . . . . . . . . 25

3.2.2 Slewing Grid Reference Model $\ldots \ldots \ldots \ldots \ldots \ldots \ldots \ldots$

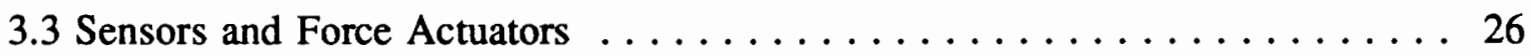

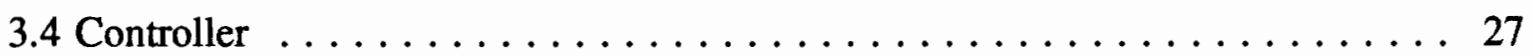

3.5 Experimental Set Up and Procedure $\ldots \ldots \ldots \ldots \ldots \ldots \ldots \ldots \ldots$

IV Effects of Error on MRAC Performance and Control Effort $\ldots \ldots \ldots 52$

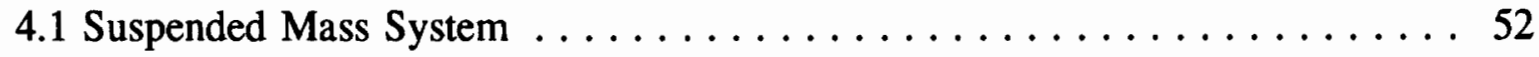

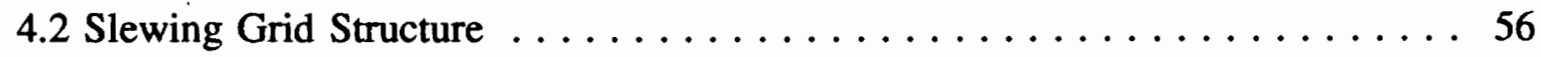

V Weighting Matrix Optimization $\ldots \ldots \ldots \ldots \ldots \ldots \ldots \ldots \ldots \ldots 77$

5.1 Measure of Fit for Suspended Mass System . . . . . . . . . . . 77

5.2 Choice of Design Variables $\ldots \ldots \ldots \ldots \ldots \ldots \ldots \ldots \ldots \ldots \ldots$

$\begin{array}{ll}\text { Table of Contents } & \text { vi }\end{array}$ 
5.3 Optimization Formulation $\ldots \ldots \ldots \ldots \ldots \ldots$

5.4 Optimization Results Using Only Diagonal Elements $\ldots \ldots \ldots \ldots \ldots$

5.5 Optimization Results Using Off Diagonal Elements $\ldots \ldots \ldots \ldots \ldots 1$

5.6 Optimization Results Using Diagonal Elements and Damping Ratios . . . . . 84

VI Concluding Remarks $\ldots \ldots \ldots \ldots \ldots \ldots \ldots \ldots \ldots \ldots$

Appendix A: Derivation of Integration Algorithm $\ldots \ldots \ldots \ldots \ldots \ldots 110$

Appendix B: Slewing Grid: Description and Finite Element Analysis . . . 112

B.1 Slewing Grid Description $\ldots \ldots \ldots \ldots \ldots \ldots \ldots \ldots \ldots \ldots \ldots$

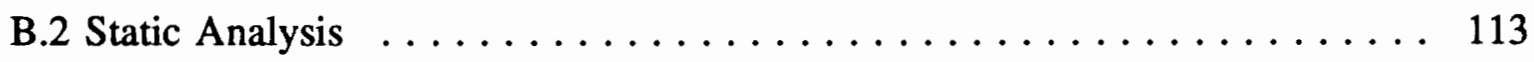

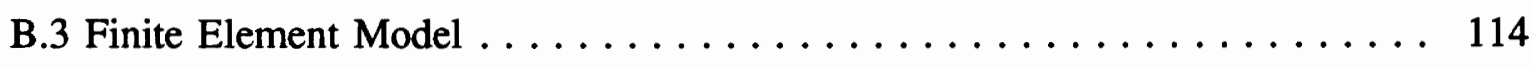

B.4 Experimental/Analytical Modal Analysis Results . . . . . . . . . 115 


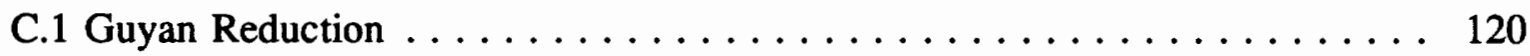

C.2 Reduced Model Correction $\ldots \ldots \ldots \ldots \ldots \ldots \ldots \ldots \ldots \ldots \ldots \ldots$

C.3 Reduction and Correction Procedures and Results $\ldots \ldots \ldots \ldots \ldots \ldots 122$

Appendix D: Sensor and Actuator Calibration $\ldots \ldots \ldots \ldots \ldots \ldots \ldots$

D.1 Theory . . . . . . . . . . . . . . . . . . . . . . . 129

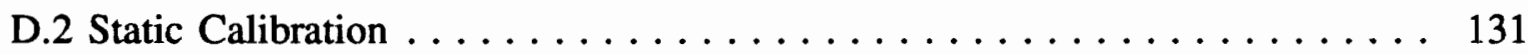

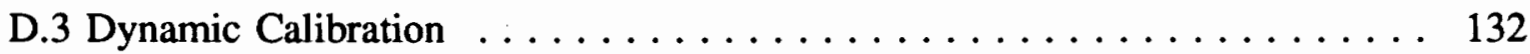

D.4 Comparison of Static and Dynamic Calibrations $\ldots \ldots \ldots \ldots \ldots \ldots$

References $\ldots \ldots \ldots \ldots \ldots \ldots \ldots \ldots \ldots \ldots \ldots \ldots \ldots \ldots \ldots \ldots$

Vita.$\ldots \ldots \ldots \ldots \ldots \ldots \ldots \ldots \ldots \ldots \ldots \ldots \ldots \ldots \ldots \ldots$ 


\section{List of Tables}

Table 3-1 Comparison of Analytical and Experimental Frequencies for the Slewing Grid Structure . . . . . . . . . . . . . 30

Table 3-2 Measured and Desired Damping Ratios for the Slewing Grid

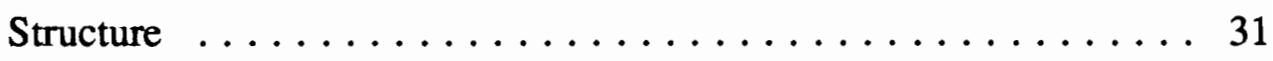

Table 4-1 Start Times (sec) for the Two Natural Frequencies That Most Tests Were Run at (Suspended Mass) . . . . . . . . . . . . . . 59

Table 4-2 Comparison of Experimental and Analytical Maximum Force and Total Control Effort (Suspended Mass) . . . . . . . . . . 60

Table 4-3 Analytical Control Effort and Maximum Force for the Slewing Grid. Initial Conditions $=$ Second Mode Shape $\ldots \ldots \ldots 1$

Table 4-4 Analytical Control Effort and Maximum Force for the Slewing Grid. Initial Conditions $=$ Fourth Mode Shape $\ldots \ldots \ldots 2$

Table 4-5 Analytical Control Effort and Maximum Force for the Slewing Grid at Maximum Difference $(\alpha=0.0)$ Between the Plant and Reference Model Initial Conditions $=$ Second Mode Shape ... . 63

Table 5-1 Initial and Optimal Designs of The Diagonal elements For the $\mathrm{T}$ and $\mathrm{T}^{*}$ Matrices (Suspended Mass System) $\ldots \ldots \ldots 6$

Table 5-2 Derivatives of the Maximum Force With Respect to Off Diagonal Terms of the $\mathrm{T}$ and $\mathrm{T}^{*}$ Matrices, Evaluated at the Optimal Diagonal Design .......................... 87

Table 5-3 Initial and Optimal Designs with off Diagonal Elements of The $T$ and $\mathrm{T}^{*}$ Matrices (Suspended Mass System) . . . . . . . 88

Table 5-4 Experimental/Analytical Comparison of The Maximum Force and Fitness (Suspended Mass System, $\alpha=0.5$ ) . . . . . . . . 89 
Table C-1 Comparison of Frequencies for the Reduced 11 DOF System With the Full Model 72 DOF (Slewing Grid) . . . . . . . . . . . . 125

Table C-2 Comparison of Corrected Model Frequencies as the Model is Corrected One Mode at a Time (Slewing Grid) . . . . . . . 126 


\section{List of Illustrations}

Figure 2-1 Model Reference Adaptive Control Block Diagram . . . . . . . . . 19

Figure 3-1 Drawing of Suspended Mass System . . . . . . . . . . . 32

Figure 3-2 Analytical Model of the Suspended Mass System . . . . . . . . 33

Figure 3-3 Magnitude and Phase Plot of a Velocity Frequency Response Function for the Suspended Mass System . . . . . . . . . . . 34

Figure 3-4 Comparison of Analytical and Experimental Free Velocity Response for the Suspended Mass System, Top: $0.1 \mathrm{~Hz}$ Difference between Experimental and Analytical frequency Bottom: Experimental $=$ Analytical Frequency $\ldots \ldots \ldots 35$

Figure $3-5$ Drawing of the Slewing Grid $\ldots \ldots \ldots \ldots \ldots$

Figure 3-6 Comparison of the Analytical and Experimental First Mode Shape for the Slewing Grid ................. 37

Figure 3-7 Comparison of the Analytical and Experimental Second Mode Shape for the Slewing Grid $\ldots \ldots \ldots \ldots$. . . . . . . . 38

Figure 3-8 Comparison of the Analytical and Experimental Third Mode Shape for the Slewing Grid . . . . . . . . . . . . . . . 39

Figure 3-9 Comparison of the Analytical and Experimental Fourth Mode Shape for the Slewing Grid $\ldots \ldots \ldots \ldots \ldots$

Figure 3-10 Comparison of the Analytical and Experimental Fifth Mode Shape for the Slewing Grid $\ldots \ldots \ldots \ldots \ldots \ldots \ldots$

Figure 3-11 Comparison of the Analytical and Experimental Seventh Mode Shape for the Slewing Grid

Figure 3-12 Comparison of the Analytical and Experimental Eighth Mode Shape for the Slewing Grid $\ldots \ldots \ldots \ldots \ldots$ 
Figure 3-13 Sketch of Displacement Probe by Bently Nevada . . . . . . . . 44

Figure 3-14 Sketch of the Coil and Magnetic Field Assembly Used by Velocity Sensor and Force Actuators $\ldots \ldots \ldots \ldots \ldots \ldots \ldots \ldots$

Figure 3-15 Diagram of Force Direction with Respect to the Coil and Magnetic

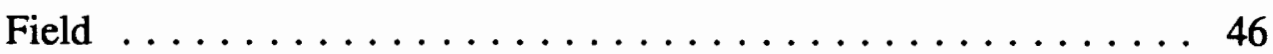

Figure 3-16 Block Diagram of the Experimental Set up for the Suspended Mass .......................... 47

Figure 3-17 Schematic of Electrical Circuits Used for Velocity Signal . . . . 48

Figure 3-18 Schematic of Electrical Circuits Used for Displacement Signal . . . 49

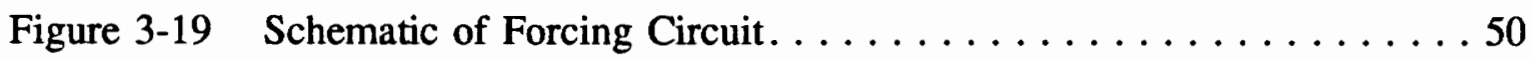

Figure 3-20 Actuator Response when DC Voltage is Set to Zero . . . . . . . 51

Figure 4-1 Analytical Maximum Force (Lbs) and Initial Velocity (in/sec) for the Suspended Mass Versus Start Time $(\alpha=1.0) \ldots \ldots \ldots \ldots 64$

Figure 4-2 Analytical Maximum Force (lbs) and Initial Velocity (in/sec) for Suspended Mass Versus Start Time $(\alpha=0.5) \ldots \ldots \ldots \ldots \ldots 65$

Figure 4-3 Analytical Maximum Force (lbs) and Initial Velocity (in/sec) for Suspended Mass Versus Start Time $(\alpha=1.5) \ldots \ldots \ldots \ldots \ldots 66$

Figure 4-4 Effect of Damping on Maximum Force for Varying Amounts of Frequency Difference (Suspended Mass) . . . . . . . . . . . 67

Figure 4-5 Effect of Damping on Control Effort for Varying Amounts of Frequency Difference (Suspended Mass) $\ldots \ldots \ldots \ldots \ldots \ldots 68$

Figure 4-6 Comparison of Experimental and Analytical Velocity Response of the Suspended Mass System, $\omega_{\mathrm{nm}}=1.0 \omega_{\mathrm{np}} \ldots \ldots \ldots \ldots \ldots 69$

Figure 4-7 Comparison of Experimental and Analytical Velocity Response of the Suspended Mass System, $\omega_{\mathrm{nm}}=1.3 \omega_{\mathrm{np}} \ldots \ldots \ldots \ldots \ldots$

Figure 4-8 Comparison of Experimental and Analytical Velocity Response of the Suspended Mass System, $\omega_{\mathrm{nm}}=0.7 \omega_{\mathrm{np}} \ldots \ldots \ldots \ldots \ldots \ldots$ 
Figure 4-9 Comparison of Analytical and Experimental Maximum Force Magnification Factor for the Suspended Mass $\ldots \ldots \ldots \ldots \ldots 72$

Figure 4-10 Comparison of Analytical and Experimental Control Effort Magnification Factor for the Suspended Mass $\ldots \ldots \ldots \ldots .73$

Figure 4-11 Analytical Maximum Force Magnification Factor for the Slewing Grid $($ Initial Displacement $=$ Analytical Second Mode $) \ldots \ldots$. . 74

Figure 4-12 Analytical Maximum Force Magnification Factor for the Slewing Grid (Initial Displacement is Analytical Fourth Mode) . . . . . . 75

Figure 4-13 Analytical Control Effort Magnification Factor for the Slewing Grid ......................... 76

Figure 5-1 Unshifted Analytical Velocity Response of The Suspended Mass System $\left(\alpha=0.5, T=50.0[\mathrm{I}]\right.$, and $\left.\mathrm{T}^{*}=[\mathrm{I}]\right) \ldots \ldots \ldots \ldots$

Figure 5-2 Unshifted Analytical Velocity Response of The Suspended Mass System $\left(\alpha=1.5, T=50.0[\mathrm{I}]\right.$, and $\mathrm{T}^{*}=[\mathrm{I}) \ldots \ldots \ldots \ldots \ldots$

Figure 5-3 Shifted $\left(t_{d}=0.03 \mathrm{sec}\right)$ Analytical Velocity Response of The Suspended Mass System $\left(\alpha=0.5, T=50.0[1]\right.$, and $\left.T^{*}=[1]\right) \ldots 92$

Figure 5-4 Shifted $\left(t_{d}=0.01 \mathrm{sec}\right)$ Analytical Velocity Response of The Suspended Mass System $\left(\alpha=1.5, T=50.0[1]\right.$, and $\left.T^{*}=[I]\right) \ldots 93$

Figure 5-5 Analytical Maximum Force For Different Diagonal $\mathrm{T}$ and $\mathrm{T}^{*}$ $T=\lambda[I], T^{*}=[\mathrm{I}]$ or $\mathrm{T}=16 \mathrm{I}, \mathrm{T}^{*}=\lambda \mathrm{I} \quad$ (Suspended Mass System, $\alpha=1) \ldots \ldots \ldots \ldots \ldots \ldots \ldots$

Figure 5-6 Analytical Fit For Different Diagonal $T$ and $T^{*} T=\lambda\left[\Pi, T^{*}=[I]\right.$ or $\mathrm{T}=16 \mathrm{I}, \mathrm{T}^{*}=\lambda \mathrm{I} \quad$ (Suspended Mass System, $\left.\alpha=1\right) \ldots \ldots \ldots 95$

Figure 5-7 Analytical Maximum Force For Different Diagonal $T$ and $T^{*}$ $T=\lambda\left[\Pi, T^{*}=[\Pi\right.$ (Suspended Mass System, $\alpha=1) \ldots \ldots \ldots 96$

Figure 5-8 Analytical Fit For Different Diagonal $T$ and $T^{*} T=\lambda[I], T^{*}=[I]$ (Suspended Mass System, $\alpha=1$ ) . . . . . . . . 97 
Figure 5-9 Experimental Maximum Force For Different Diagonal $\mathrm{T}$ and $\mathrm{T}^{*}$ $\mathrm{T}=\lambda\left[\Pi, \mathrm{T}^{*}=[\Pi\right.$ (Suspended Mass System, $\alpha=1) \ldots \ldots \ldots 98$

Figure 5-10 Experimental Maximum Force For Different Values of Alpha $\mathrm{T}=50[\mathrm{I}], \mathrm{T}^{*}=[\mathrm{I}]$ (Suspended Mass System) .

Figure 5-11 Experimental Fit For Different Values of Alpha $T=50\left[\mathrm{I}, \mathrm{T}^{*}=[\mathrm{I}]\right.$ (Suspended Mass System) . . . . . . . . . . . . . . . 100

Figure 5-12 Experimental and Analytical Maximum Force For Intermediate Design Points Between Case 2 Optimal Design, and Case 3 Optimal Design (Suspended Mass System) . . . . . . . . . 101

Figure 5-13 Experimental and Analytical Maximum Force For Intermediate Design Points Between Case 2 Optimal Design, and Case 3 Optimal Design (Suspended Mass System) . . . . . . . . . . 102

Figure 5-14 Experimental \& Analytical Maximum Force for Varying Sizes of the Off Diagonal Elements $T^{*}(1,2) \& T^{*}(2,1)$, and Sampling Rates (Suspended Mass System, $\alpha=1.5$ ) $\ldots \ldots \ldots \ldots \ldots \ldots \ldots$

Figure 5-15 Experimental \& Analytical Maximum Force for Varying Sizes of the Off Diagonal Elements $T^{*}(1,2) \& T^{*}(2,1)$, and Sampling Rates (Suspended Mass System, $\alpha=0.5$ ) . . . . . . . . . 104

Figure 5-16 Experimental \& Analytical Fit for Varying Sizes of the Off Diagonal Elements $\mathbf{T}^{*}(1,2) \& T^{*}(2,1)$, and Sampling Rates

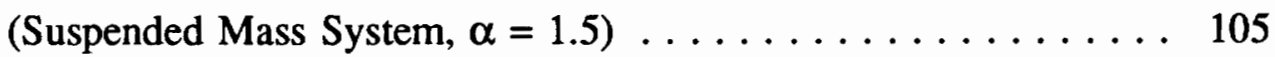

Figure 5-17 Analytical Maximum Force for Different Diagonal $T^{*} T=16[I]$, $T^{*}=\lambda[I]$ (Suspended Mass System $\left.\alpha=1\right) \ldots \ldots \ldots \ldots \ldots$

Figure 5-18 Experimental and Analytical Effects of Damping on the Maximum

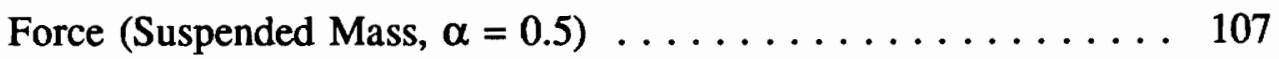

Figure 5-19 Experimental and Analytical Effects of Damping on the Maximum Force (Suspended Mass, $\alpha=1.5) \ldots \ldots \ldots \ldots \ldots \ldots$ 
Figure B-1 Configuration of The Strain Gages Mounted on Members of The Slewing Grid to Calculate Forces Due to Tightening of Bolts . . . 117

Figure B-2 Numbering Scheme For The Finite Element Model of the Slewing Grid Structure $\ldots \ldots \ldots \ldots \ldots \ldots \ldots \ldots \ldots \ldots \ldots \ldots \ldots \ldots \ldots$

Figure B-3 Grid Element and Related Degrees of Freedom . . . . . . . . . . 119

Figure C-1 Degrees of Freedom for the Slewing Grid reduced Model . . . . 127

Figure C-2 Sum of Elements in the Reduced Mass Matrix as the Number of Modes Applied to the Correction Process is Increased . . . . . 128

Figure D-1 Sketch of a Wire with Current in a Magnetic Field . . . . . . . 135

Figure D-2 Sketch of a Wire Moving Through a Magnetic Field . . . . . . . 136

Figure D-3 Static Calibration Set Up . . . . . . . . . . . . . . 137

Figure D-4 Markings on Coil for Depth Tests . . . . . . . . . . . 138

Figure D-5 Static Calibration Curve (Force Actuator) . . . . . . . . . . 139

Figure D-6 Static Calibration Curve (Velocity Sensor) . . . . . . . . . 140

Figure D-7 Dynamic Calibration Set Up . . . . . . . . . . . . 141

Figure D-8 Dynamic Calibration Data (Force Actuator) $\ldots \ldots \ldots \ldots \ldots .142$

Figure D-9 Dynamic Calibration Data (Velocity Sensor) . . . . . . . . . 143 


\section{Chapter 1}

\section{Introduction and Background}

During the past decade, researchers have shown much interest in control and identification of Large Space Structures (LSS). Our inability to model these LSS accurately has generated extensive (see ref. 1-4) research into controllers capable of maintaining stability in the presence of large structural uncertainties as well as changing structural characteristics. In this chapter we discuss the need for robust control and present a brief overview of some of the non-adaptive and adaptive methods for controlling LSS.

\subsection{Large Space Structures and Robust Control}

Research into robust control has focused on two types of controllers, robust nonadaptive controllers which do not update controller gains during operation, and adaptive controllers which constantly update controller gains to maximize design objectives. Examples of robust non-adaptive control approaches include techniques like $\mathrm{H}$-infinity (e.g., refs. 5-6), restoring robustness to the classical Linear-Quadratic Gaussian (LQG) (e.g., loop transfer recovery, ref. 7), optimizing measures of system robustness while specifying bounds on the eigenvalues (e.g., refs. 8-10), or using inherently robust simple controllers such as direct-rate feedback (e.g., refs. 11 and 12).

The question, which of the two types of robust control to use, can not be answered for every application. In fact, it is likely that a combination of both types of controllers will be used. Present challenges such as stringent performance requirements and difficulties in modeling indicate that adaptive controllers will be required for many 
applications. Challenges such as time delays, electrical circuit non-linearities, friction, non-linear structural behavior, and joint backlash are all examples of what makes accurate modeling of real structures difficult. Even if all these difficulties are overcome, it is unlikely that the response of the structure in a gravity free environment will be the same as measured in an earth based laboratory. In fact, it was these types of challenges that caused researchers to consider adaptive control.

For example, the Slewing Grid structure, a laboratory structure at Virginia Tech, (discussed in chapter 3), has proven to be very difficult to model. Efforts to improve the model included studies to better understand the physical characteristics of the structure, system identification, and model correction based upon experimental measurements. However, the behavior of the mathematical model is still very different from the measured behavior. These differences between the mathematical model and the structure occurred for a relatively simple structure where experimental measurements were available to improve the model. Therefore, it is not difficult to understand the need for adaptive control in LSS, where the structures are very large and complicated, and no experimental data will be available for improving the model before it is sent into space.

Adaptive controllers can be divided into two types, indirect and direct, depending upon whether or not the controller uses system identification. Adaptive controllers which apply system identification methods are referred to as indirect adaptive controllers, and generally couple the identification method with a controller which may range from classical pole placement to one of the robust method discussed above. For example, in ref. 13, Chiang and Cannon couple an LQG controller with an identification scheme called "Frequency Lock Loop" ,while in ref. 14, Sandararajan and colleagues use an identification process based on least-squares lattice filters coupled with a discrete time pole placement scheme. Both of these controllers were successfully implemented with good results at sampling rates of $25 \mathrm{~Hz}$ and $32 \mathrm{~Hz}$ respectively.

For identification based algorithms, the initial design of the controller is based on a rough model of the actual system. As the controller begins operation, the mathematical 
model of the system is updated by the system identification method, and the controller is then redesigned based upon the updated model. If the performance of the system meets design objectives, the process is complete, and the controller essentially becomes a nonadaptive controller until the system undergoes some change and system identification is required again. However, if the performance of the system does not meet the design objectives, the system identification process is started again, and the identification and redesign process is repeated until the desired performance is reached.

Two questions which identification schemes must answer are, first, will the identification scheme converge, and second, will it converges to the proper model of the system. In fact, in ref. 15 Kosut poses the following questions: "The fundamental question is: when will it work? That is, under what conditions will the model estimate converge to a good model in the allowed model set?" He further states: "By a good model is meant one that produces a controller, via the control design rule, which when applied to the actual system yields acceptable closed-loop performance. Even if such a good model exists, the estimated model may not converge, even if the estimated model is initialized close to a good model. Moreover, if convergence is too slow, then unacceptable behavior can occur during the learning process." In ref. 16, Kosut presents a method of designing a robust adaptive controller and established conditions under which the procedure will actually converge. Middleton, and colleagues (ref. 17) suggests that running parallel estimators is another way of overcoming the stabilizability problem of the identification process.

Assuming that the identification scheme can eventually produce an accurate model of the structure, the resulting model could be used in either the non-adaptive algorithms just described, or in conjunction with the second type of adaptive controllers frequently called direct adaptive controllers or self-tuning regulators. These direct adaptive algorithms update the controller gains directly and do not require system identification, although they would benefit greatly by having an accurate model from an identification scheme. Directs methods can also be sub-divided into two general types depending upon 
their method for updating the controller gains. The first type uses a switching condition to update the controller gains, and the second type uses model following techniques.

Examples of switching type adaptive controllers can be found in ref. 18 where Willems and Byrnes present an adaptive algorithm for a single-input single-output system and then extend it to multi-input multi-output systems in ref. 19. In simple terms, the controller switches back and forth between a series of gain matrices or functions, until it finds one or a combination which provides good performance. Direct adaptive controllers which use a switching condition to update the control gains have their beginning in ref. 20, where Morse suggested that when using a specified controller form, it was impossible to stabilize a general first-order dynamic system with an unknown parameter. In ref. 21 Nussbaum proves that Morse was correct for the controller form suggested. However, he introduced an alternate form for the controller which did stabilize a first order system. Morse then responded in ref. 22 by introducing a much simpler switching controller, and eased some of the stability requirements. However, to this point these papers focused more upon the existence of the controllers rather than a controller which could be implemented. Willems and Byrnes (refs. 18 and 19) were the first to be successful at moving the switching controller into the practical arena. Two challenges that faced the algorithm developed by Willems and Byrnes were the amount of prior knowledge required about the system and the inability of the designer to directly control critical performance parameters such as damping. In Contrast, direct adaptive controllers which use model following techniques have been in development since the late 1950's and Aström presents many industrial uses (ref. 3). These algorithms appear to be more developed than the switching algorithms, and allow the designer to specify the performance of the system, provide good stability margins, and are simple to implement.

\subsection{Model Reference Adaptive Control}

The model following adaptive algorithm used in this work is known as Model Reference Adaptive Control (MRAC) and the performance of the system is specified by 
some ideal reference model. In MRAC algorithms, the designer chooses an ideal mathematical model (the reference model) of the structure to be controlled, and the controller gains are continually updated to force the output of the actual system to follow the output of this ideal reference model. In this way, the designer can specify natural frequencies, damping ratios, and other important performance parameters. The idea of MRAC was first introduced in the late 1950's by Whitaker of the M.I.T. Instrumentation Laboratory, (refs. 23-24), and was based on minimization of the integral of the response error between the reference model and the actual system squared. This method of design has since been referred to as the M.I.T. rule. The M.I.T. rule was very popular because it could be implemented very easily in real-life applications. More recent MRAC algorithms have used Lyapunov stability analysis to design and guarantee stability (e.g., ref. 25). Typically in these methods, the adaptive update law is designed so that the first and second derivatives of the chosen Lyapunov function meet the necessary conditions of Lyapunov stability. Hang and Parks suggest that early algorithms designed with this method required that the entire state vector of the plant be measured (ref. 26), and Livner and Slater showed that problems with multi-input multi-output systems and unmodeled dynamics still existed (ref. 27). However, Broussard and O'Brien developed an algorithm for LSS which allowed the size of the reference model to be smaller than the actual system, and thus the number of required measured states has been reduced (ref. 28).

Many of the recent research efforts into MRAC can be traced back to a MRAC algorithm designed by Sobel and associates (ref. 29), in 1982. In a 1983 paper by BarKana and colleagues (ref. 30) it was shown that the conditions to guarantee stability could be relaxed from the conditions developed in ref. 29. Reference 30 further showed that, for a simply supported beam, if the sensors and actuators were colocated then the relaxed stability criteria were met. Ih et al. (ref. 31) suggest that this condition of colocated sensors and actuators will guarantee stability of all LSS with small but non-zero inherent damping. Bar-Kana and colleagues (ref. 30) also showed that if a combination of position and velocity data was used by the controller, the allowable percentage of positional 
interaction in the output was limited by a function dependent on the damping ratio and the lowest structural frequency. In ref. 32 Mufti takes the work of Bar-Kana et al. (ref. 30), further by showing that the amount of position-to-rate interaction is limited by twice the product of the damping ratio and the lowest structural frequency.

A group of researchers at the Jet Propulsion Laboratory (JPL) in Pasadena, CA have shown great interest in MRAC and have done some very useful work in the area. In ref. 33, Ih and associates address the issue of MRAC's lack of robustness to rigid body dynamics. They solve the problem by adding what they call an inner-loop plant augmentation, which essentially is a feedback loop around the plant. The end result is that the rigid body modes no longer have zero frequencies and the plant is stable. One additional change made to Sobel's initial algorithm by Ih and associates is the introduction of two gain weighting matrices which allow the designer to selectively increase the control effort applied to specified actuators. Measurement noise is addressed by Bayard and colleagues in ref. 34, and it is shown that without filters, the control forces becomes unrealistically high. The problem is solved by using a concept called a branch filter, which filters the output error between the reference model and the plant without introducing phase lag into the adaptive loop.

Building upon the results of refs. 33 and 34, Ih and associates tested MRAC experimentally (ref. 31) on an antenna-like structure (12 feet in diameter) built at JPL. A set of proof-of-concept adaptive control experiments for transient and initial deflection regulation were conducted. Results show that the regulation capabilities of MRAC are excellent, and the MRAC algorithm can reduce maximum overshoot and settling time. Results from more complex experiments were presented by Ih et al. in ref. 35, where six input/output pairs were used for multi-variable adaptive control. Practical implementation issues like computational requirements and lack of velocity sensors were addressed and overcome. In Ref. 36 Wittenmark and Aström suggested that MRAC was not robust to unmodeled dynamics in some situations, but the results of the JPL experimental work indicate that for the JPL antenna-structure, unmodeled dynamics were not an important 
factor.

In ref. 37 Ih et al. present results of more experiments, where robustness of MRAC to input saturations and time delay effects were studied. It is shown experimentally that for the JPL structure, the JPL MRAC algorithm is robust to input saturation, but the effect of time delays can significantly degrade the performance and even stability of the algorithm. However, it is also shown that using the technique advocated by Bar-Kana and Kaufman (ref. 38), of placing a feed-forward loop on the plant, stabilizes the closed-loop system and recovers some of the performance. The most recent experimental result presented by the JPL researchers are found in ref. 39. In this latest work, the issues of performance of MRAC for pointing and tracking control are studied. As with their other experimental work, MRAC performed very well in these experiments.

Throughout all the previous work two questions have remained unanswered. First and foremost is the question of control cost to implement MRAC. By definition, the need for adaptive control arises due to a lack of an accurate model for the actual structure. Therefore, faced with this lack of knowledge, how does one know what to use for a reference model, or for that matter, does it make any difference what reference model is chosen? It seems logical to assume that if the reference model is very close to the actual structure, MRAC will be very effective, and the control cost will be minimal. However, the question of what happens to the control costs if the reference model is considerably different than the actual structure is an important question. If control costs remain constant for all reference models, then MRAC would be an ideal controller. However, if a small difference between the reference model and the plant produce large increases in the control costs, then great care must be taken to somehow select a reference model close to the actual plant.

A second question that has gone unanswered until just recently deals with the actual design of the MRAC controllers. The MRAC algorithms of refs. 29-34 all require the designer to specify the elements of several potentially large matrices, and no rule for choosing these matrices is available. In two papers (refs. 40 and 41) Bayard makes a first 
attempt at finding a systematic method for choosing elements of these matrices. Specifically, he approaches the problem from an optimization point of view to see if the weighting matrices can be chosen to optimize some measure of performance and minimize control cost.

\subsection{Objectives}

The objectives of this work are to answer the two questions discussed above. Specifically, while it is possible to design an adaptive controller based on a poor model of a structure and still achieve stability and performance (e.g. refs. 31, 35, 37, 39), the required control costs may be very high. The first objective of this work is to understand how differences between the plant and the reference model affect the control cost. The second objective is to consider the question of choosing the weighting matrices to maximize the performance of MRAC while minimizing the control cost. However, the optimal designs will be considered useful only if experimental results show the same improvement trends. Since most of the research into adaptive controllers has been strictly theoretical in nature and experimental verification of the proposed theories is lagging behind, (compare refs. 1 and 2 with 3 and 4), the final objective is to compare analytical calculations with experiments to verify every result.

\subsection{Approach}

In this work MRAC is applied to two structural systems which have high fidelity models, and intentional differences between the reference model and the plant are introduced. Control effort indices are monitored to determine the effects of small differences between the structure and the reference model on the required control effort. It is shown numerically and verified experimentally that reasonable amounts of difference between the structure and the reference model can cause dramatic increases in the control effort. Finally, optimization is applied to the controller weighting matrices and optimal 
designs are tested experimentally to see if the analytical performance and control effort gains can be realized. It is also shown that the weighting matrices can be optimized only up to a certain point after which the analytical optimal design shows large improvements, but the experimental results remain constant.

Chapter 2 introduces the MRAC algorithm, the performance indices monitored throughout the work, and the experimental and analytical implementation of MRAC. Chapter 3 describes the structures used, and discusses the choice of reference models for these structures. The experimental set up is outlined and the hardware components are discussed. Chapter 4 examines the effects of the various reference models on the control effort, and gives both simulation and experimental results. Chapter 5 defines the optimization problem and shows how the control effort can be reduced while still maintaining performance, and concluding remarks are made in chapter 6. 


\section{Chapter 2}

\section{Model Reference Adaptive Control}

MRAC is one of the more popular adaptive methods (refs. 29, 30, 32, 33, 34, 40) which does not require system identification. MRAC methods adaptively tune the controller gains, forcing the actual system to follow an ideal reference model. Because this reference model can be of lower order than a typical model of the actual system, this method is very attractive for application to LSS, where structural models can be of very high order and require truncation for use with practical controllers. Figure 2-1 shows a generalized block diagram of a MRAC system (ref. 30).

The structure to be controlled can be represented mathematically in standard state space form

$$
\begin{gathered}
\dot{X}_{p}(t)=A_{P} X_{p}(t)+B_{p} U_{p}(t), \\
Y_{p}(t)=C_{p} X_{p}(t),
\end{gathered}
$$

where $X_{p} \in R^{N p}, U_{p} \in R^{M}, Y_{p} \in R^{M}, A_{p}, B_{p}$, and $C_{p}$ are of appropriate dimensions, and the subscript $p$ denotes the plant. The term plant, as used throughout this text, refers to the actual structure (experimental), or the mathematical model (simulations) of the structure. It is assumed that $\left(A_{p}, B_{p}\right)$ is controllable, $\left(A_{p}, C_{p}\right)$ is observable, and that the number of inputs, $M$, is equal to the number of outputs. A stable reference model which specifies the desired performance of the structure is also described by a state space representation 


$$
\begin{gathered}
\dot{X}_{m}=A_{m r} X_{m}(t)+B_{m} U_{m}(t), \\
Y_{m}=C_{m} X_{m}(t) .
\end{gathered}
$$

where $X_{m} \in R^{N m}, U_{m} \in R^{M}, Y_{m} \in R^{M}, A_{m}, B_{m}$, and $C_{m}$ are of appropriate dimensions, and the subscript $m$ denotes the ideal reference model. As stated above, $N_{m} \ll N_{p}$ for LSS. It is also assumed that the plant and the reference model satisfy the command generator tracker (CGT) conditions (ref. 39). The CGT condition requires that the plant $\left(A_{p}, B_{p}, C_{p}\right)$ have no transmission zeros at the origin or in common with any eigenvalues of $A_{m}$. The function of MRAC is to force the output of the plant to follow the output of the reference model. How well the plant output follows the reference model is measured by an error vector $e_{y}$, defined as the difference between the measurement vectors

$$
e_{y}=Y_{m}(t)-Y_{p}(t) \text {. }
$$

Therefore, the objective of MRAC is to force $e_{y}$ to zero asymptotically.

\subsection{MRAC Algorithm}

The MRAC algorithm used here is based on the work of Sobel, Kaufman and Mabius (ref. 29), and is briefly summarized here. The control input is written as,

$$
U_{p}(t)=K(t) I(t)
$$

where

$$
I^{T}(t)=\left[\begin{array}{llll}
e_{y}^{T} & X_{m}{ }^{T} & U_{m}{ }^{T}
\end{array}\right],
$$

and the gain matrix $\mathrm{K}$ is composed of a proportional and an integral component

$$
K(t)=K_{p I}(t)+K_{I}(t),
$$


The adaptive laws for the gains $\mathrm{K}_{\mathrm{pr}}(\mathrm{t})$ and $\mathrm{K}_{\mathrm{I}}(\mathrm{t})$ are given as

$$
K_{p r}(t)=e_{y} I^{T} T^{*},
$$

and

$$
\dot{K}_{I}(t)=e_{y} I^{T_{T}},
$$

where $\mathrm{T}^{*}$ and $\mathrm{T}$ are time invariant weighting matrices of appropriate dimension chosen by the designer. As the magnitude of $\mathrm{T}$ and $\mathrm{T}^{*}$ increases, the plant follows the reference model more closely, but the control effort is also increased. However, Bayard has shown in ref. 40 , that there is a threshold point where increasing $T$ no longer improves the performance of the system. Thus, it is desirable to operate at or below this threshold. Sufficient conditions for global stability are summarized below (ref. 39).

1) The CGT condition is satisfied

2) $T$ and $T^{*}$ are positive definite and positive semi-definite respectively

3) The open-loop plant transfer function matrix is strictly positive real (see ref. 42 for a definition of strictly positive real).

According to Ih and associates (ref. 37), for a structure with small but non-zero inherent damping, no rigid body modes, and colocated sensors and actuators, the above conditions can be met by choosing $T$ and $T^{*}$ to satisfy condition 2 , and limiting the amount of displacement output used in eq. 2-5. Since the choice of $T, T^{*}$, and the amount of displacement output used in eq. 2-5 are up to the designer, and all structures have nonzero inherent damping, the only important requirement for stability of a structure without rigid body modes is colocated sensors and actuators. The structures and reference models used in this work use only velocity output and meet the above conditions so it is not 
necessary to apply the conditions to the structures separately, and they are included here only for completeness.

\subsection{Control Cost}

One of the most distinguishing and desirable characteristics of MRAC is its ability to force the plant to follow the reference model even when large differences between the plant and the reference model exist. Differences between the plant and reference model can occur because of errors in frequencies, mode shapes, and damping ratios. Differences also occur because the order of the reference model is less than the plant. In this work, we consider only differences in frequency, mode shapes and damping ratios. The effects of these differences on the control effort are quantified by two parameters:

(1) the maximum control force required by each actuator (1 to $M$ ),

$$
\begin{gathered}
U_{\max }^{i}=\operatorname{Max}\left(\left|U^{i}(t)\right|\right) \\
0 \leq t \leq t_{\text {final }} \\
i=1, \ldots, M
\end{gathered}
$$

(2) the control effort (CE),

$$
C E=\sqrt{\int U^{T} U d t}
$$

order to find the effect on these two parameters of the amount of difference between the plant and reference model, the following procedure has been adopted. First, an accurate model is developed which represents an actual structure. Next, a reference model is chosen which specifies the desired performance of the system and has some measurable amount of error between it and the plant. Previous researchers (ref. 30) have chosen the reference model to be a reduced order model of the plant with exact frequencies and mode shapes but additional damping. While it would be ideal if MRAC designers had 
this exact knowledge of the plant and could choose the reference model to be a reduced model with exact frequencies, it is not probable. In order to model this difference between the plant and reference model, we have intentionally introduced differences between the reference model and the plant.

\subsection{Integration Algorithm}

Because the analog control scheme of eq.'s $2-5$ to $2-10$ is implemented on a digital computer, great care must be taken in the integration algorithm to model the transfer of force and velocity information between the structure and the digital controller. The control loop can be broken down into three distinct step. The first step is the measurement step where the instantaneous velocities of the structure are measured, and made available to the integration algorithm. In the second step, the digital computer uses the velocities from step 1 to calculates the control force to be applied to the structure, and calculates the states of the reference model at a time $\Delta t$ in the future, where $\Delta t$ is the time step between successive velocity measurements. The final step is to output the control forces calculated by the computer. Because of the digital nature of the computer the structure see a series of step inputs of duration $\Delta t$, and the integration algorithm sees a series of step velocities.

Equation 2-10 is a set of differential equations of order $M \times\left(2 M+N_{m}\right)$. Therefore, the total number of equations which the controller must integrate in real time is $\mathbf{M ~} \mathbf{x}$ $\left(2 \mathrm{M}+\mathrm{N}_{\mathrm{m}}\right)+\mathrm{N}_{\mathrm{m}}$ (plant equations are not included because the structure integrates itself). Since the number of equations can become large even for relatively small models, (for example, a 6th order reference model with $M=3$, gives 42 equations), an integration scheme with both speed and accuracy was required. A simple Euler explicit technique and a Runge Kutta algorithm were found to be inaccurate and too slow, respectively. A 
3rd order Euler implicit scheme with Newton iteration was selected for achieving good accuracy and high speed.

\subsubsection{Experimental Implementation}

The only quantities passed between the structure and the controller are the measured velocities and the control forces applied to the structure. To implement this process the reference model equations, (2-3 \& 2-4), and the integral gain equations, (210), are integrated as follows. Based on the states of the reference model, the integral gains, and the measurements from the structure at a time $\left(\mathrm{t}=\mathrm{t}_{0}\right)$, the following equations are evaluated:

$$
\begin{gathered}
e_{y}\left[t_{0}\right]=Y_{m}\left[t_{0}\right]-Y_{p}\left[t_{0}\right], \\
I^{T}\left[t_{0}\right]=\left[e_{y}\left[t_{0}\right] X_{m}\left[t_{0}\right] U_{m}\left[t_{0}\right]\right], \\
K_{p I}\left[t_{0}\right]=e_{y}\left[t_{0}\right] I^{t}\left[t_{0}\right] T^{*}, \\
K\left[t_{0}\right]=K_{p r}\left[t_{0}\right]+K_{I}\left[t_{0}\right] .
\end{gathered}
$$

Using the above results, the control force is calculated as

$$
U_{p}\left[t_{0}\right]=K\left[t_{0}\right] r\left[t_{0}\right] .
$$

and sent to the structure. While the controller waits for the next velocity measurement at $t=t_{0}+\Delta t$, the results of equation $2-13$ are used to calculate the states of the reference model and the integral gains at $t=t_{0}+\Delta t$ as follows. Writing equations $2-3,2-4$ and 2-10 as

$$
\dot{x}(t)=f(X),
$$


where $\mathrm{X}$ is made up of the reference model states, and the integral gain states. Using a Taylor series expansion and applying Newton's iteration, the final equation (see Appendix A) for calculating the change in the states $(\Delta X)$ is:

$$
\left[\frac{\Delta t}{2} \frac{\partial f}{\partial X}-I\right] \Delta X=\left[X^{n+1}-X^{n}-\frac{\Delta t}{2}\left(f^{n+1}+f^{n}\right)\right]
$$

where $I$ is the identity matrix, $X^{n}$ is the known states at $t=t_{0}, X^{n+1}$ is an estimate of the states at $t=t_{0}+\Delta t$ and $f^{n+1}=f\left(X^{n+1}\right)$, and $\partial f / \partial X$ is the Jacobian matrix,

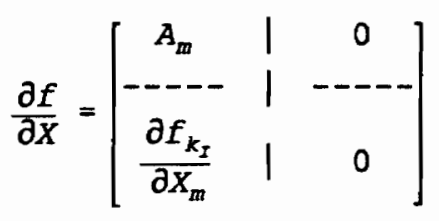

A full derivation of this equation can be found in Appendix A. Equation 2-19 is a linear system which must be solved at each iteration. If we set

$$
\left[\begin{array}{l}
Q_{m} \\
Q_{K I}
\end{array}\right]=\left[X^{n+1}-X^{n}-\frac{\Delta t}{2}\left(f^{n+1}+f^{n}\right)\right],
$$

where subscript $m$ refers to reference model equations and subscript $\mathrm{KI}$ refers to integral gain equations, then equation 2-19 takes the form

$$
\left[\begin{array}{lll}
\frac{\Delta t}{2} A_{m}-I & 1 & 0 \\
\hdashline \frac{\partial f_{K I}}{\partial X_{m}} \frac{\Delta t}{2} & 1 & -I
\end{array}\right]\left[\begin{array}{l}
\Delta X_{m} \\
-\overline{\Delta X_{K I}}
\end{array}\right]=\left[\begin{array}{c}
Q_{m} \\
-Q_{K I}
\end{array}\right] \text {, }
$$

which can be separated into the set of equations 


$$
\begin{gathered}
\left(\frac{\Delta t}{2} A_{m}-I\right) \Delta X_{m}=Q_{m I} \\
\left(\frac{\partial f_{K I}}{\partial X_{m}} \frac{\Delta t}{2}\right) \Delta X_{m}-\Delta X_{K I}=Q_{K I} .
\end{gathered}
$$

Solving equation 2-23 for $\Delta X_{m}$ as

$$
\Delta X_{m}=\left[\frac{\Delta t}{2} A_{m}-I\right]^{-1} Q_{m}
$$

and substituting $\Delta X_{m}$ into equation $2-24$, the solution for $\Delta X_{K I}$ is

$$
\Delta X_{K I}=\frac{\Delta t}{2} \frac{\partial f_{K I}}{\partial X_{m}} \Delta X_{m}-Q_{K I}
$$

If $A_{m}$ is chosen to be independent of time, the matrix inverse in Equation 2-25 is calculated only once. The initial estimate for $X^{n+1}$ is $X^{n}$, and during each iteration the value of $\mathrm{X}^{\mathrm{n}+1}$ is updated as,

$$
X^{n+1}=X^{n+1}+\Delta X
$$

and convergence is reached when,

$$
i=1, \ldots, M\left(2 M+N_{m}\right)+N_{m}
$$

where $\varepsilon$ is the desired accuracy. After convergence is reached, the algorithm waits for the next measurement and the process begins again.

\subsubsection{Simulation Algorithm}

In order to simulate the transfer of information between the controller and the physical system, the simulation algorithm integrates two separate systems of equations. 
In addition to the equations of the previous section, the equations of the structure (plant) must also be simulated. Integrating these two systems of equations separately was the key to maintaining the integrity of the force and velocity information exchanged between the controller and structure. The following process was followed. At $t=t_{0}$ the velocity states of the reference model and plant are stored, and used to calculate the control forces as in equations 2-13 through 2-17. The error vector $e_{y}$ is then used in the iterative equations 2-19 through 2-27 to calculate the new states of the reference model at $t=t_{0}+\Delta t$. Finally, the control force $U_{p}\left[t_{0}\right]$ and appropriate iterative equations (2-19 through 2-27) are applied to the equations of the plant to find the updated states of the plant. Note that when $\mathrm{X}^{\mathrm{n}+1}$ is updated and $\mathrm{f}^{\mathrm{n}+1}$ is recalculated in the iterative process, the plant velocities are kept fixed in the solution for the reference model, and the control force is fixed at $\mathrm{U}_{\mathrm{p}}\left(\mathrm{t}_{0}\right)$ in the iterative process for the plant. In this way the digital effects of the controller on the actual system is simulated.

MRAC (equations 2-3 through 2-10) can force the plant to follow the reference model even when large differences between the plant and the reference model exist. This work intentionally introduces known differences between the plant and the reference model (frequencies, mode shapes, and damping ratio errors), and measures maximum control force and control effort (equations 2-11 \& 2-12) to monitor the effects of these differences. In both the Experimental and analytical implementation, integration of the reference model equations is done using a 3rd order Euler scheme with Newton iteration, and the form of the Jacobian matrix (equation 2-20) allows for a simplified solution (equations $2-25$ \& 2-26). Although the analytical simulation must also integrate the equations of the actual structure, special care is taken to simulate the measured velocity and the digital effects of the controller on the actual system. 


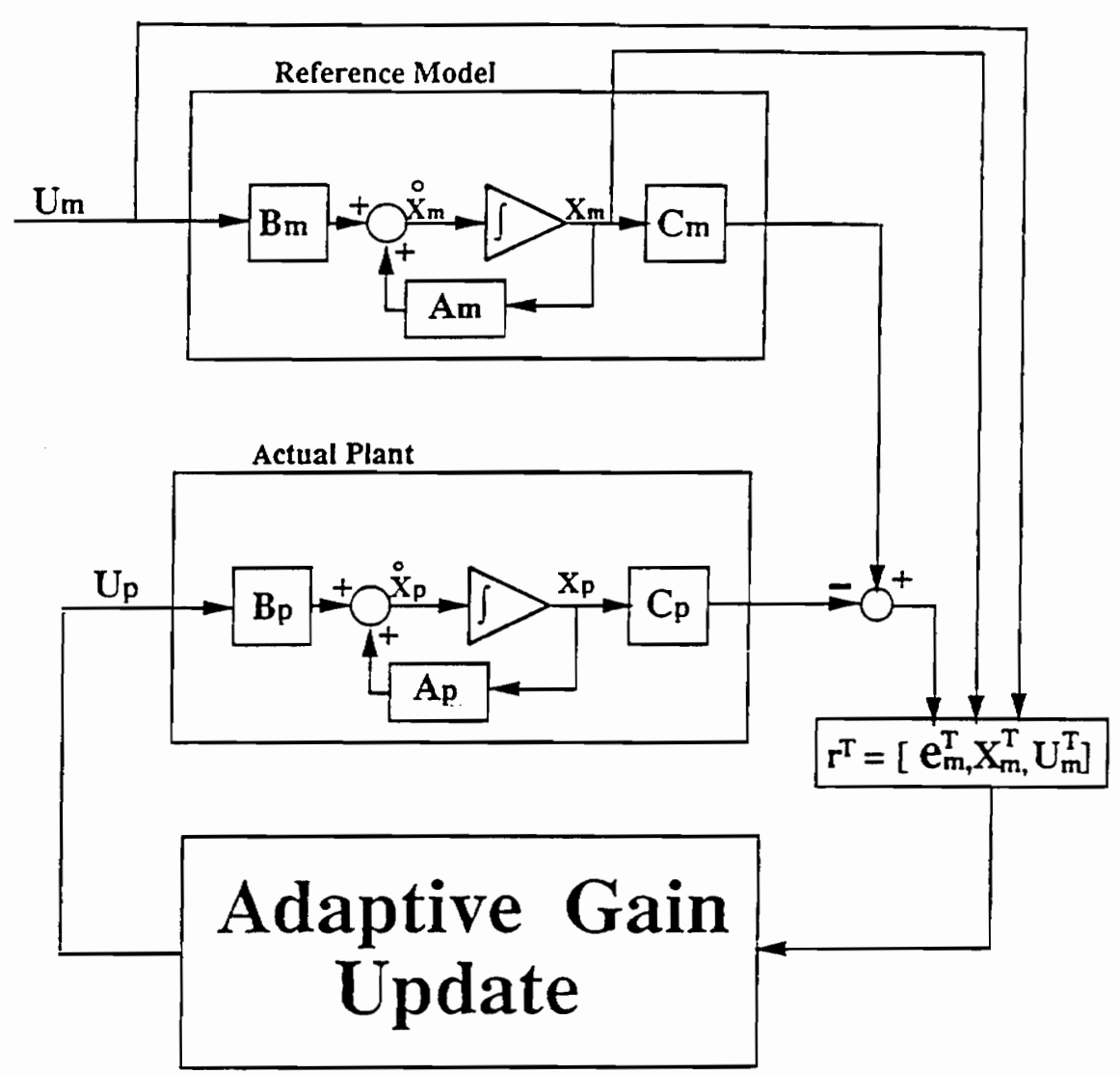

Figure 2-1

Model Reference Adaptive Control Block Diagram 


\section{Chapter 3}

\section{Example Structures: Analytical Models and Experimental Set Up}

In this chapter, the two laboratory structures used in the work, the reference models which specify the ideal performance, and the hardware used in the experimental set-up are introduced and discussed. The Suspended Mass system is discussed first, followed by the Slewing Grid. A brief description of their physical characteristics is given followed by a discussion of modeling techniques. Next, the reference models for the two systems are discussed, followed by a description of the sensors, actuators, and other hardware used in the experimental set up.

\subsection{Structural Models}

In order to evaluate MRAC experimentally and analytically, both laboratory structures and mathematical models which simulate the frequency and damping characteristics of the structures were required. Ideally, the laboratory structures would exhibit many of the characteristics of proposed LSS: low frequency modes, high modal density, low inherent damping, and rigid body modes. Of the two structures used in this study only one was designed to have the above mentioned characteristics, the other was designed for simplicity and accuracy. The Suspended Mass is a structure designed to be both simple and easy to accurately model analytically as a Single Degree Of Freedom (SDOF) system, while the Slewing Grid was designed to have characteristics of proposed LSS. Together, the two structural systems allow us to start with a simple and accurate analytical model, and then move to a more complex structure with a less accurate model. 


\subsubsection{Suspended Mass System}

The Suspended Mass System shown in figure 3-1 is made of a mass suspended by four equal length wires. The cylindrical stainless steel mass is 1.0 inches thick, 3.8 inches in diameter, and weighs $3.375 \mathrm{lbs}$ (including attached actuator/sensor coils). The mass is held in place and connected to the supporting structure by four 0.025 inch diameter wires. The wires are connected to the mass and the supporting structure with $3 / 16$ inch eye bolts which are used to adjust the tension in the wires. The base of the support structure is an aluminum plate ( $24 \times 24 \times 3 / 8$ in.) which is connected to the wires by four right angle steel connectors. Motion of the mass is perpendicular to the base and velocity is measured by a single velocity sensor attached to the top of the mass. In a similar manner, control forces are applied by a colocated force actuator attached to the bottom of the mass.

The analytical model of this system is the standard spring-mass-damper system (figure 3-2), where the spring constant $k$ is proportional to the tension $\left(T_{A}\right)$ and the inverse of the length $(L)$ of each wire $\left(k=4 T_{A} / L\right)$, and the damping is provided by the inherent structural damping in the wires, air resistance, and friction. Figure 3-3 shows a magnitude and phase plot of a velocity frequency response for the Suspended Mass System, where volts in represents the magnitude of the forcing function and volts out represents the magnitude of the peak velocity due to the force input. The single peak and 180 degree phase change indicate an excellent lightly damped SDOF system. The equation of motion for the system is

$$
m_{p} \dot{x}_{p}(t)+c_{p} \dot{x}_{p}(t)+k_{p} x_{p}(t)=u_{p}(t),
$$

where $m_{p}, c_{p}, k_{p}$, and $u_{p}$ are scalar quantities representing the mass, damping coefficient, stiffness, and control force of the plant respectively. The state space representation is

$$
\left[\begin{array}{l}
\dot{x}_{1 p} \\
\dot{x}_{2 p}
\end{array}\right]=\left[\begin{array}{cc}
-2 \zeta_{p} \omega_{n p} & -\omega^{2}{ }_{n p} \\
1.0 & 0
\end{array}\left[\begin{array}{l}
x_{1 p} \\
x_{2 p}
\end{array}\right]+\left[\begin{array}{c}
1 \\
m_{p} \\
0
\end{array}\right] u_{p}(t),\right.
$$


where $x_{1 p}$ and $x_{2 p}$ are the velocity and displacement of the mass respectively, $\omega_{n p}$ is the measured natural frequency, and $\zeta_{p}$ is the measured damping (because $\zeta_{p}$ is so small, the assumption that $\omega_{n p}=$ the measured natural frequency, is correct to six decimal places).

To accurately measure the natural frequency of the Suspended Mass System, a pseudo sine sweep procedure was used. The experimental velocity trace of the mass was compared with the analytical velocity trace. The frequency of the analytical system was varied until the two traces matched very closely for 2 seconds. Figure 3-4 shows two such runs where the frequency difference between analytical and experimental traces in the top figure is $0.1 \mathrm{~Hz}$, and the difference in the bottom figure is close to zero. Due to changes in the tension of the wires that hold the mass in place (temperature changes, creep, and slipping of connections), the natural frequency $\left(\omega_{n p}\right)$ of the system changes over time. When the system was initially assembled, $\omega_{\mathrm{up}}$ was $6.8 \mathrm{~Hz}$. However, at the present time, the frequency is around $6.0 \mathrm{~Hz}$. The damping of the system was determined by linear regression. It was necessary to use linear regression because the small value of inherent damping and small inconsistencies in peak velocities cause simpler methods to vary radically from one measurement to the next. The equation of the decay envelope is assumed to be

$$
\operatorname{Max}\left(x_{2 p}\right)=A e^{-\zeta_{p} \omega_{n p} t},
$$

where $\mathrm{A}$ is a constant, $\zeta_{\mathrm{p}}$ is the damping ratio, and $\mathrm{t}$ is the time at which the peak values of $\mathrm{X}_{2 \mathrm{p}}$ (velocity) occur. Taking the natural logarithm of both sides yields

$$
\log \left(\operatorname{Max}\left(x_{2 p}\right)\right)=\log (A)-\zeta_{p} \omega_{n p} t .
$$

Equation 3-4 is the equation of a straight line, and linear regression is used to solve for the slope of the line and thus $\zeta_{p}$. Figure 3-4 shows a typical free response used to get damping data, and also indicates how small the inherent damping is. Using the above procedure, the damping ratio $\zeta_{p}$ was calculated to be 0.00067 . 


\subsubsection{Slewing Grid Structure}

The Virginia Tech slewing grid laboratory structure, shown in figure 3-5, is a more complex example. The slewing grid was designed to have characteristics of LSS, namely, closely spaced modes, low natural frequencies, and low inherent damping. Three pairs of velocity sensors and force actuators (see section 3.3) are colocated at joints 3, 4, and 5. Although the geometry of the structure is symmetric about a horizontal line through joint 3 , the vibration mode shapes are not similarly symmetric, because the structure's weight causes asymmetric member gravity loading and therefore an asymmetric stiffness distribution. Due to gravity loading, the lower horizontal member is compressed to about $70 \%$ of its Euler (pin-ended) buckling load.

Great effort has been taken to accurately predict the loads in each member of the structure. However, each joint is held in place with a nut and bolt assembly and the process of tightening these bolts induces forces into the members, and these forces have not been accurately determined. The current Finite Element Model (FEM) only takes gravity forces into account. The rotational shaft was modeled with 8 finite elements with displacement and rotation Degrees Of Freedom (DOF) at each node. Each of the 5 members of the structure is modeled with 4 finite elements which include a transverse displacement, an in-plane rotation, and an out-of-plane rotation at each node. The complete FEM has 72 DOF, and the coupled equations of motion can be written as

$$
M \ddot{X}+C \dot{X}+(K+G) X=F=b_{c} U,
$$

where $G$ is the geometric stiffness matrix and $b_{c}$ is a matrix with only three non-zero terms used to direct the control forces $U_{i}$ to the proper DOF. To make the problem more manageable, a reduced eleventh order model was created using the Guyan reduction (ref. 43). However, it should also be noted that even with reduced order mass, stiffness, and damping matrices (11 DOF), and only 3 inputs/outputs, simulating MRAC applied to the Slewing Grid still required integrating 128 equations. If MRAC were implemented on the Slewing Grid, the plant equations would not need to be integrated by the controller, 
because plant velocities would be measured not calculated, but that only reduces the size by 22 equations, still leaving 106 equations which would need to be integrated in real time. The linear equations for the Slewing Grid can be written (in physical coordinates) as,

$$
\begin{gathered}
{\left[\begin{array}{c}
\dot{X} \\
\dot{X}
\end{array}\right]=\left[\begin{array}{cc}
-M_{I}^{-1} C_{x} & -M_{I}^{-1} \hat{K}_{r} \\
I & 0
\end{array}\right]\left[\begin{array}{l}
\dot{X} \\
X
\end{array}\right]+\left[\begin{array}{c}
M_{x}^{-1} b_{c} \\
0
\end{array}\right] U,} \\
Y=\left[\begin{array}{ll}
B^{T} & 0
\end{array}\right]\left[\begin{array}{l}
\dot{X} \\
X
\end{array}\right],
\end{gathered}
$$

where

$$
\hat{K}_{I}=K_{x}+G_{x}
$$

and $M_{r}, C_{r}, K_{r}$, and $G_{r}$ are the reduced mass, damping, stiffness and geometric stiffness matrices, and $B$ is a ( $22 \times 3)$ matrix with only 3 non-zero elements for mapping the three control inputs to the proper DOF at joints 3,4, and 5.

The accuracy of the frequencies and mode shapes predicted by the FEM is not good. Over a two year period, much effort was devoted to developing a FEM which would accurately model the structure. However, the non-linearities in the structure, such as friction in the bearings, large gravity loading in the lower horizontal member, and the loads induced by tightening the bolts at each joint, have been difficult to model. Table 3-1 compares frequencies predicted by our best FEM to the experimental vibration frequencies, and figures 3-6 through 3-12 compare several experimental and analytical mode shapes. Appendix B gives a more detailed description of the modeling process and the efforts to get the model to match the measured results. The challenges of modeling the slewing grid may be similar to those faced when modeling LSS except that with many LSS we will not have experimental data available for improving analytical models. 


\subsection{Choosing the Reference Model}

In order to study the variation of control effort and maximum force for various amounts of difference between the reference model and the plant, the reference model must have two characteristics. First, the reference model must accurately model the plant, and second, there must be some means of varying a parameter in the reference model to produce controlled amounts of difference between the two models. In this way we can find the control effort and maximum force required for the perfect model case and see how the two cost parameters change as a function of the differences between the two models.

\subsubsection{Suspended Mass Reference Model}

The form of the state space representation of the reference model is similar to the actual system except the control force $U_{m}$ is set to zero because we wanted the reference model to be a damped free response,

$$
\left[\begin{array}{l}
\dot{x}_{1 m} \\
\dot{x}_{2 m}
\end{array}\right]=\left[\begin{array}{cc}
-2 \zeta_{m} \omega_{n m} & -\omega_{n m}^{2} \\
1.0 & 0.0
\end{array}\right]\left[\begin{array}{l}
x_{1 m} \\
x_{2 m}
\end{array}\right],
$$

where the subscript $\mathrm{m}$ refers to the reference model. Since the system is almost completely described by its natural frequency, differences between the two systems are introduced by varying the natural frequency of the reference model by $\pm 50 \%$ in $10 \%$ increments.

$$
\begin{gathered}
\omega_{n m}=\alpha\left(\omega_{n p}\right) \\
0.5 \leq \alpha \leq 1.5,
\end{gathered}
$$

where $\alpha$ is a measure of the difference between the natural frequencies of the reference model and the actual system. In addition to the natural frequency difference of the reference model, the reference model has $10 \%$ damping $\left(\zeta_{m}=0.1\right)$ while the actual system only has $0.067 \%$ damping. The damping of the reference model is typical of the 
requirements for LSS.

\subsubsection{Slewing Grid Reference Model}

In order to study the performance of MRAC for this case we first had to choose an accurate model for simulating the laboratory structure. Our efforts to model the slewing grid as accurately as possible resulted in several FEM's with varying degrees of accuracy. The most accurate model used experimental frequencies and mode shapes in a model correction method, proposed by Baruch and Bar-Itzhak (refs. 44-45), to force the theoretical model to have the exact experimental frequencies. Appendix $\mathrm{C}$ presents a complete discussion of the model reduction and correction process. The least accurate model was the standard FEM with no corrections. When applying MRAC to the slewing grid, error is introduced into the reference model by taking a linear combination of the standard FEM and the corrected FEM, while leaving the desired damping constant,

$$
\text { Ref. Model }=(1-\alpha)(\text { standard FEM })+\alpha(\text { corrected FEM }) \text {, }
$$

where $\alpha=1$ represents the perfect modeling case, and $\alpha=0$ is the case of maximum error. Five different reference models were tried with $\alpha$ taking the values $0.0,0.25,0.5$, $0.75,1.0$. Damping ratios for the Slewing Grid were obtained experimentally, while the damping ratios for the reference model specify the desired performance (see table 3-2).

\subsection{Sensors and Force Actuators}

Two types of non-contacting sensors were used in this work. The first, a displacement probe produced by Bently-Nevada (figure 3-13), was used to collect vibration data used in the modal analysis and in developing the experimental mode shapes shown in figures 3-6 through 3-12. It was also used in the control experiments to provide a precise initial displacement and instantaneous displacement readings to the controller. The probe is mounted on a magnetic base to the supporting structure and moved to within approximately 0.07 inches of a small stainless steel target. The output of the probe is a 
negative voltage with the linear range -1 to -11 volts. The sensitivity of the probe is approximately 100 volts per inch, and the probe is usually set in the static position at -6 volts. As the gap between the probe tip and the target changes, the output voltage changes, allowing displacements of \pm 0.05 inches to be measure with \pm 0.0001 inch accuracy.

The second type of sensor used is a velocity sensor which consists of a structureborne coil which is placed in an annular magnetic field housing attached to the support structure (figure 3-14). Movement of the conducting coil inside the magnetic field causes a voltage proportional to the velocity of the coil. The coil leads must be connected to a high input impedance to prevent back EMF that would distort the signal and add damping to the structure. Three problems make these velocity sensors somewhat difficult to use. First, the wire on the coil is unshielded and can act like an antenna for any $60 \mathrm{~Hz}$ noise in the area. Second, the linear range of these coils is very small, and third, the dynamic calibration can be $10 \%$ to $20 \%$ different than the static calibration. Appendix D gives a complete description of the calibration procedures and results.

The force actuators used are based on the same principle as the velocity sensors. In fact, the coil/magnetic field assembly can be used for either purpose. To produce a control force on the structure, a voltage proportional to the required force is sent from the controller to a power amplifier which outputs a proportional current. This current is applied to the coil, and since a magnetic field exerts a sideways force on a moving charge (ref. 46), a force parallel to the center line of the coil is produced (see figure 3-15). The current is controlled so that the fluctuating voltage produced by the motion of the coil does not affect the force applied to the structure (ref. 12).

\subsection{Controller}

As stated above, implementation of MRAC can become very computationally expensive even for a low-order reference model, (a FEM with 11 DOF and three input/output pairs requires that 84 differential equations be integrated in real time). 
Therefore the controller must have hardware capable of the necessary speed, and the software must use the hardware efficiently. The controller used in this work was a TMS320C30 System Board manufactured by Spectrum Signal Processing, Inc. The board plugs into a standard IBM-AT and is accessed through a driver program written by the

user. The System Board provides two synchronized input/output channels with 16-bit A/D and D/A converters, and a TMS320C30 (C30) Digital Signal Processor (DSP) manufactured by Texas Instruments. The C30 is a high-performance CMOS 32-bit floating-point device in the TMS320 family of single-chip digital signal processors (ref 47). The C30 is a high-speed, DSP with a 60-ns cycle time which allows it to execute operations at a performance rate up to 33 million floating-point operations per second. The System Board has a programmable timing circuit which allows the user to execute control algorithms at rates up to $151.5 \mathrm{kHz}(6.6 \mu \mathrm{sec} / \mathrm{sample}$ time cycle) (ref. 48). Programming the $\mathrm{C} 30$ can be done in several languages, but $\mathrm{C}$ and assembly were used exclusively in this work. $C$ is the easiest way to program the $C 30$ but when maximum performance is required, assembly language must be used. The driver program written and run on the IBM-AT processor communicates with the $\mathrm{C} 30$ allowing the programmer to start and stop the controller as well as pass critical information back and forth between the two processors. Although $\mathrm{C}$ is a high-level language, programming the $\mathrm{C} 30$ requires system level tasks and is very tedious and detail oriented.

\subsection{Experimental Set Up and Procedure}

The experimental setup shown in figures 3-16 to 3-19 connects the Suspended Mass to the controller through a series of elementary electrical circuits. A test begins by giving the mass a known set of initial conditions. A -3 volt output signal from channel B of the DSP board causes the switching circuit (figures 3-16 and 3-19) to be closed, allowing for a DC voltage from the function generator to reach the summing circuit. The second input of the summing circuit comes from channel A of the DSP board, and is set to zero. Thus the output of the summing circuit causes an initial displacement. When the initial 
displacement has been set and the velocity of the mass is zero, channel B sends a +3 volt signal which opens the switching circuit, cutting the D.C. voltage off, and the mass begins to vibrate. However, when the current is switched off, the force applied to the mass does not go to zero instantaneously, but has a transient response (figure 3-20) which takes about $300 \mu \mathrm{sec}$ to decay. This transient response is caused by residual currents generated as the inductance built up in the coil tries to settle back to zero. This fluctuation of current has the effect of applying several impulses to the system. To avoid as much as possible the transient behavior due to these impulses, the system is allowed to vibrate freely for approximately two seconds, allowing these unmodeled high frequency transients to die out. After this initial settling period, the instantaneous velocity and displacement of the mass are sampled and sent through the appropriate circuits (figs. 3-17 and 3-18) to the DSP where the reference model is waiting for initial conditions. In this way the reference model starts with the same initial conditions as the actual structure. Once the DSP has the initial conditions the controller is started. The reference model is integrated according to the algorithm described in section 2.3 and control commands are calculated and sent via channel A, through the appropriate circuits (figure 3-19), to the force actuator. The control forces are applied to the structure, causing it to have the desired characteristics of the reference model. Except where noted otherwise, this process is repeated at a rate of $500 \mathrm{~Hz}$. 
Table 3-1

Comparison of Analytical and Experimental

Frequencies for the Slewing Grid Structure

\begin{tabular}{|c||c|c|c||}
\hline \multirow{2}{*}{ Mode \# } & \multicolumn{2}{|c|}{ Frequencies $(\mathrm{Hz})$} & \multirow{2}{*}{ \%Error } \\
\cline { 2 - 3 } & Analytical & Experimental & \\
\hline \hline 1 & 0.42 & 0.42 & 0.00 \\
\hline 2 & 1.37 & 1.45 & 5.52 \\
\hline 3 & 3.00 & 2.88 & 4.17 \\
\hline 4 & 4.47 & 5.39 & 17.07 \\
\hline 5 & 6.02 & 6.41 & 6.08 \\
\hline 6 & 6.69 & 6.88 & 2.76 \\
\hline 7 & 9.79 & 9.05 & 8.18 \\
\hline 8 & 11.52 & 10.18 & 13.16 \\
\hline 9 & 13.11 & 13.56 & 3.32 \\
\hline 10 & 15.35 & 14.90 & 3.02 \\
\hline 11 & 21.16 & 15.37 & 37.67 \\
\hline
\end{tabular}


Table 3-2

Measured and Desired Damping Ratios for the Slewing Grid Structure

\begin{tabular}{|c||c|c|c|}
\hline Mode \# & $\begin{array}{c}\text { Experimental } \\
\text { Frequency } \\
(\mathrm{Hz})\end{array}$ & $\begin{array}{c}\text { Experimental } \\
\text { Damping } \\
\text { Ratio }\end{array}$ & $\begin{array}{c}\text { Desired } \\
\text { Damping } \\
\text { Ratio }\end{array}$ \\
\hline 1 & 0.42 & 0.110 & 0.15 \\
\hline 2 & 1.45 & 0.015 & 0.05 \\
\hline 3 & 2.88 & 0.011 & 0.05 \\
\hline 4 & 5.39 & 0.008 & 0.05 \\
\hline 5 & 6.41 & 0.003 & 0.05 \\
\hline 6 & 6.88 & 0.011 & 0.05 \\
\hline 7 & 9.05 & 0.003 & 0.05 \\
\hline 8 & 10.18 & 0.003 & 0.05 \\
\hline 9 & 13.56 & 0.002 & 0.01 \\
\hline 10 & 14.90 & 0.002 & 0.01 \\
\hline 11 & 15.37 & 0.002 & 0.01 \\
\hline
\end{tabular}




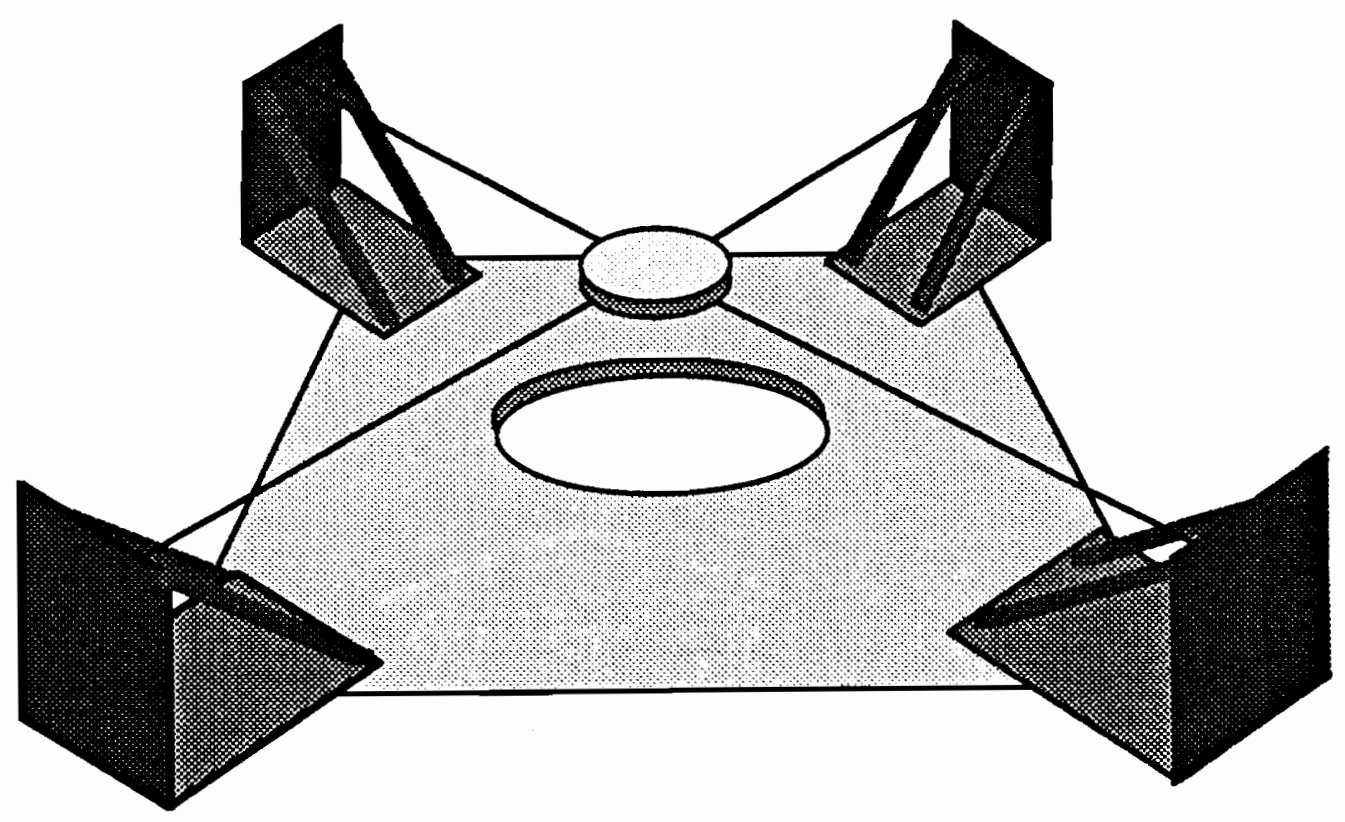

Figure 3-1

Drawing of Suspended Mass System 


$$
\begin{aligned}
& \omega_{n}=5.95 \mathrm{~Hz} \\
& \zeta^{=}=0.0006
\end{aligned}
$$

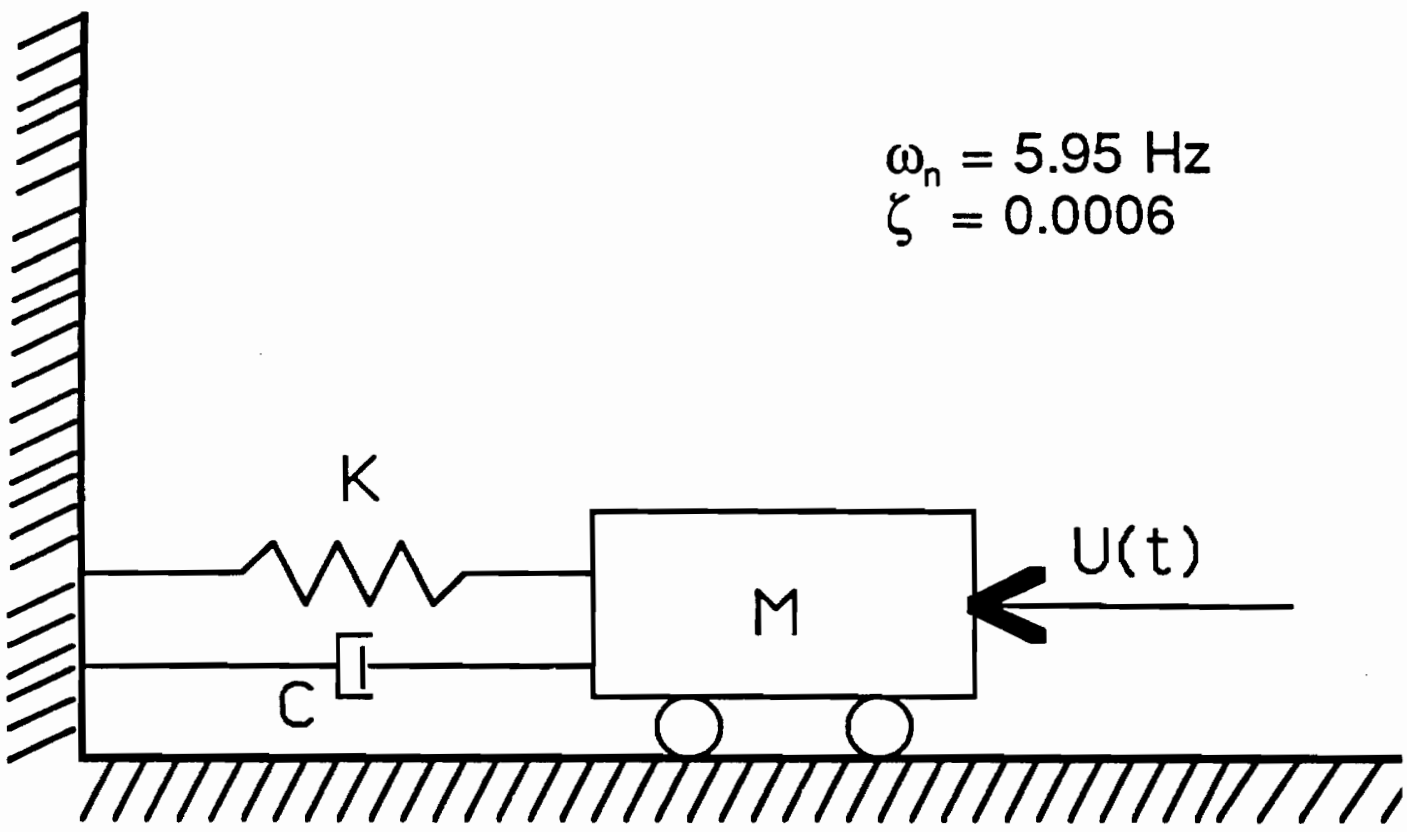

Figure 3-2

Analytical Model of the Suspended Mass System 

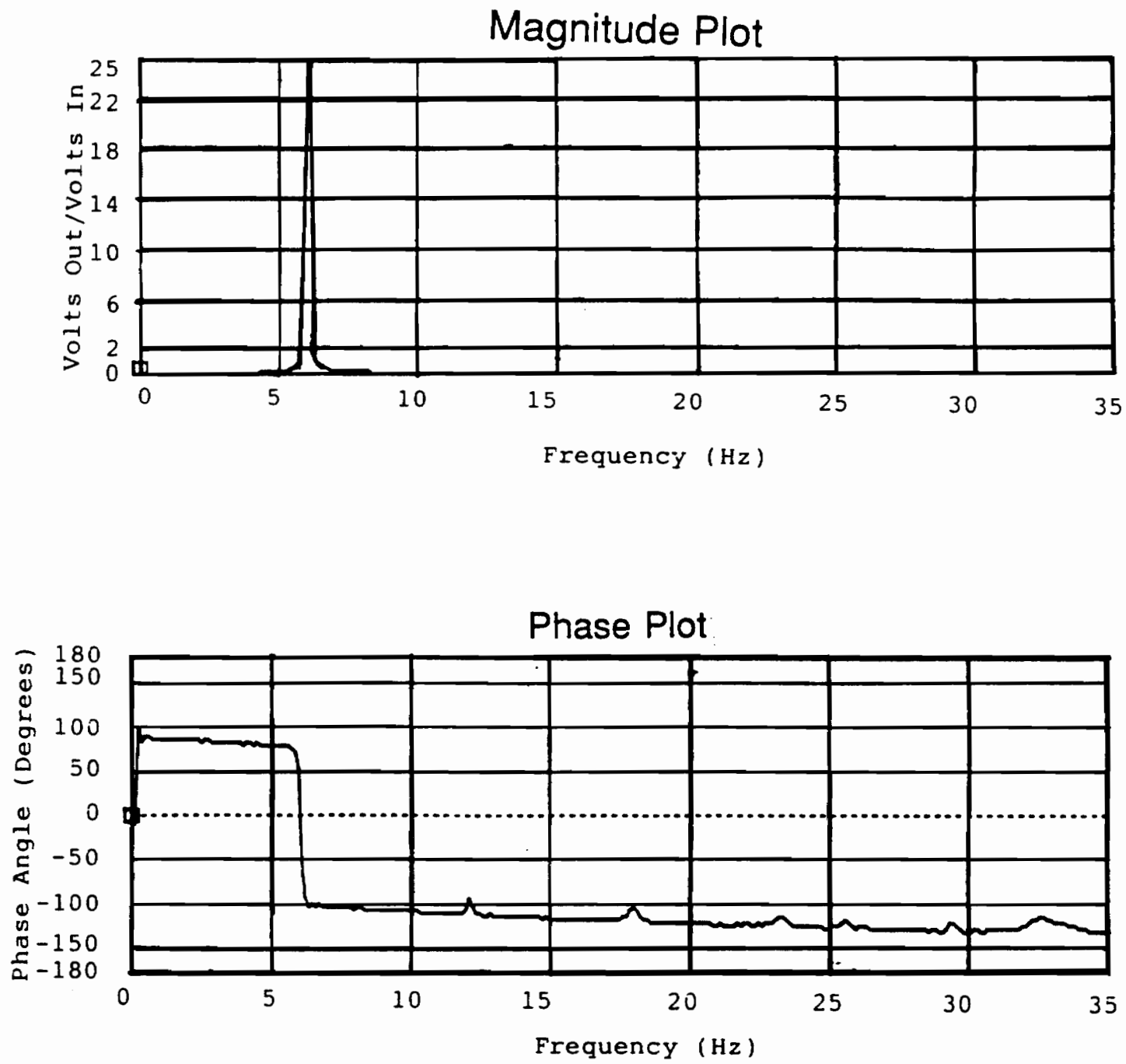

Figure 3-3

Magnitude and Phase Plot of a Velocity Frequency Response

Function for the Suspended Mass System

Force $=[0.07460(\mathrm{lb} / \text { volt })]^{*}[$ Volts In $]$

Velocity $=[34.48(\mathrm{in} / \mathrm{sec}-\text { volt })]^{*}[$ Volts Out $]$ 

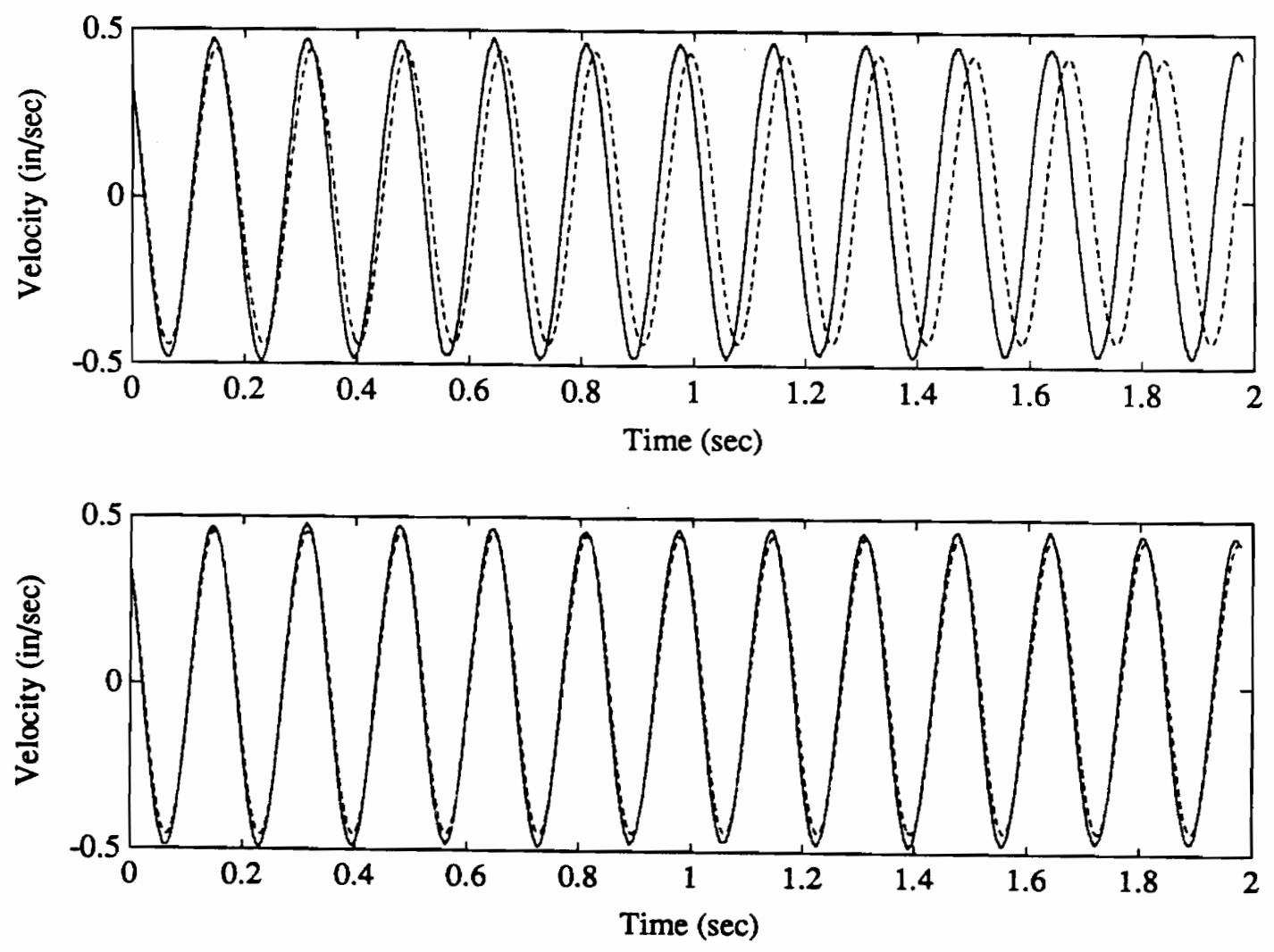

Figure 3-4

Comparison of Analytical and Experimental Free Velocity

Response for the Suspended Mass System

Top: $0.1 \mathrm{~Hz}$ Difference between Experimental and Analytical frequency

Bottom: Experimental $=$ Analytical Frequency 


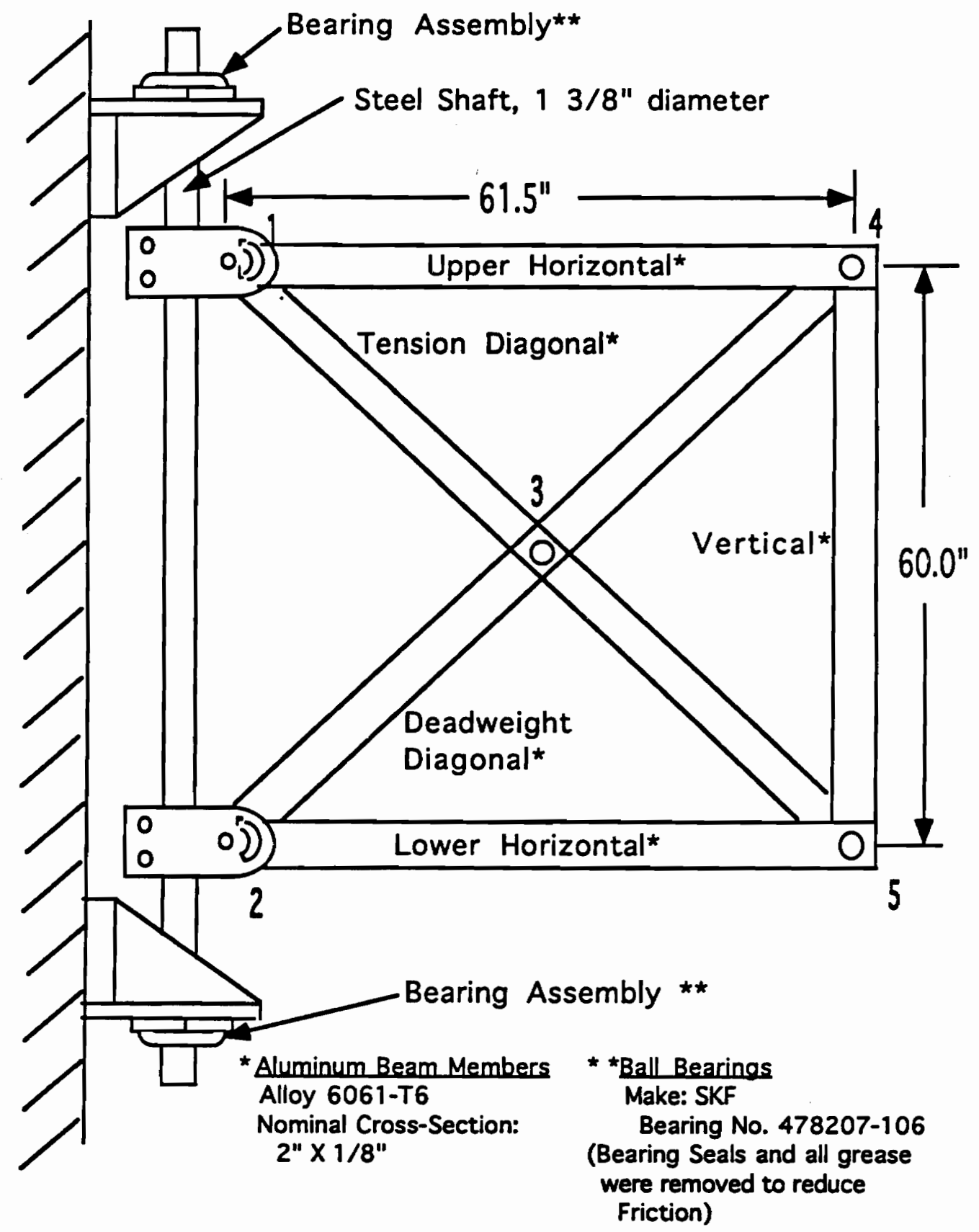

Figure 3-5

Drawing of the Slewing Grid 

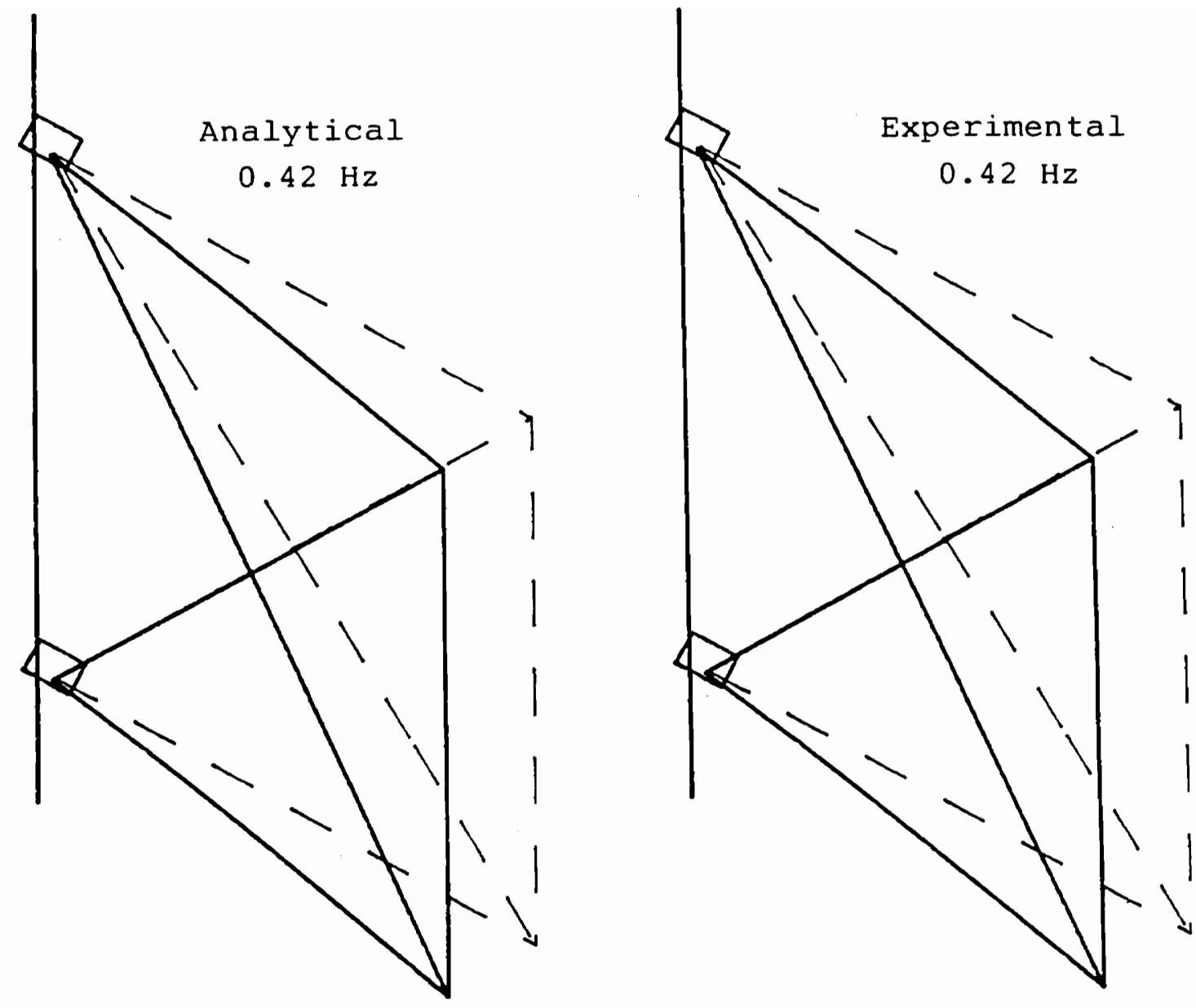

Figure 3-6

Comparison of the Analytical and Experimental First Mode Shape for the Slewing Grid 


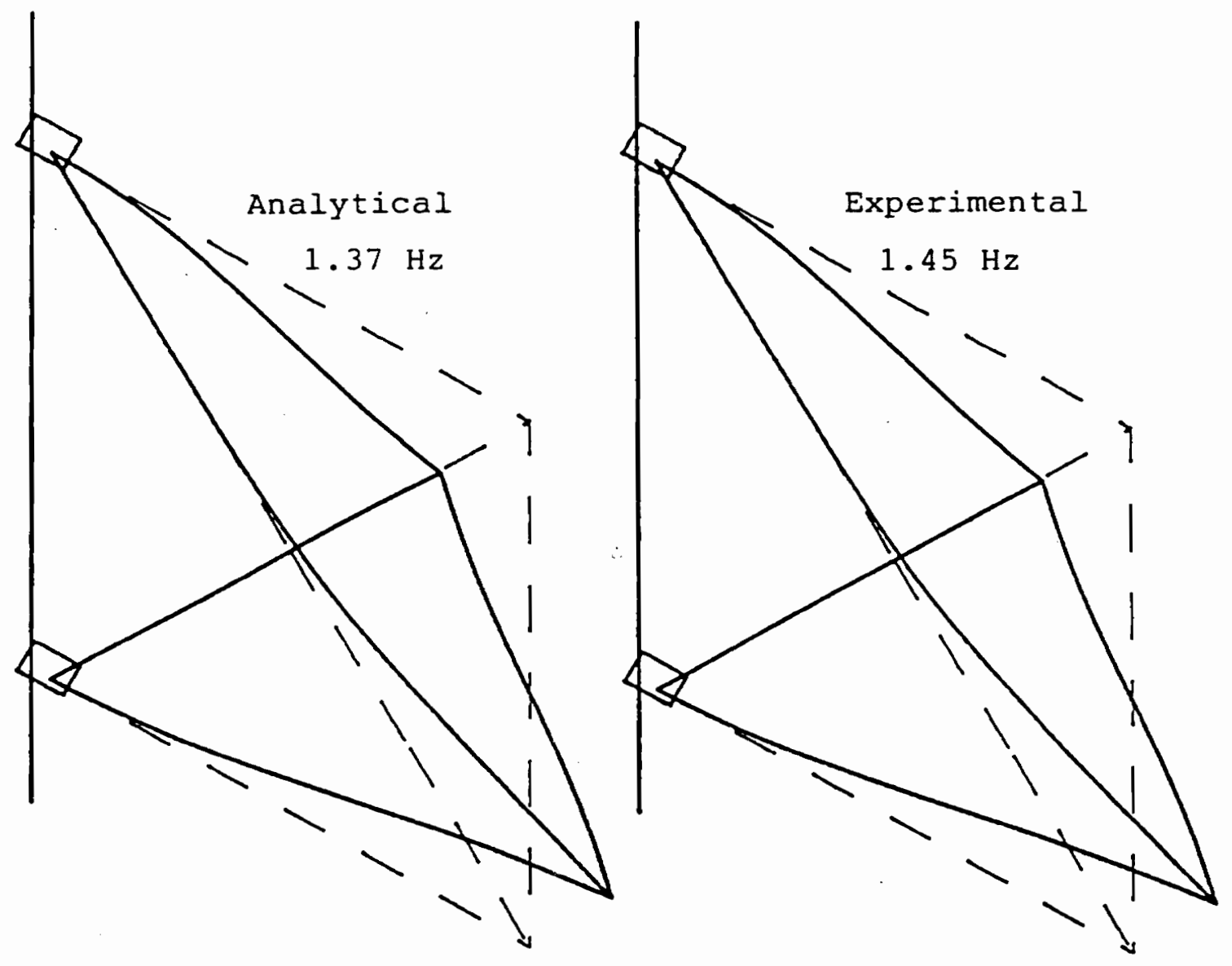

Figure 3-7

Comparison of the Analytical and Experimental

Second Mode Shape for the Slewing Grid 

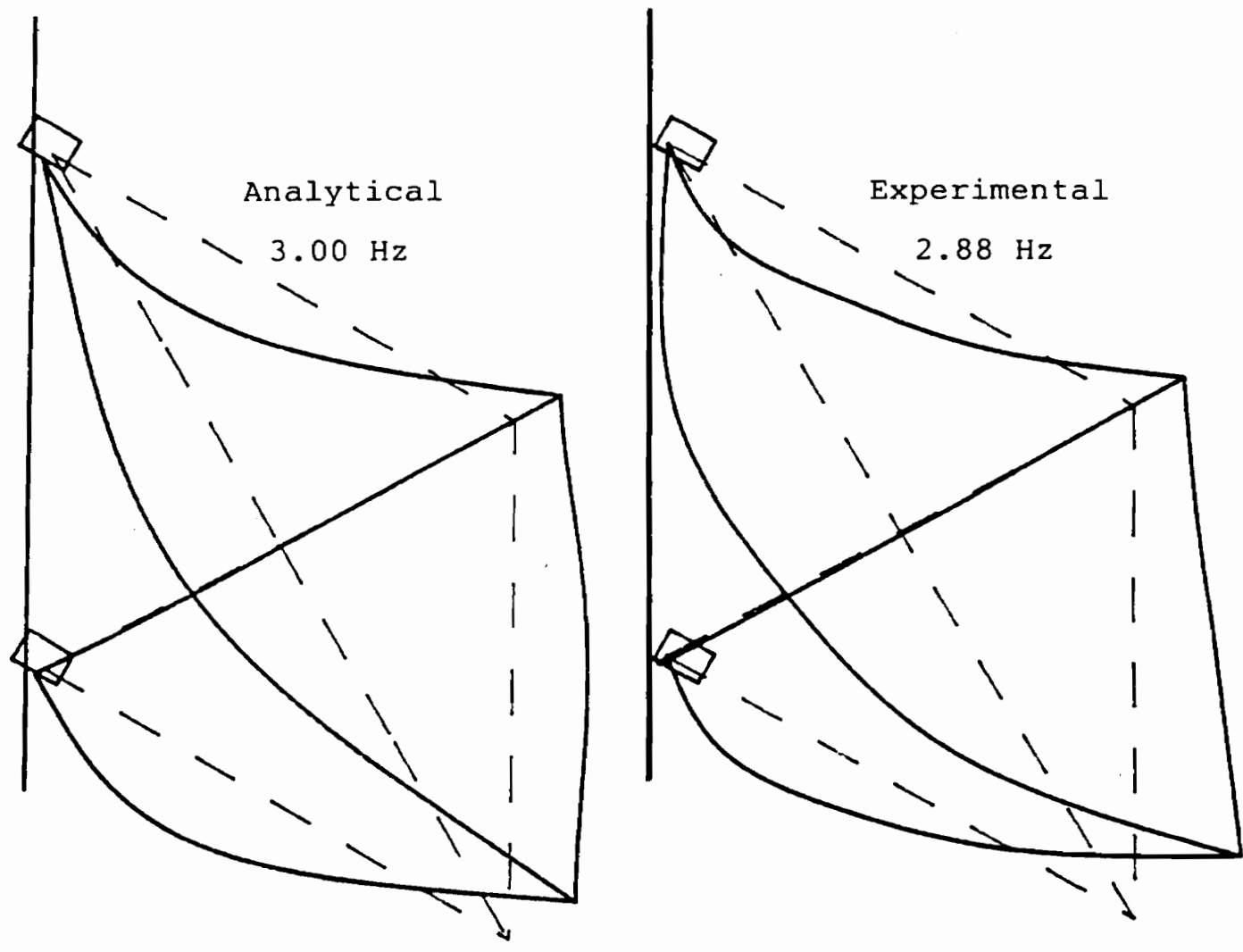

Figure 3-8

Comparison of the Analytical and Experimental

Third Mode Shape for the Slewing Grid 

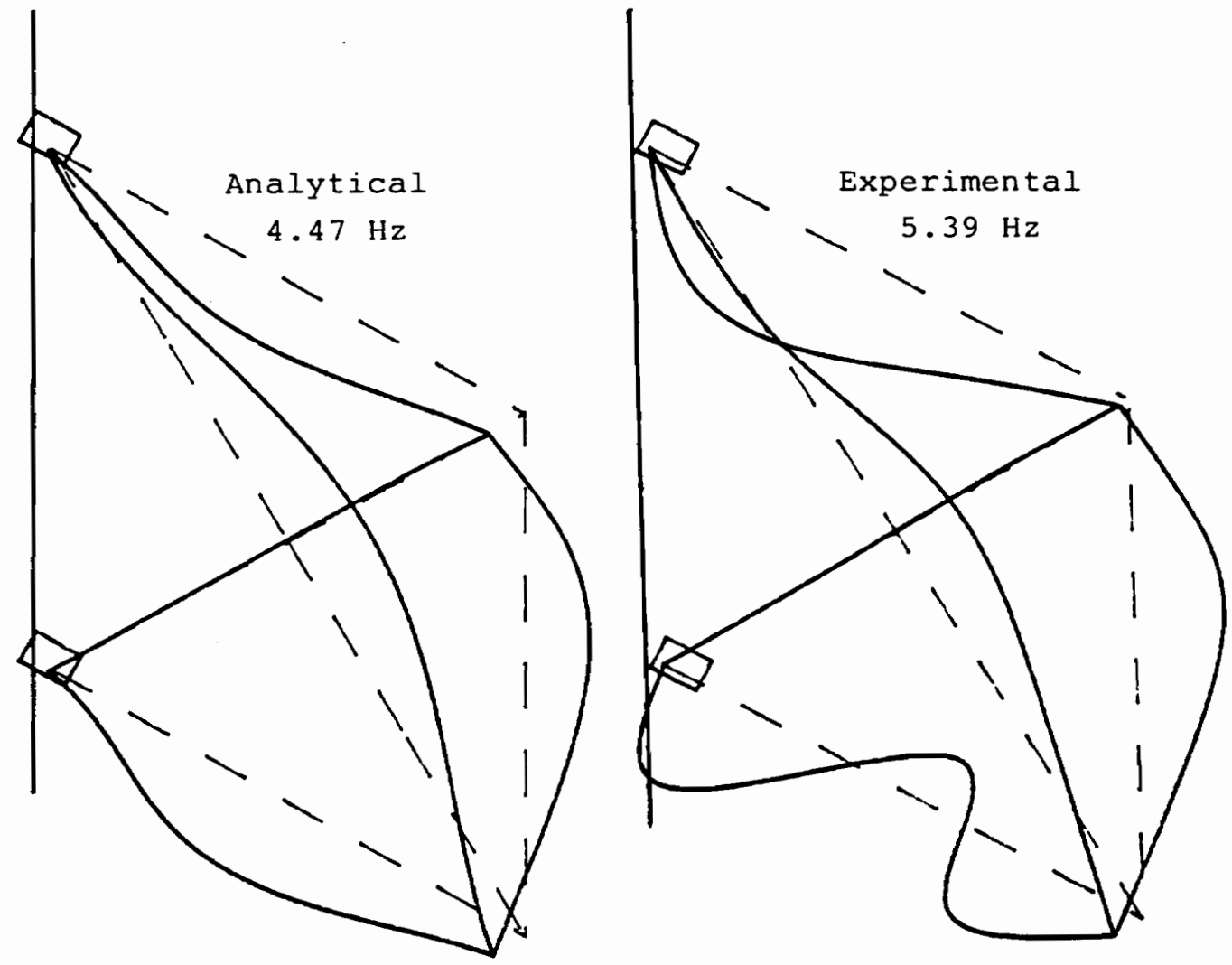

Figure 3-9

Comparison of the Analytical and Experimental

Fourth Mode Shape for the Slewing Grid 

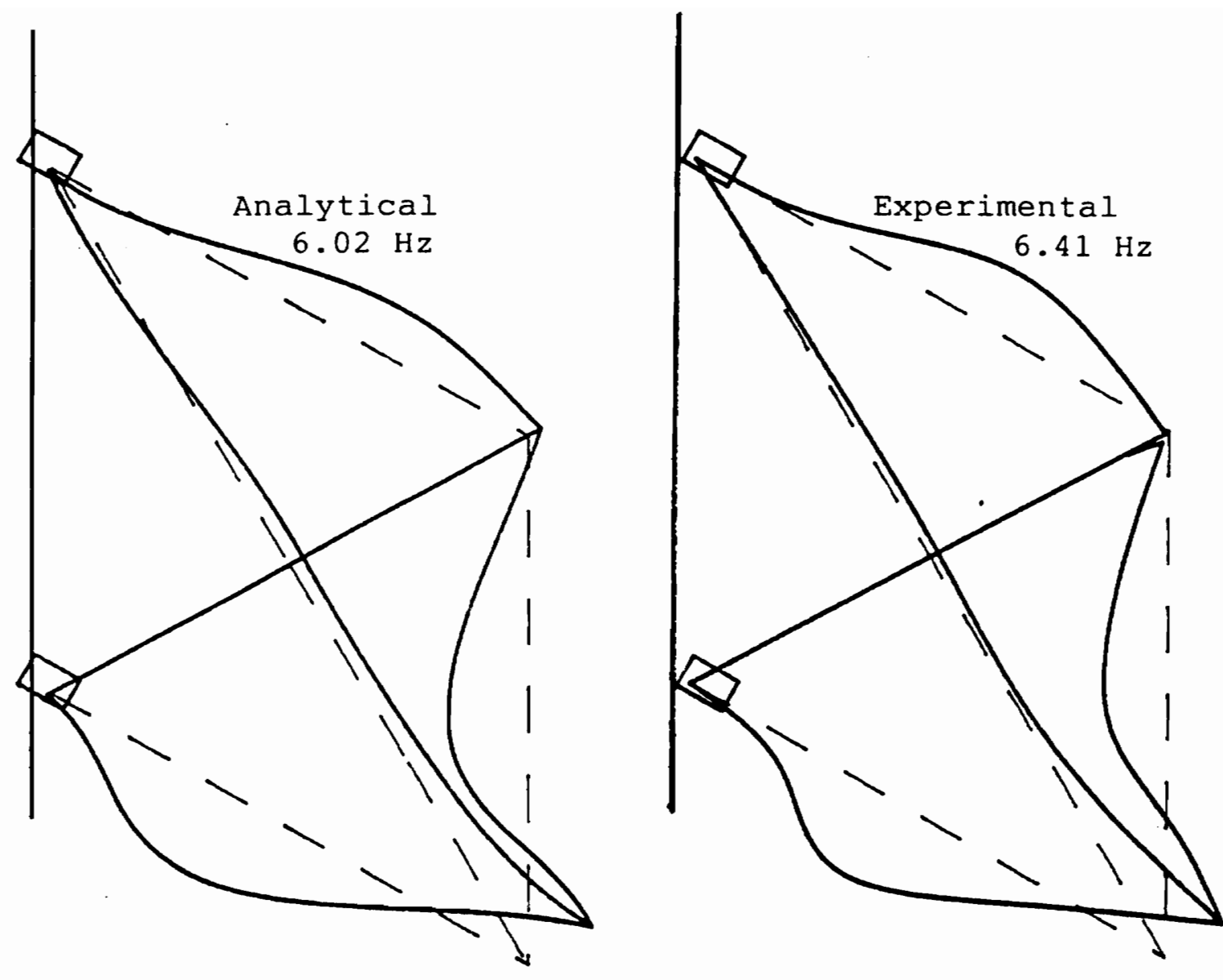

Figure 3-10

Comparison of the Analytical and Experimental Fifth Mode Shape for the Slewing Grid 

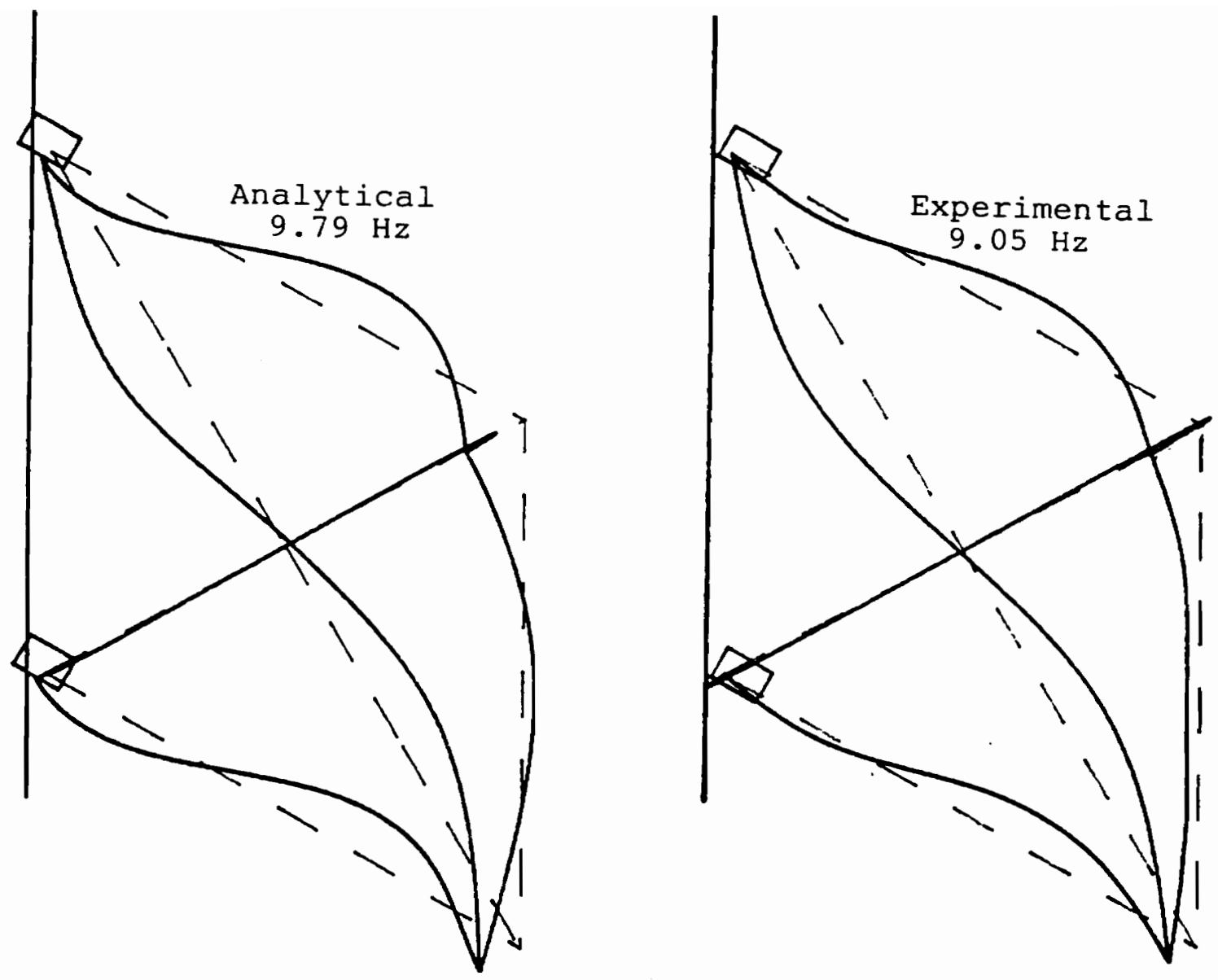

Figure 3-11

Comparison of the Analytical and Experimental

Seventh Mode Shape for the Slewing Grid 

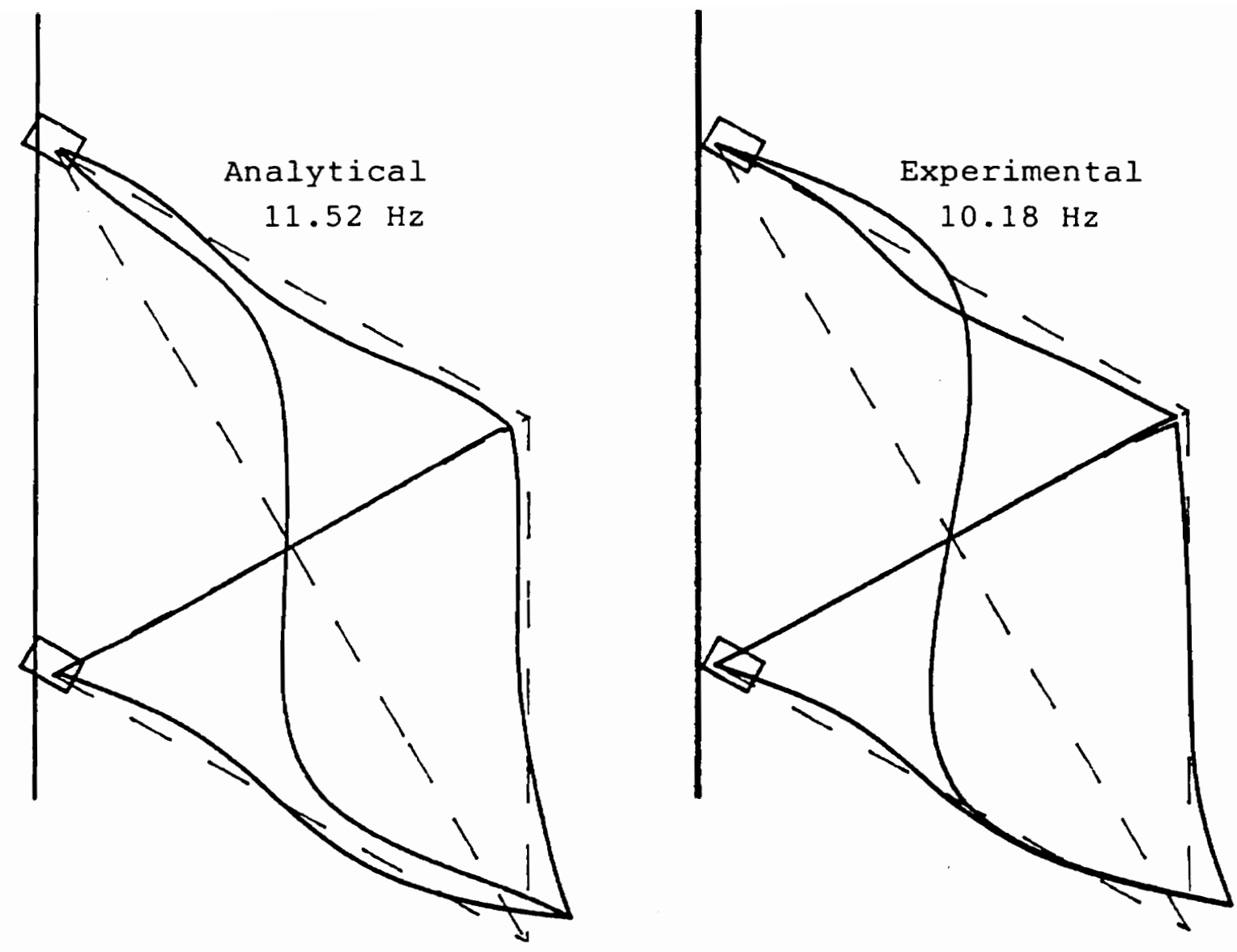

Figure 3-12

Comparison of the Analytical and Experimental

Eighth Mode Shape for the Slewing Grid 


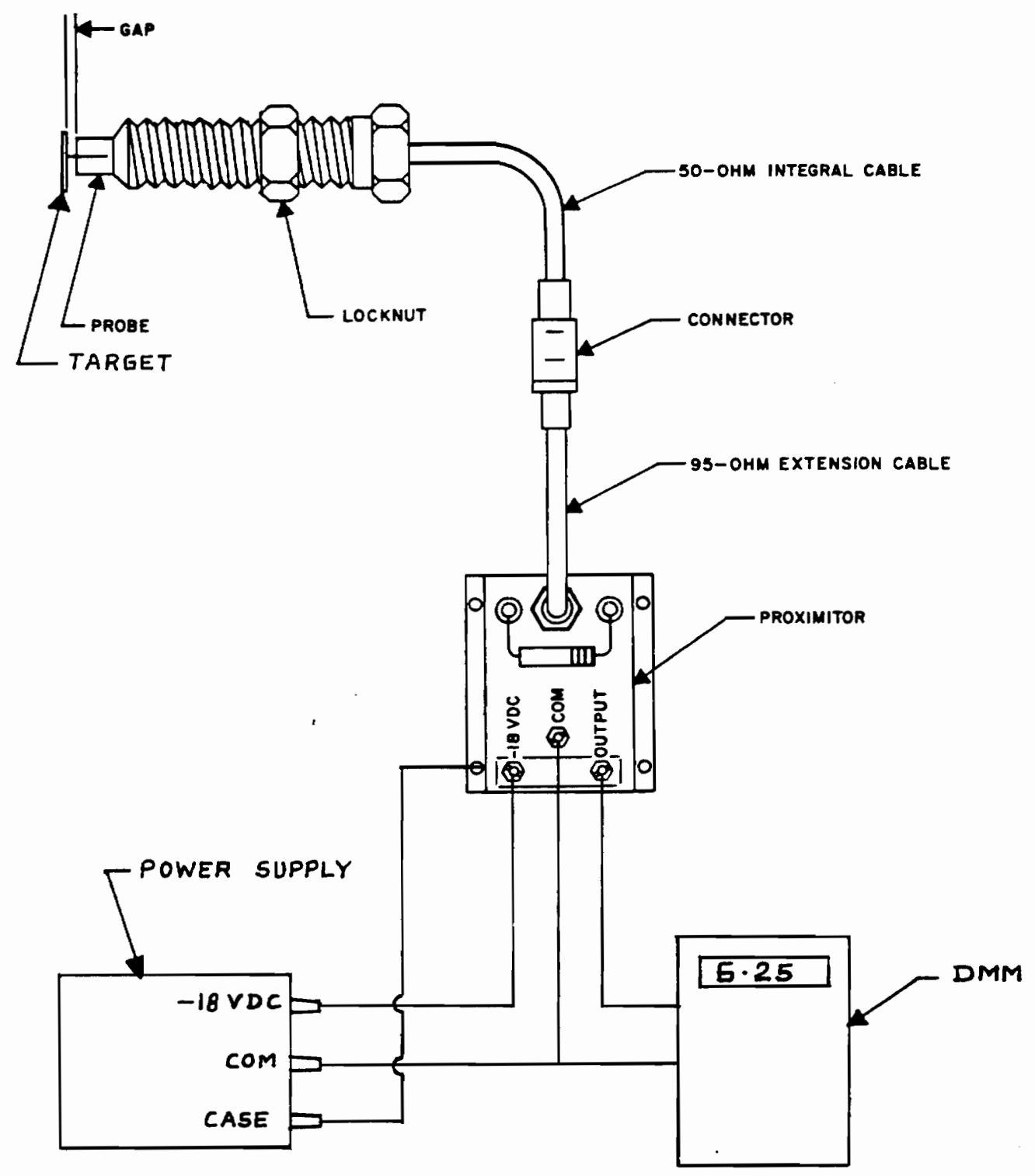

Figure 3-13

Sketch of Displacement Probe by Bently Nevada 


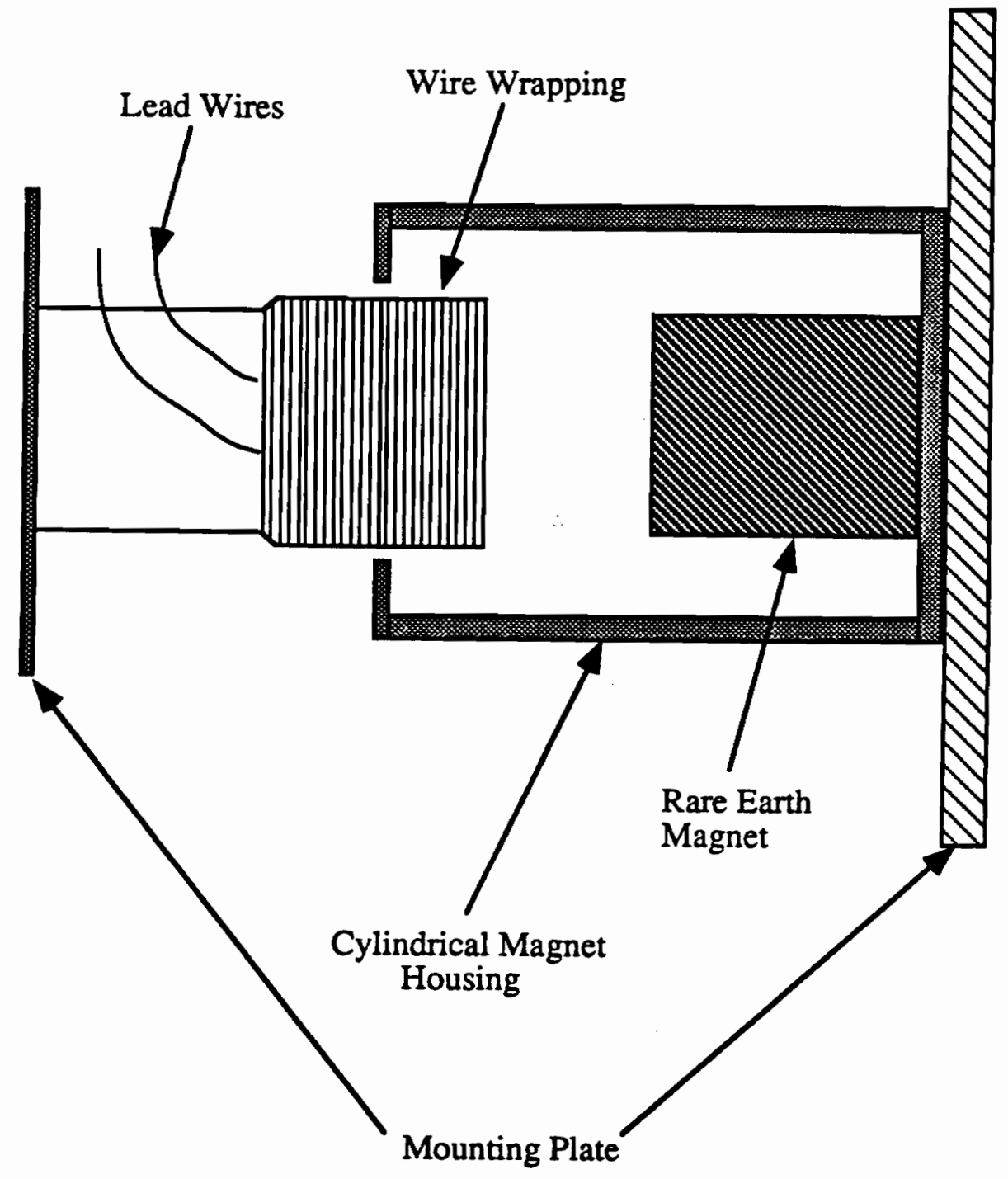

Figure 3-14

Sketch of the Coil and Magnetic Field Assembly Used by Velocity Sensor and Force Actuators 


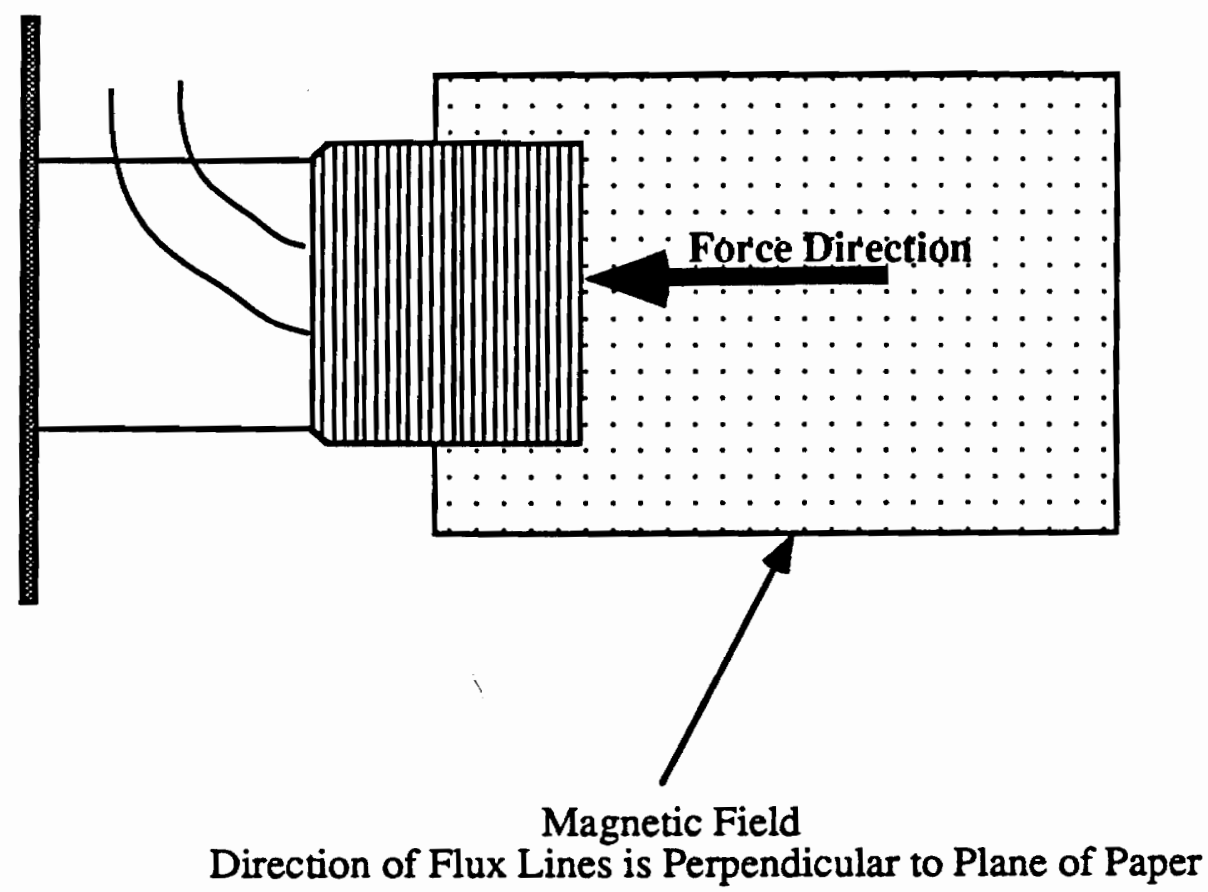

Figure 3-15

Diagram of Force Direction with Respect to the Coil and Magnetic Field 


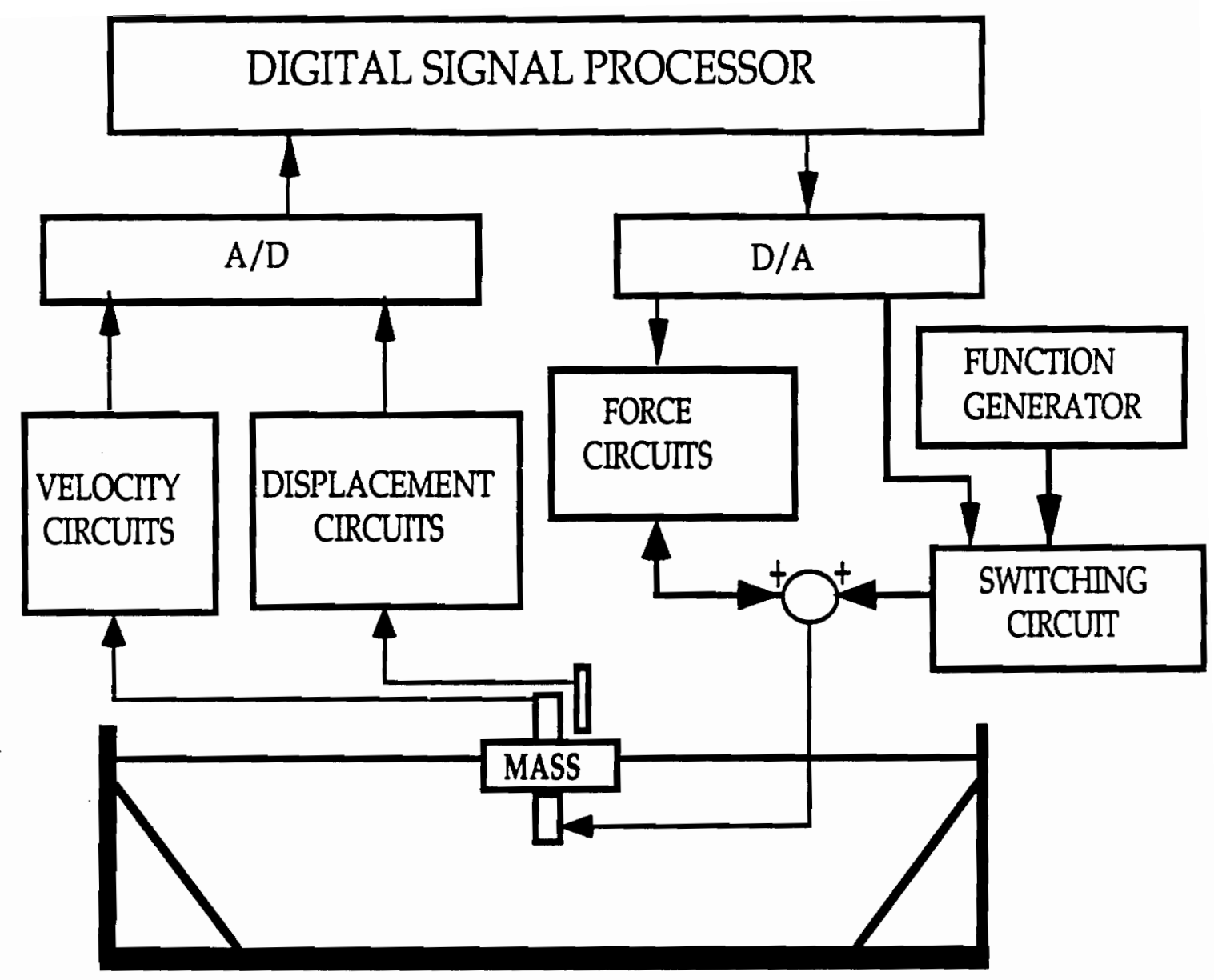

Figure 3-16

Block Diagram of the Experimental Set up for the Suspended Mass 


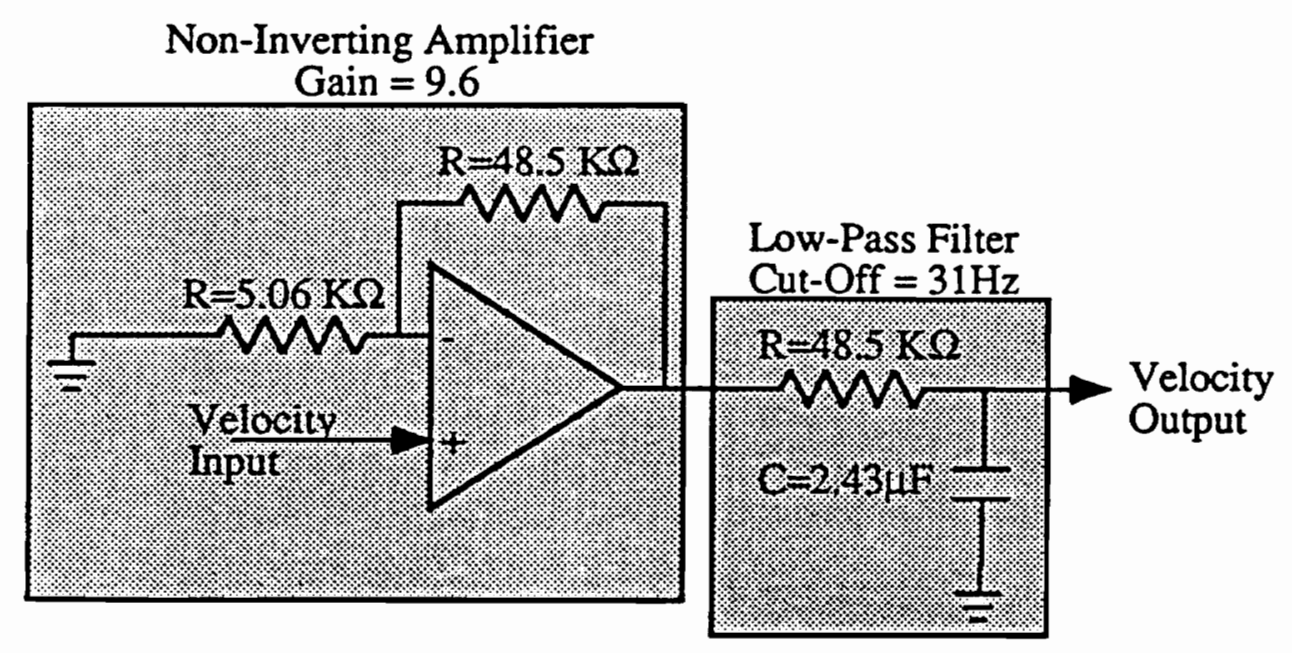

Figure 3-17

Schematic of Electrical Circuits Used for Velocity Signal 


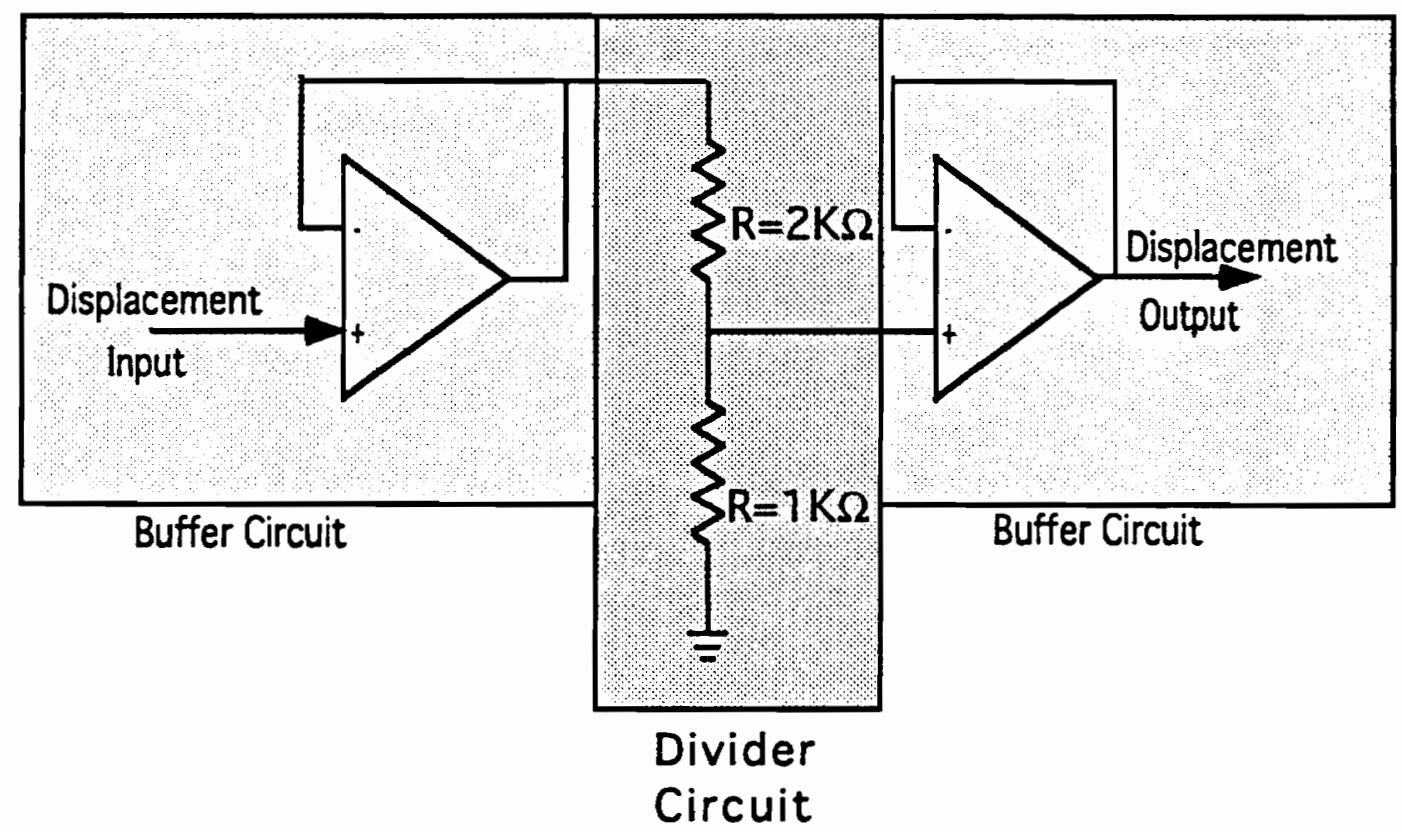

Figure 3-18

Schematic of Electrical Circuits Used for Displacement Signal 


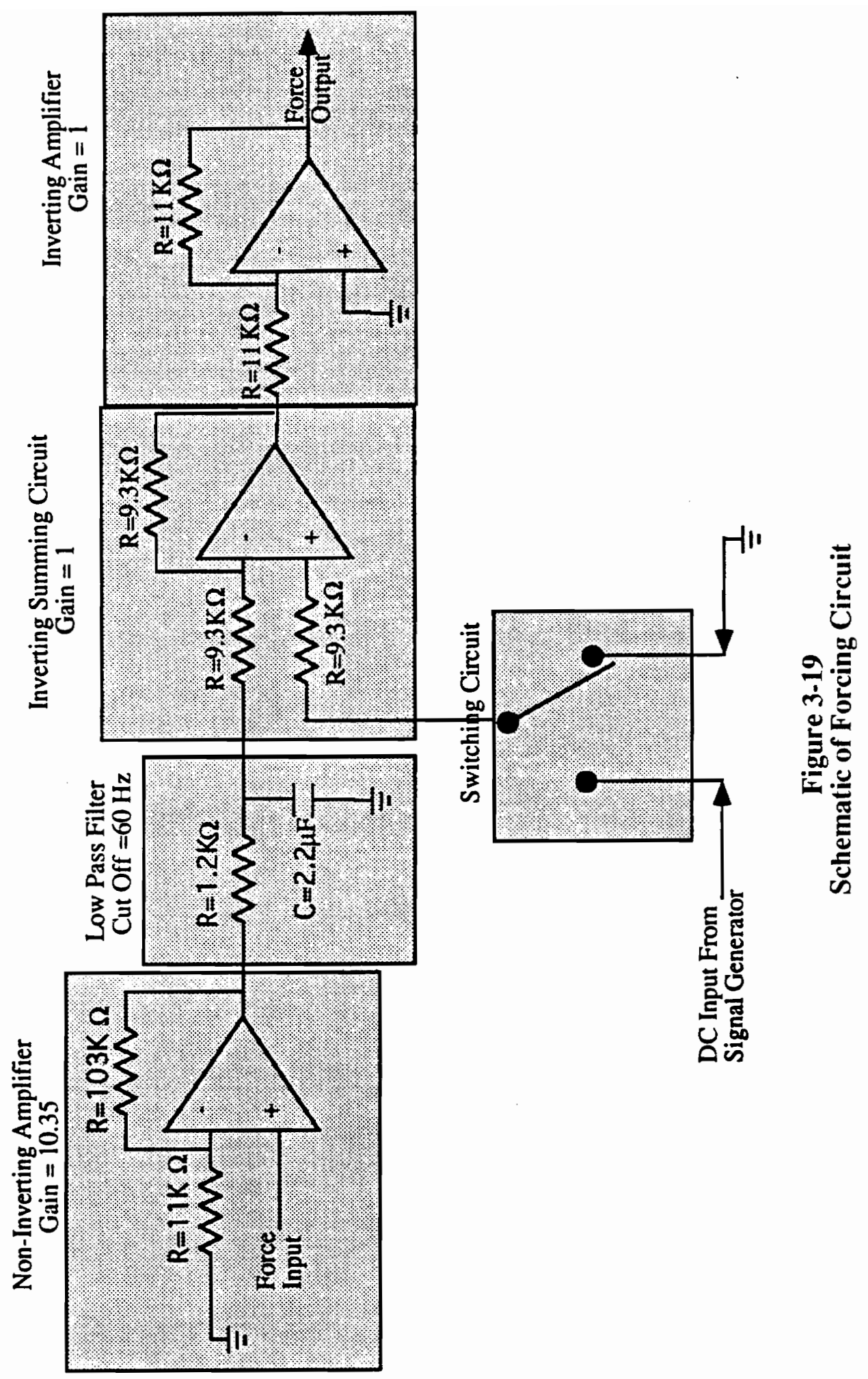




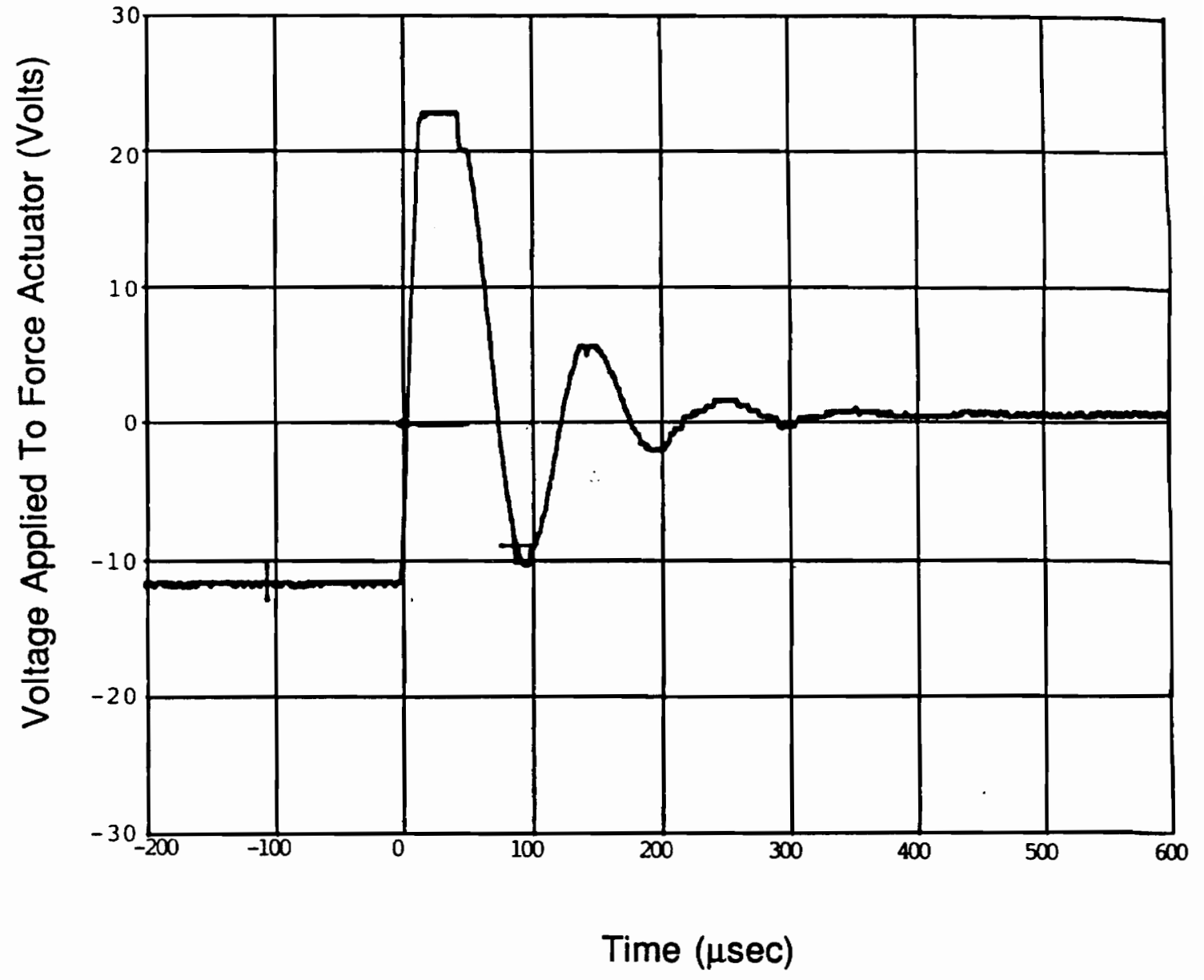

Figure 3-20

Actuator Response when DC Voltage is Set to Zero 


\section{Chapter 4 \\ Effects of Error on MRAC Performance and Control Effort}

As stated in chapter 2, we quantify the difference between the reference model and the actual system in terms of differences in damping, frequencies, and model order. Only the first two aspects are considered in this work. In this chapter it will be shown that the maximum force and control effort both decrease as the reference model damping is increased if the frequency difference between the reference model and the plant is large. It will also be shown that for a constant damping, increasing the frequency difference between the reference model and the plant can dramatically increase the required control forces.

\subsection{Suspended Mass System}

As stated earlier, in order to avoid the effects of the initial transients in the experimental set up the mass was allowed to vibrate freely for about two seconds . It was also discovered experimentally that the amount of delay in starting the control system affected the magnitude of the control effort indices. Several tests were run for different start times and it was found that the maximum force could change by as much as $100 \%$ for a small increase or decrease in start time. Since running hundreds of tests to see how the start time would affect the control effort indices would be too large a task, a brief analytical calculation was made in hopes that the same trends would be found. Figures 4-1 through 4-3 show these analytical calculations for the maximum control force and initial velocity as a function of the controller start time. Figure 4-1 shows that for $\alpha=1.0$ the start time is not significant, because the absolute value of the maximum force is 
constant at $0.025 \mathrm{lbs}$ for all values of start time. However at $\alpha=0.5$ it can be seen, from fig. 4-2, that a \pm 0.5 second change in the start time can cause a dramatic change in the maximum force. For example, a +0.5 second change in the start time decreases the maximum force by more than a factor of 2 . It can also be seen from fig. 4-2 that the greatest value of the maximum force occurs at the peak velocity. However, for $\alpha=1.5$ (fig. 4-3) the minimum force is found when at the peak velocity, but the difference between the maximum and minimum force is only 0.15 to $0.115 \mathrm{lbs}$. The need to use different start times is likely related to differences in the acceleration of the plant and reference model. To eliminate this variation from the results, each test was run at the initial conditions causing the largest maximum force.

To find the start time required to create the appropriate initial conditions and thus produce the maximum force, a series of experimental trial runs were made in which the start time was incremented by 0.005 seconds each run until the maximum force was found. Since the natural frequency of the mass changed over time, a new set of start times was required each time a noticeable change in frequency occurred. Table 4-1 shows the two sets of start times at which most of the tests were run.

Most of the test results were obtained at a required damping ratio of $10 \%$, but it was also of interest to see what effect increasing or decreasing the damping in the reference model would have on the control costs. The effect of increasing or decreasing reference model damping, on maximum force and control effort when using MRAC are shown in figs. 4-4 and 4-5. From these figures it can be seen that, with no frequency difference between the plant and the reference model $(\alpha=1)$, requiring more damping in the reference model has the expected effect of increasing the maximum force and control effort. However, when there are substantial differences in frequency between the reference model and the plant ( $\alpha=0.5$ and $\alpha=1.5$ ), increased damping has the opposite effect. For example, when $\alpha=1.5$ the maximum control force required to achieve $2.5 \%$ damping is 1.5 times larger than required to achieve $20 \%$ damping. The increase in control effort is even more dramatic, with the control effort required for $2.5 \%$ damping 
being 3.7 times larger than for 20\% damping. Similar trends can be seen for $\alpha=0.5$. Although this result is somewhat counter intuitive, one possible explanation follows. When the required damping is very low, the controller adds energy to force the frequency of the plant to follow the frequency of the reference model, and at the same time, removes energy to provide the required damping. As required damping increases, the energy required to force the frequencies to match is reduced, while the energy which must be removed to damp the structure, stays the same or increases only slightly. This is a useful result in that for a given design if the objective was to reduce the control forces, one approach likely to be considered would be to reduce the damping requirements. However, from figs. 4-4 and 4-5 it is seen that when substantial frequency differences exist, this may have just the opposite effect.

The effects of frequency differences for constant required damping are somewhat more predictable, and are shown to always increase as the error between the two model increases. Figures 4-6 through 4-8 show typical velocity responses of the actual system and the reference model for three values of the reference model frequency. As expected, MRAC does an excellent job at forcing the actual structure to follow the reference model. For $\alpha=1.0$, (fig. 4-6) the frequency of the reference model matches the frequency of the actual structure. Therefore, all the controller is required to do is provide $10 \%$ damping to a system where only $0.067 \%$ inherent damping exists. It can also be seen that the analytical velocity response matches the experimental results closely.

Figure 4-7 shows the controller forcing the $6 \mathrm{~Hz}$ structure to vibrate as if it had a 7.8 $\mathrm{Hz}$ fundamental frequency with $10 \%$ damping. While the initial overshoot of the actual structure on the first cycle is almost $30 \%$ larger than the reference model, the overall performance of the controller is good. Once the controller locks onto the reference model, it does a good job at driving the error (equation 2-5) between the two systems to zero. As with the $\alpha=1.0$ case the analytical velocity response is essentially the same as the experimental velocity after 1 cycle. However, it is interesting to notice that at 1 cycle the experimental velocity response is closer to the reference model than the analytical 
response, while just the opposite was true at $1 / 2$ of a cycle.

Figure 4-8 shows the same experimental and analytical trends for the $\alpha=0.7$ case. When the controller is first turned on, it takes a short time to lock onto the reference model. However it eventually does lock on and does an excellent job at providing $10 \%$ damping at a frequency different from the natural frequency of the actual structure. A comparison of figs. 4-7 and 4-8 reveals the actual structure leads the reference model for $\alpha=1.3$ while it lags for $\alpha=0.7$. This difference may be related to the time lag inherent in the digital implementation using the DSP board rather than the controller itself.

Even though the controller does a good job at locking onto the reference model when differences between the two are introduced, table 4-2 shows that increasing the frequency difference between the reference model and the actual system increases both the maximum force $\left(U \mathrm{p}_{\max }\right)$ and the control effort $(\mathrm{CE})$. Although the correlation between experiments and simulation is not as good as the velocity response, both sets of maximum forces and control efforts show the same trends. Though this increase in maximum force and control effort might be expected, the real issue is its magnitude.

We define the force and control effort magnification factors as $U p_{\max } /\left(U p_{\max }\right)_{\alpha=1}$ and $\mathrm{CE} /(\mathrm{CE})_{\alpha=1}$. Figures 4-9 and 4-10 show the magnification factors as a function of the frequency difference between the reference model and the plant. Figure 4-9 shows that for $\alpha=0.5$, the experimental $U p_{\max }$ increases by a factor of 4.1 , while for $\alpha=1.5, U \mathrm{p}_{\max }$ increases by a factor of 6 . The same trends occur for the analytical curve except the magnifications are more symmetric. One final observation from fig. 4-9 is that the magnification factors predicted by the analysis are generally very close to or conservative when compared to the experimental results. This is a desirable result for designing a controller using analytical simulations.

Magnification of the control effort (fig. 4-10) follows the same general trends except the maximum magnification occurs for both the analytical and experimental case $\alpha=0.5$. For $\alpha=0.5$, the analytical CE is 9.5 times larger than the perfect- knowledge case ( $\alpha=$ 1.0), and the experimental $\mathrm{CE}$ is 8.1 times larger. As is the maximum force case, the 
analytical calculations are very close or conservative when compared to the experimental results.

It is noted, that although the trends are the same, the magnitude of the results of table 4-2 and figs. 4-9 and 4-10 are slightly different than those presented in ref. 49. This is because the experimental set-up was dismantled and put back together. Although the experimental and analytical correlations are better for the results presented here, this may not always be the case, due to random in putting the experiment together and re-building all the signal conditioning circuits.

\subsection{Slewing Grid Structure}

As stated earlier, the control results presented for the slewing grid are strictly analytical in nature. However, experimental measurements were used to develop the corrected model of the structure, and the reader is reminded that the reference model chosen for the slewing grid is a linear combination of the corrected model and the standard FEM (see eq. 3-11). From eq. 3-11 it is seen that $\alpha=1$ is the perfect modeling case with added damping in the reference model (see table 3-2 for reference model damping ratios), and $\alpha=0$ is the case of maximum difference between the reference model and the plant. As in the Suspended Mass example, the initial conditions for the Slewing Grid simulation consisted of a specified displacement with zero initial velocity at all DOF. Both the reference model and the actual model were given the same initial conditions. In addition to varying the parameter $\alpha$, two different initial conditions were tried. In the first simulation, the initial displacement condition matched the second mode shape (fig. 3-7), and in the second simulation, the structure was deformed into the theoretical fourth mode shape and released (see fig. 3-9).

Table 4-3 shows the value of the maximum force at each joint and the control effort for the entire structure for second mode initial conditions, and fig. 4-11 shows the magnification of $\mathrm{Up}_{\max }$ at each joint as the difference between the two models gets larger. For the maximum difference case, $\alpha=0.0$ (see equation 3-11), the maximum force at 
joint 3 (see fig. 3-5) is increased by a factor of 10 . While not as large an increase occurred in joints $4 \& 5$, a 3 fold increase over the perfect knowledge case is still significant considering the differences that were introduced.

Table 4-4 and fig. 4-12 show trends similar to the first case. Figure 4-12 shows that for the $\alpha=0$ case, the maximum force at joint 3 increased by a factor of 7 , and the maximum force at joints 4 and 5 increased by a factor of 1.5. It is interesting to note that even though the fourth mode was difficult to model in the FEM and had a $25 \%$ error in natural frequency, the magnifications for this case are not as large as the case where the second mode was used as an initial condition. For example, at $\alpha=0.0$ the maximum force at joint 3 only increased by a factor of 7 and the magnifications at joints 4 and 5 were only 1.5. This is a perplexing result because the second mode shape (used in the first case) is almost perfectly modeled by the FEM even before correction, whereas, as stated earlier, the fourth mode was very poorly modeled.

Finally, fig. 4-13 shows that the magnification of the control effort for both cases is nearly the same and is smaller than for the Suspended Mass. Both curves increase linearly from $\alpha=1.0$ (the perfect knowledge case) where the magnification is by definition 1 , to the maximum error case $(\alpha=0.0)$ where the control effort was increased by a factor of about 5 . It is interesting that for the more complex structure, the magnification of the maximum force was larger than for the simpler Suspended Mass, while just the opposite is true of the magnification of the control effort. The cause of this is not easy to determine since there is no way to accurately compare the amount of difference introduced into the Slewing Grid with the frequency difference introduced into the Suspended Mass.

As in the Suspended Mass example it was desirable to see the effect of increased damping for the case of maximum difference between the structure and reference model. Two additional simulations were run, using the second mode as an initial condition, where the damping values of table 3-2 were doubled and then tripled for the case of maximum difference between the reference model and the plant $(\alpha=0)$ (see table 4-5). Although 
the changes in maximum force and control effort were not as large, the same trends were apparent. For example, table 4-5 shows that when the damping was tripled, the total control effort of the three actuators decreased by a factor of 1.3 , and the maximum force required at joint 3 decreased by $7.5 \%$. However, the maximum forces at joints 4 and 5 increased slightly. These increases are most likely due to re-distribution of control forces. As the peak of the frequency response plot is flattened out, the mode shapes change, and the actuators which have the greatest effect on the damping of this new mode must also change. Since the magnitude of the forces at joints 4 and 5 are $1 / 3$ as large as the forces at joint 3, the conclusion of decreased control forces and control effort with increased damping is also valid for the more complicated Slewing Grid. 
Table 4-1

Start Times (sec) for the Two Natural Frequencies

That Most Tests Were Run at (Suspended Mass)

\begin{tabular}{|c||c|c|}
\hline \multirow{2}{*}{ Alpha } & \multicolumn{2}{c|}{ Natural Frequency of Actual System } \\
\hline 0.5 & $\omega_{\mathrm{n}}=5.953 \mathrm{~Hz}$ & $\omega_{\mathrm{n}}=6.025 \mathrm{~Hz}$ \\
\hline 0.6 & 2.072 & 2.040 \\
\hline 0.7 & 2.070 & 2.042 \\
\hline 0.8 & 2.078 & 2.046 \\
\hline 0.9 & 2.026 & 2.030 \\
\hline 1.0 & 2.010 & 2.025 \\
\hline 1.1 & 2.074 & 2.060 \\
\hline 1.2 & 2.086 & 2.055 \\
\hline 1.3 & 2.092 & 2.065 \\
\hline 1.4 & 2.098 & 2.065 \\
\hline 1.5 & 2.014 & 2.055 \\
\hline
\end{tabular}


Table 4-2

Comparison of Experimental and Analytical Maximum

Force and Total Control Effort (Suspended Mass)

\begin{tabular}{|c||c|c||c|c||}
\hline \multirow{2}{*}{ Alpha } & \multicolumn{2}{|c|}{ Maximum Force (lbs) } & \multicolumn{2}{c|}{ Control Effort $\left(\mathrm{lbs}-\mathrm{s}^{1 / 2}\right)$} \\
\cline { 2 - 5 } & Experiment & Theory & Experiment & Theory \\
\hline 0.5 & 0.127 & 0.168 & 0.0616 & 0.0854 \\
\hline 0.6 & 0.102 & 0.131 & 0.0399 & 0.0550 \\
\hline 0.7 & 0.086 & 0.096 & 0.0255 & 0.0346 \\
\hline 0.8 & 0.050 & 0.038 & 0.0145 & 0.0134 \\
\hline 0.9 & 0.034 & 0.026 & 0.0088 & 0.0099 \\
\hline \hline 1.0 & 0.031 & 0.028 & 0.0076 & 0.0089 \\
\hline \hline 1.1 & 0.031 & 0.037 & 0.0081 & 0.0106 \\
\hline 1.2 & 0.041 & 0.060 & 0.0117 & 0.0177 \\
\hline 1.3 & 0.070 & 0.089 & 0.0192 & 0.0262 \\
\hline 1.4 & 0.135 & 0.122 & 0.0275 & 0.0353 \\
\hline 1.5 & 0.186 & 0.162 & 0.0367 & 0.0449 \\
\hline
\end{tabular}


Table 4-3

Analytical Control Effort and Maximum Force for the Slewing Grid.

Initial Conditions $=$ Second Mode Shape

\begin{tabular}{|c|c|c|c|c||}
\hline \multirow{2}{*}{ ALPHA } & \multicolumn{3}{|c|}{ MAXIMUM FORCE (lbs) } & $\begin{array}{c}\text { CONTROL } \\
\text { EFFORT } \\
\text { lbs-sec }\end{array}$ \\
\cline { 2 - 4 } & JOINT \#3 & JOINT \#4 & JOINT \#5 & 0.021 \\
\hline \hline 1.00 & 0.0182 & 0.022 & 0.021 & 0.033 \\
\hline 0.75 & 0.0596 & 0.028 & 0.025 & 0.055 \\
\hline 0.50 & 0.1090 & 0.038 & 0.035 & 0.086 \\
\hline 0.25 & 0.1520 & 0.048 & 0.045 & 0.112 \\
\hline 0.00 & 0.1825 & 0.057 & 0.058 & \\
\hline
\end{tabular}


Table 4-4

Analytical Control Effort and Maximum Force for the Slewing Grid.

Initial Conditions $=$ Fourth Mode Shape

\begin{tabular}{|c|c|c|c||c|}
\hline \multirow{2}{*}{ ALPHA } & \multicolumn{3}{|c|}{ MAXIMUM FORCE (lbs) } & $\begin{array}{c}\text { CONTROL } \\
\text { EFFORT } \\
\text { lbs-sec }^{1 / 2}\end{array}$ \\
\cline { 2 - 4 } & JOINT \#3 & JOINT \#4 & JOINT \#5 & 0.59 \\
\hline 1.00 & 0.44 & 0.68 & 0.58 & 0.72 \\
\hline 0.75 & 0.58 & 0.78 & 0.64 & 1.21 \\
\hline 0.50 & 1.19 & 0.93 & 0.72 & 2.28 \\
\hline 0.25 & 2.42 & 1.04 & 0.89 & 3.01 \\
\hline 0.00 & 3.12 & 1.04 & 0.90 & \\
\hline
\end{tabular}


Table 4-5

Analytical Control Effort and Maximum Force for the Slewing Grid at Maximum Difference $(\alpha=0.0)$ Between the Plant and Reference Model Initial Conditions $=$ Second Mode Shape

\begin{tabular}{|c|c|c|c|c|}
\hline \multirow{2}{*}{$\begin{array}{l}\text { Required } \\
\text { Damping } \\
\end{array}$} & \multicolumn{3}{|c|}{ Maximum Force (lbs) } & \multirow{2}{*}{$\begin{array}{c}\text { Control } \\
\text { Effort } \\
\text { lbs-sec }^{1 / 2} \\
\end{array}$} \\
\hline & Joint \#3 & Joint \#4 & Joint \#5 & \\
\hline $\begin{array}{c}\text { Baseline } \\
\text { (Table 3-2) }\end{array}$ & 0.181 & 0.057 & 0.057 & 0.0116 \\
\hline $\begin{array}{l}2 \text { Times } \\
\text { Baseline }\end{array}$ & 0.174 & 0.072 & 0.061 & 0.0078 \\
\hline $\begin{array}{l}3 \text { Times } \\
\text { Baseline }\end{array}$ & 0.168 & 0.089 & 0.073 & 0.0067 \\
\hline
\end{tabular}




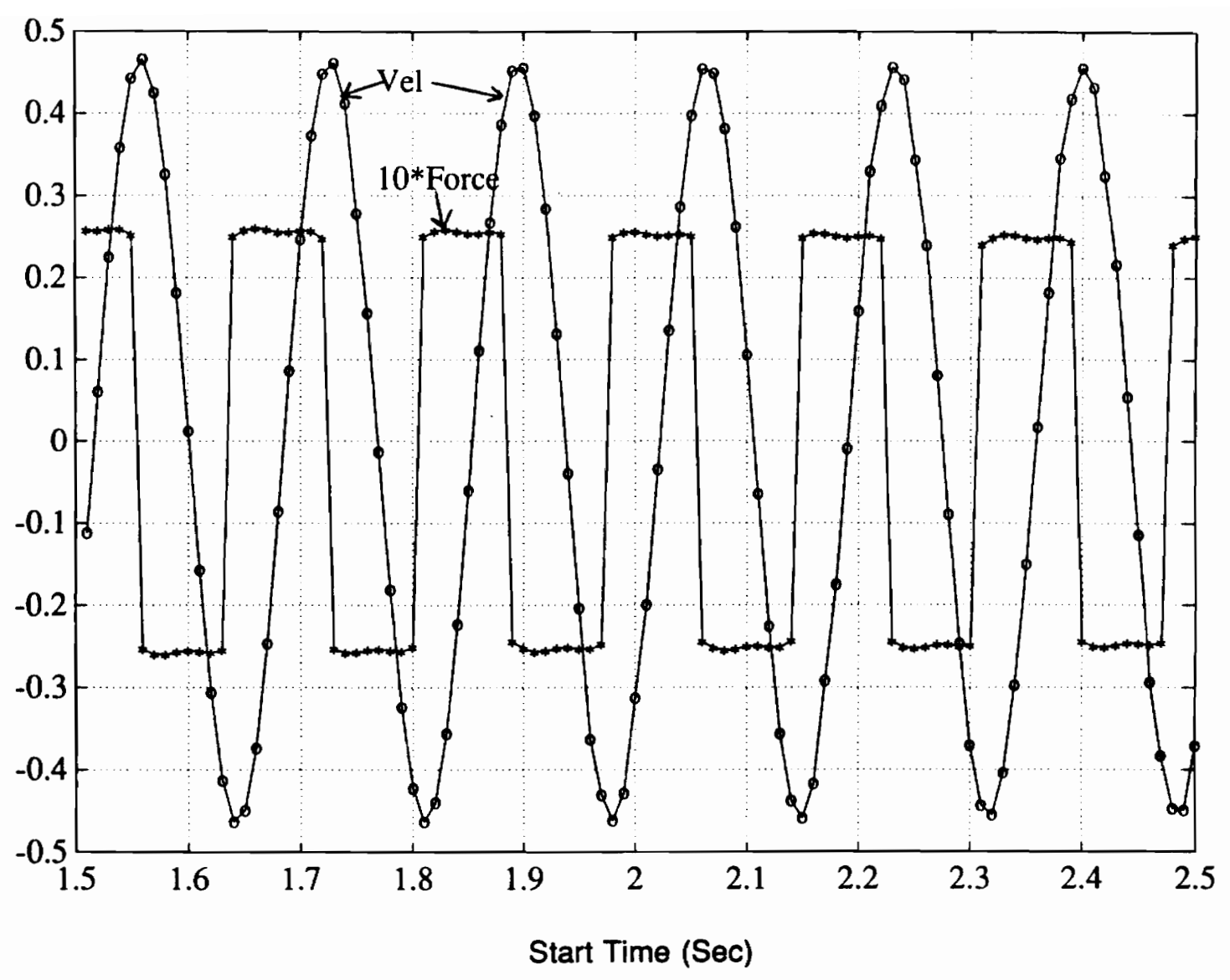

Figure 4-1

Analytical Maximum Force (Lbs) and Initial Velocity (in/sec) for the Suspended Mass Versus Start Time $(\alpha=1.0)$ 


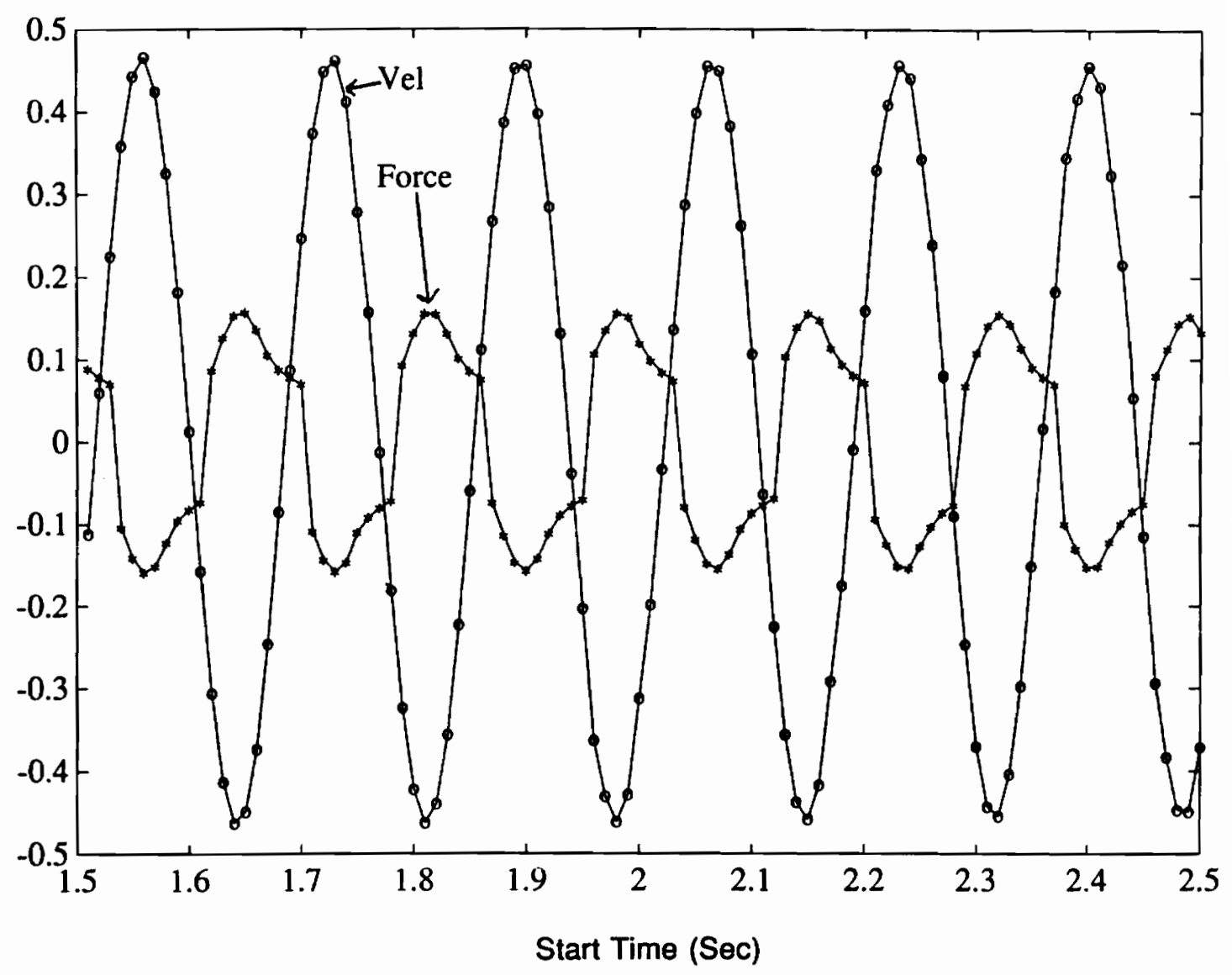

Figure 4-2

Analytical Maximum Force (lbs) and Initial Velocity (in/sec) for Suspended Mass Versus Start Time $(\alpha=0.5)$ 


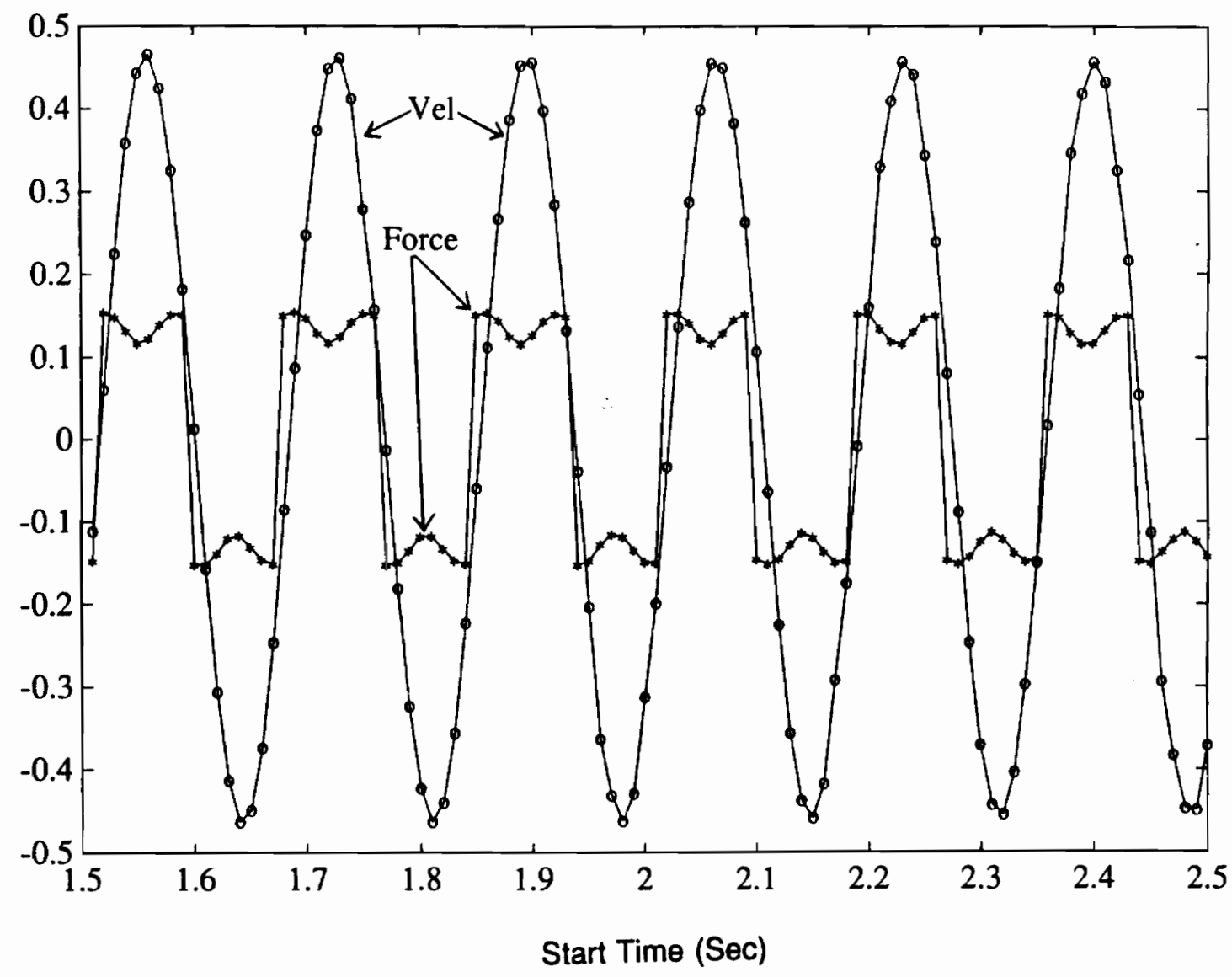

Figure 4-3

Analytical Maximum Force (lbs) and Initial Velocity (in/sec) for Suspended Mass Versus Start Time $(\alpha=1.5)$ 


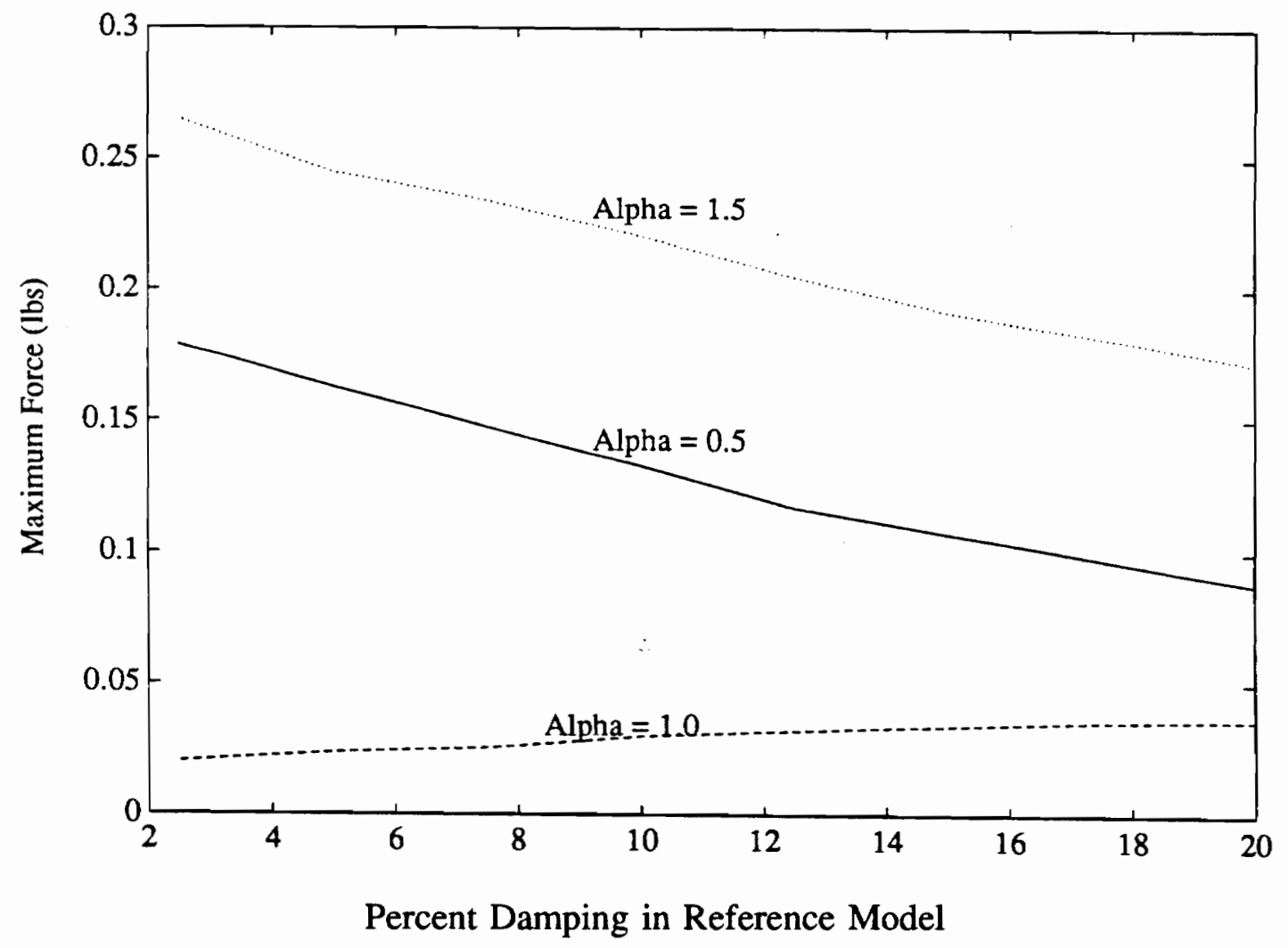

Figure 4-4

Effect of Damping on Maximum Force for Varying Amounts of Frequency Difference (Suspended Mass) 


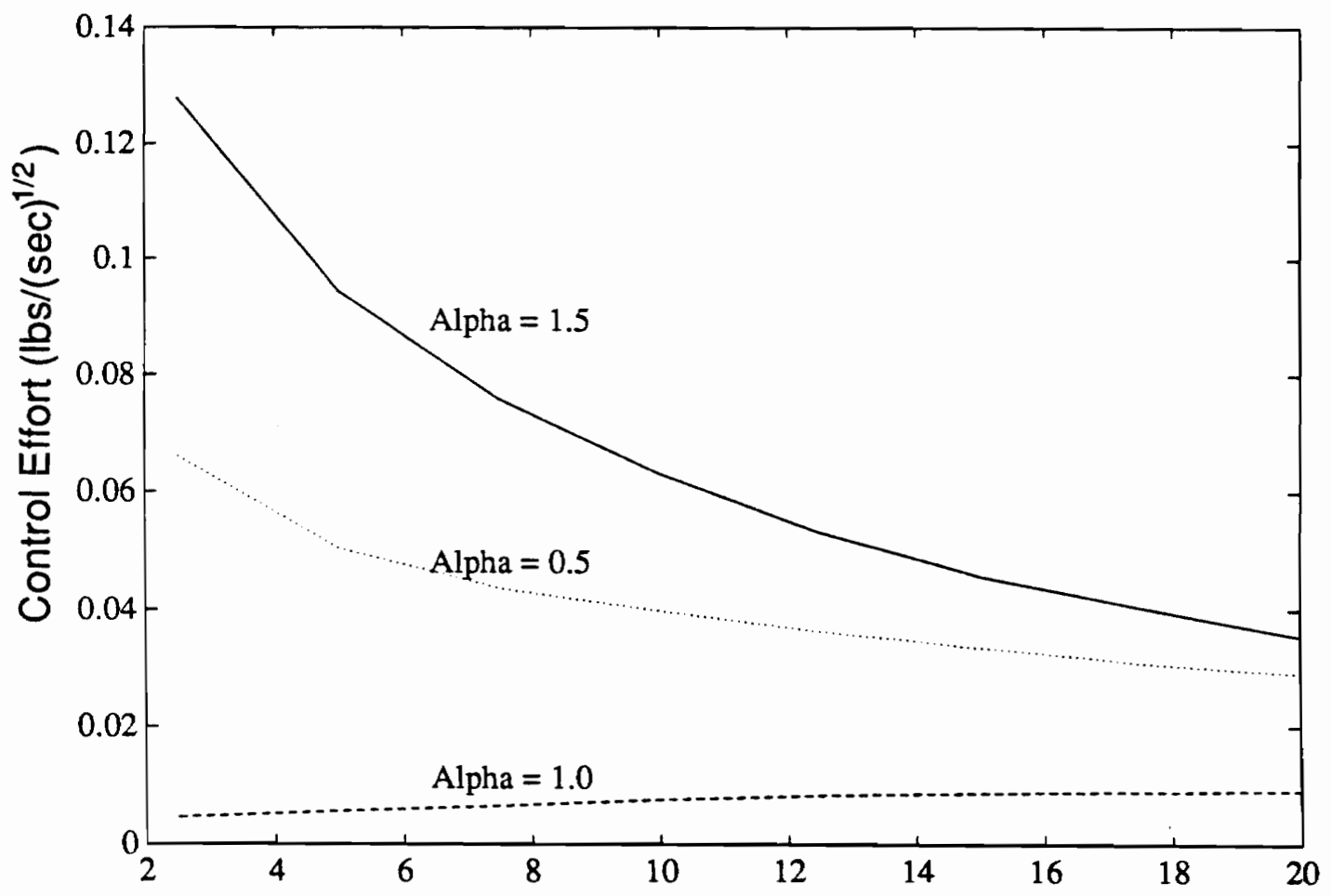

Percent Damping in Reference Model

Figure 4-5

Effect of Damping on Control Effort for

Varying Amounts of Frequency Difference (Suspended Mass) 


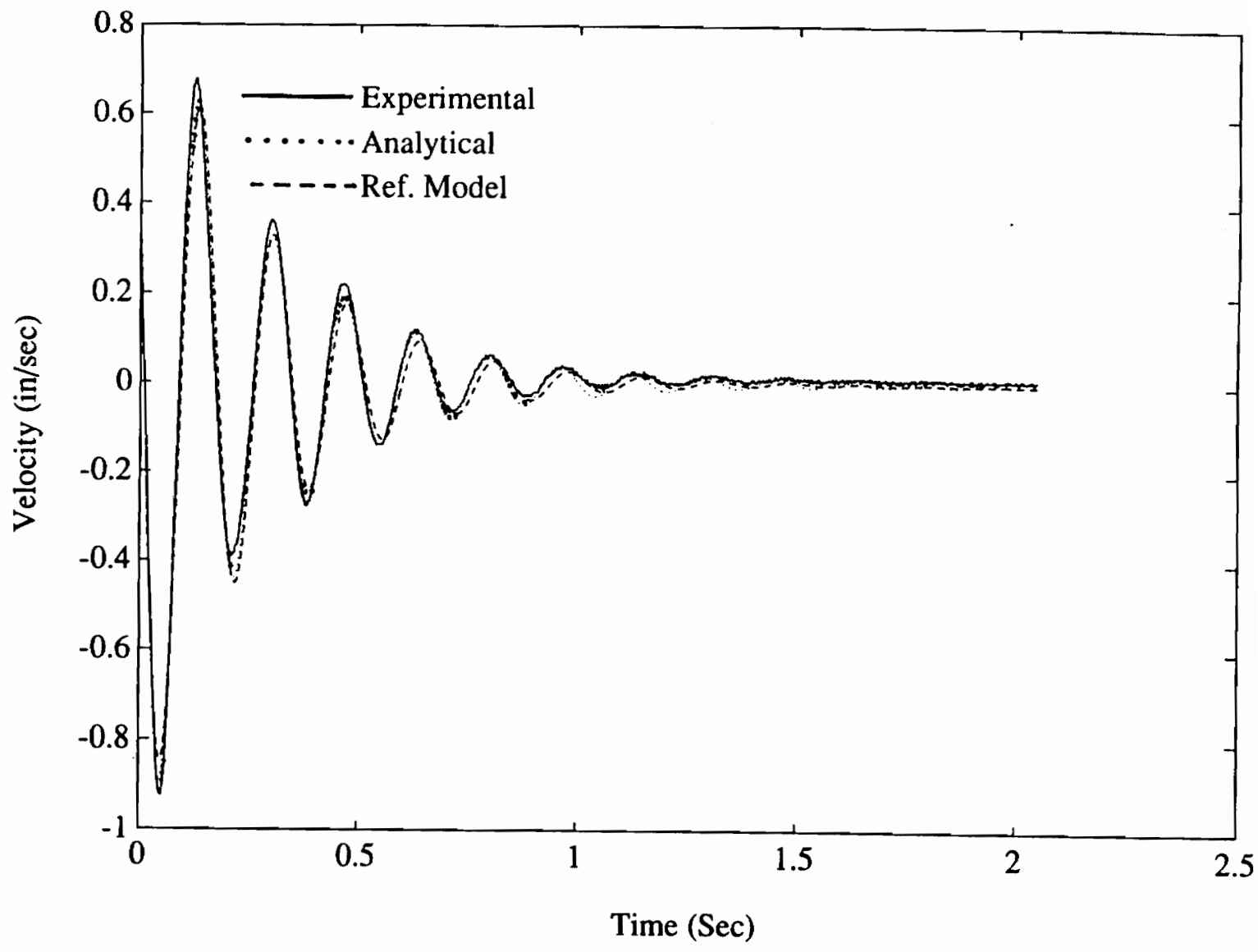

Figure 4-6

Comparison of Experimental and Analytical Velocity Response of the Suspended Mass System, $\omega_{\mathrm{nm}}=1.0 \omega_{\mathrm{np}}$ 


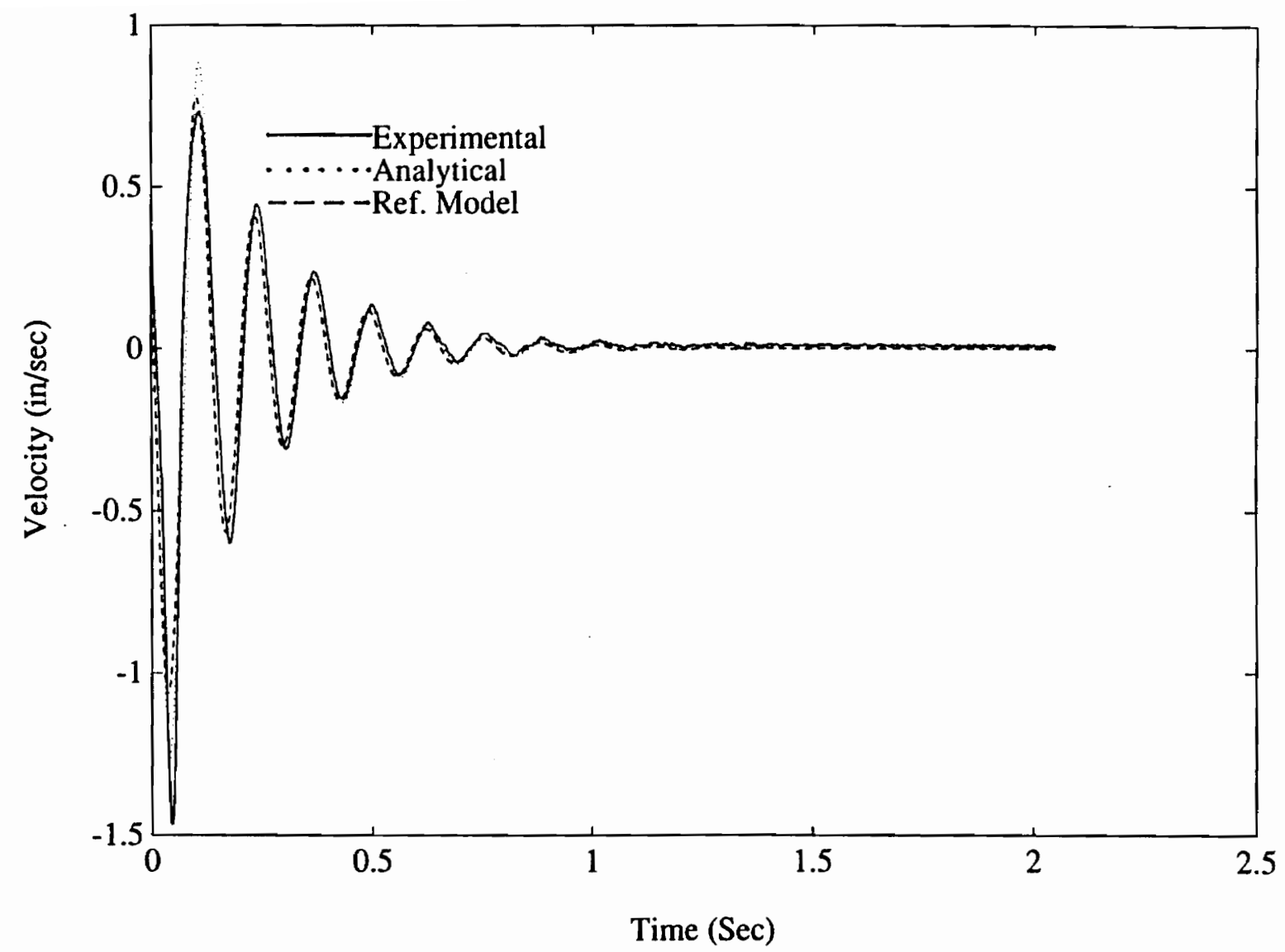

Figure 4-7

Comparison of Experimental and Analytical Velocity Response of the Suspended Mass System, $\omega_{\mathrm{mm}}=1.3 \omega_{\mathrm{np}}$ 


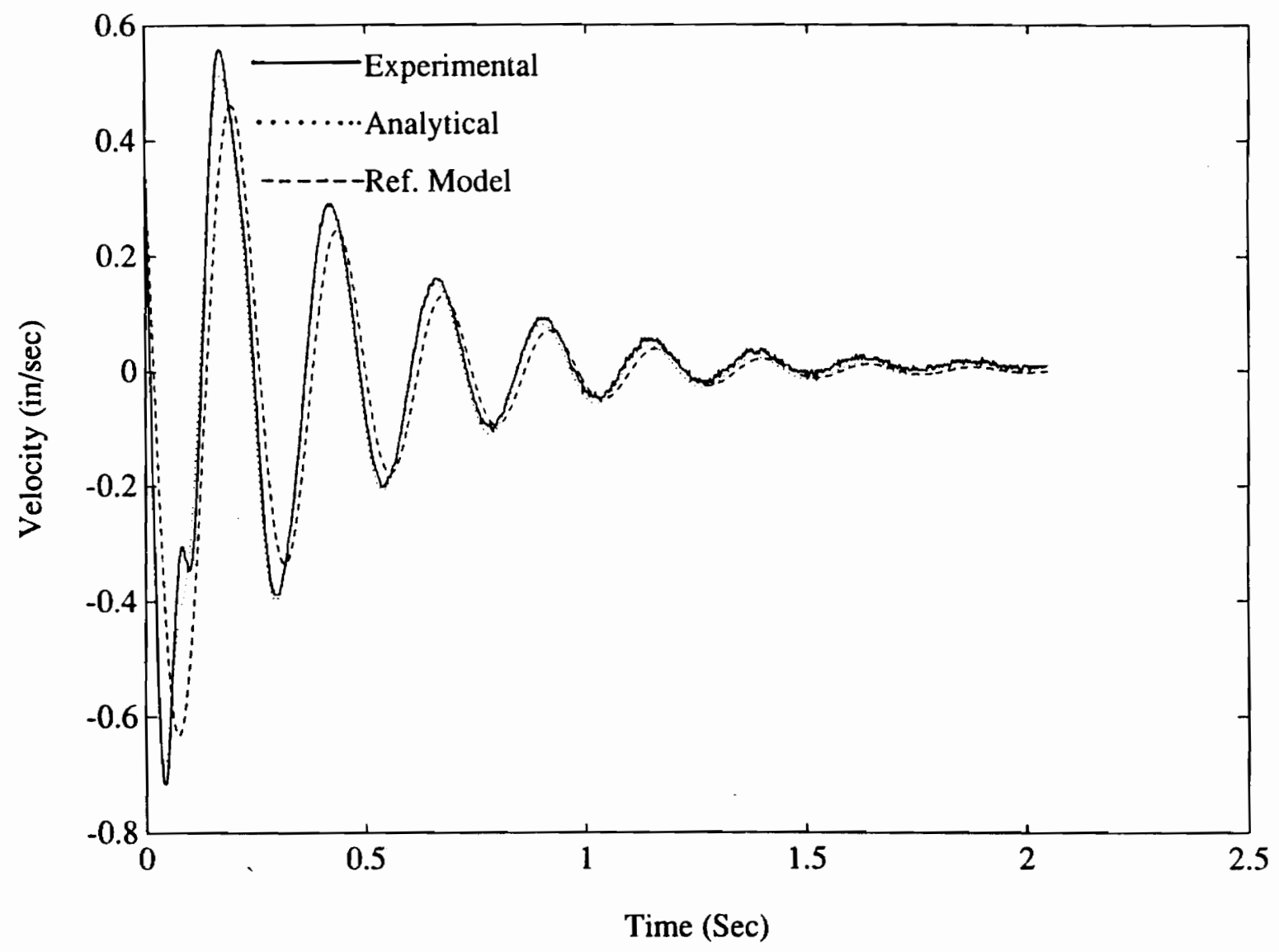

Figure 4-8

Comparison of Experimental and Analytical Velocity Response of the Suspended Mass System, $\omega_{n \mathrm{~m}}=0.7 \omega_{\mathrm{np}}$ 


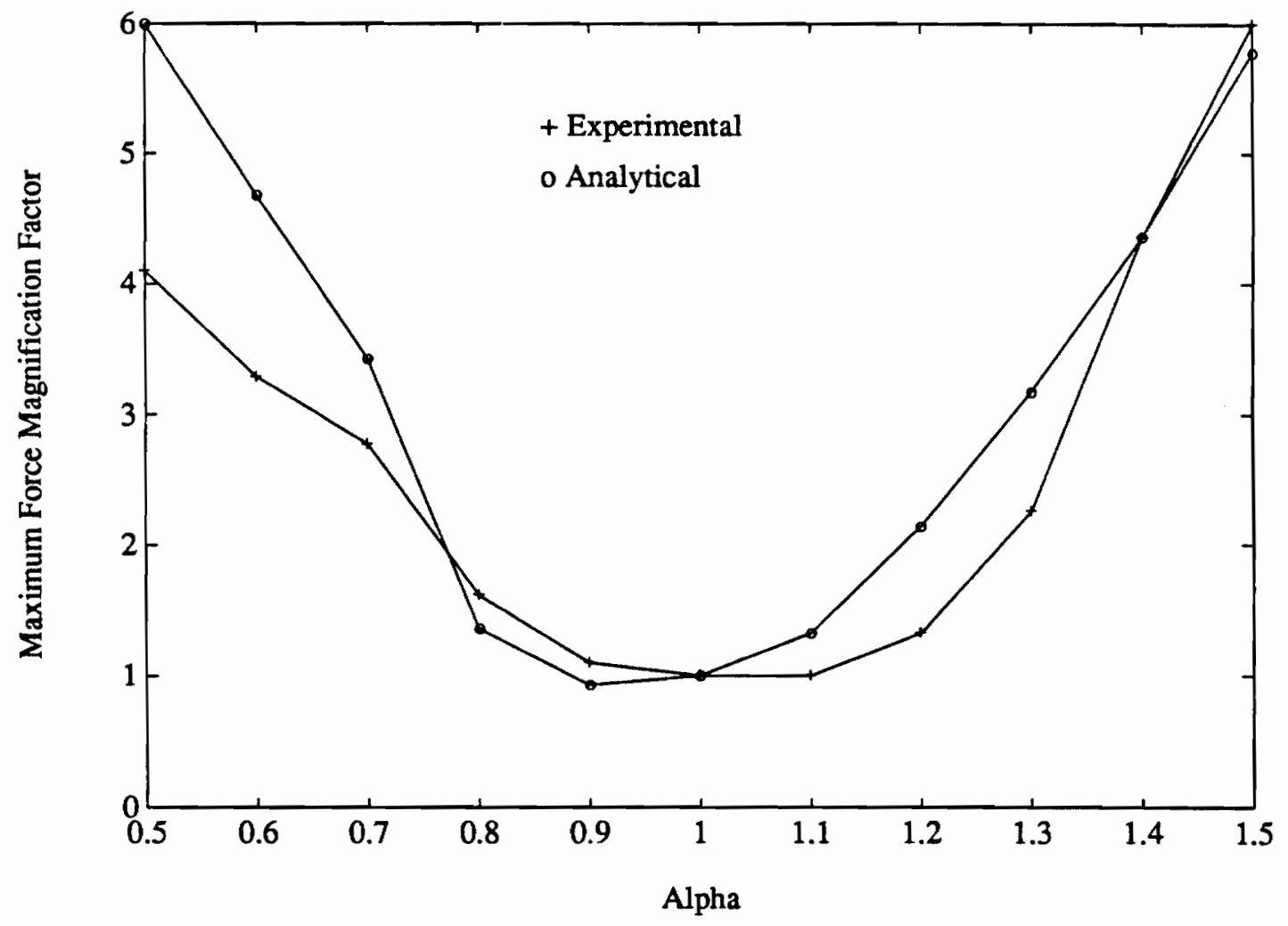

Figure 4-9

Comparison of Analytical and Experimental Maximum Force

Magnification Factor for the Suspended Mass 


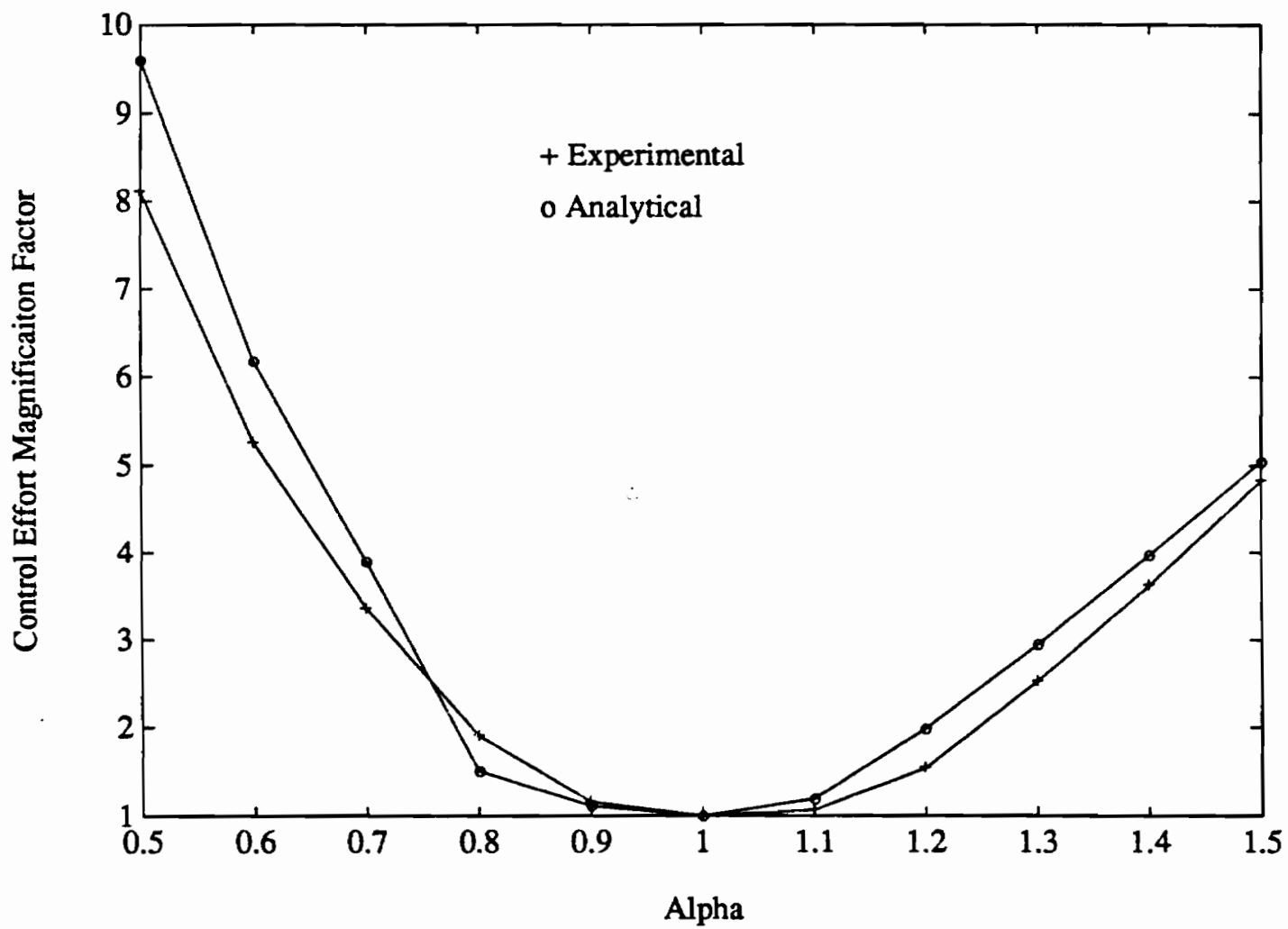

Figure 4-10

Comparison of Analytical and Experimental Control Effort Magnification Factor for the Suspended Mass 


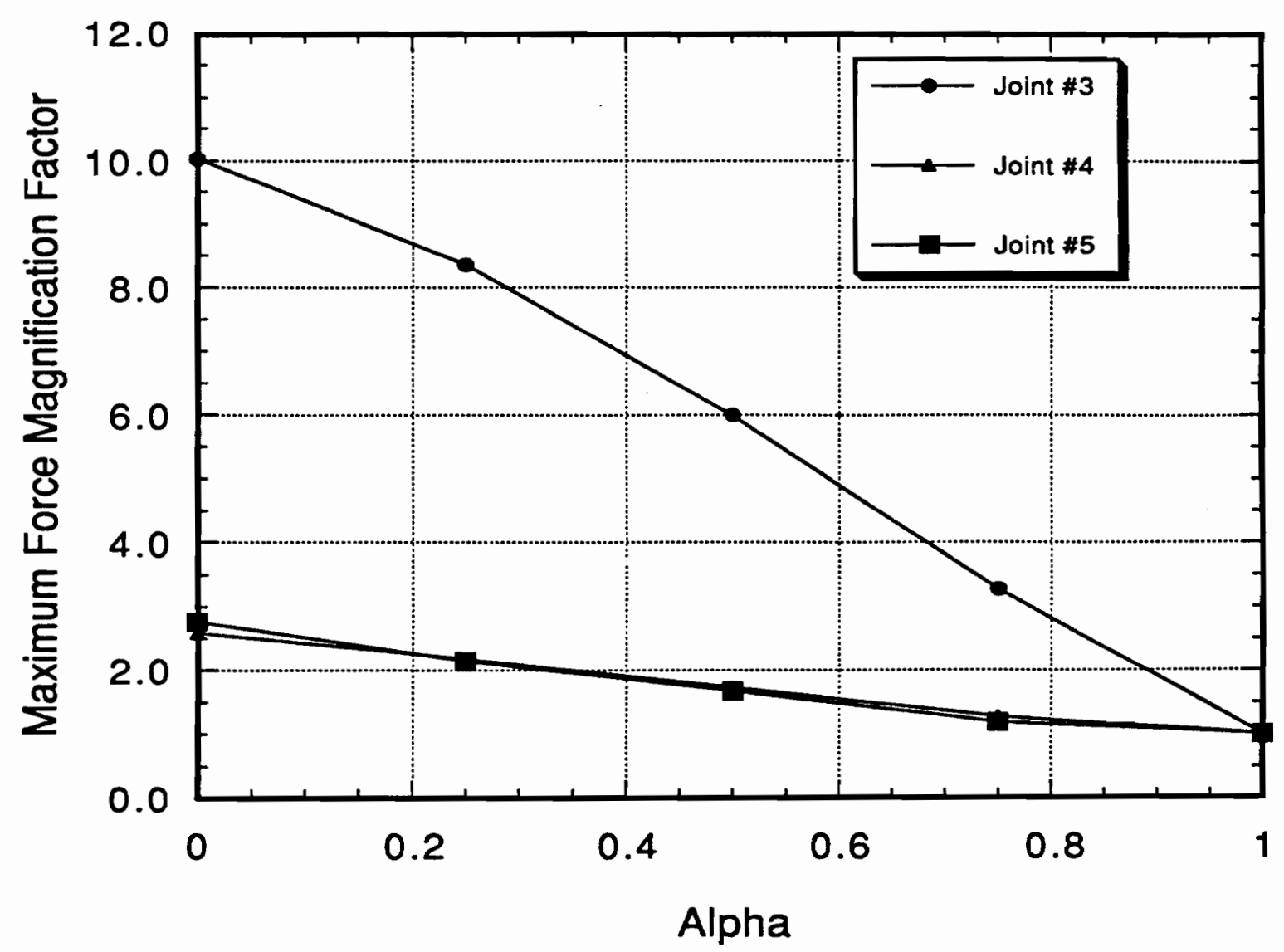

Figure 4-11

Analytical Maximum Force Magnification Factor

for the Slewing Grid (Initial Displacement = Analytical Second Mode) 


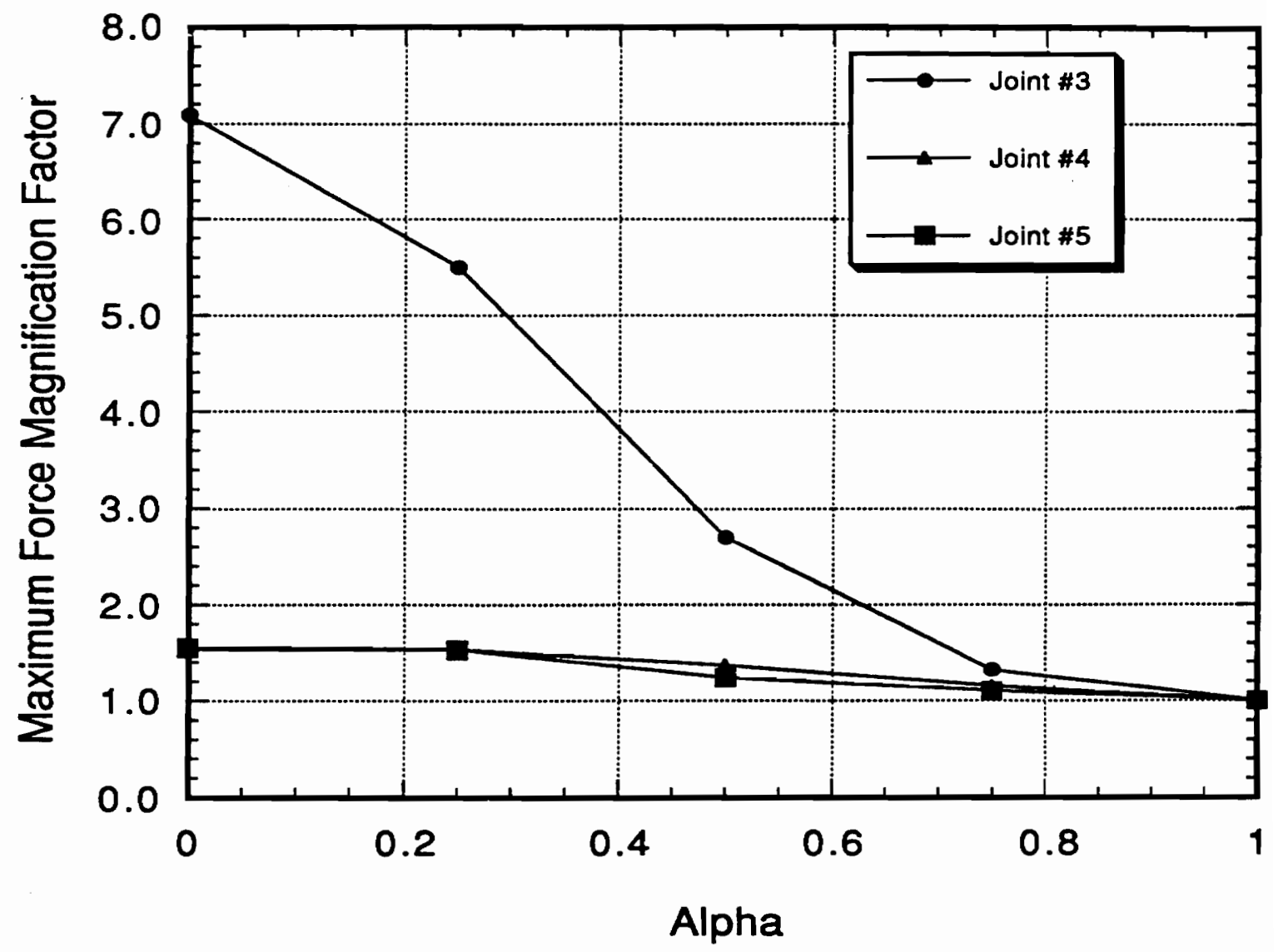

Figure 4-12

Analytical Maximum Force Magnification Factor

for the Slewing Grid (Initial Displacement is Analytical Fourth Mode) 


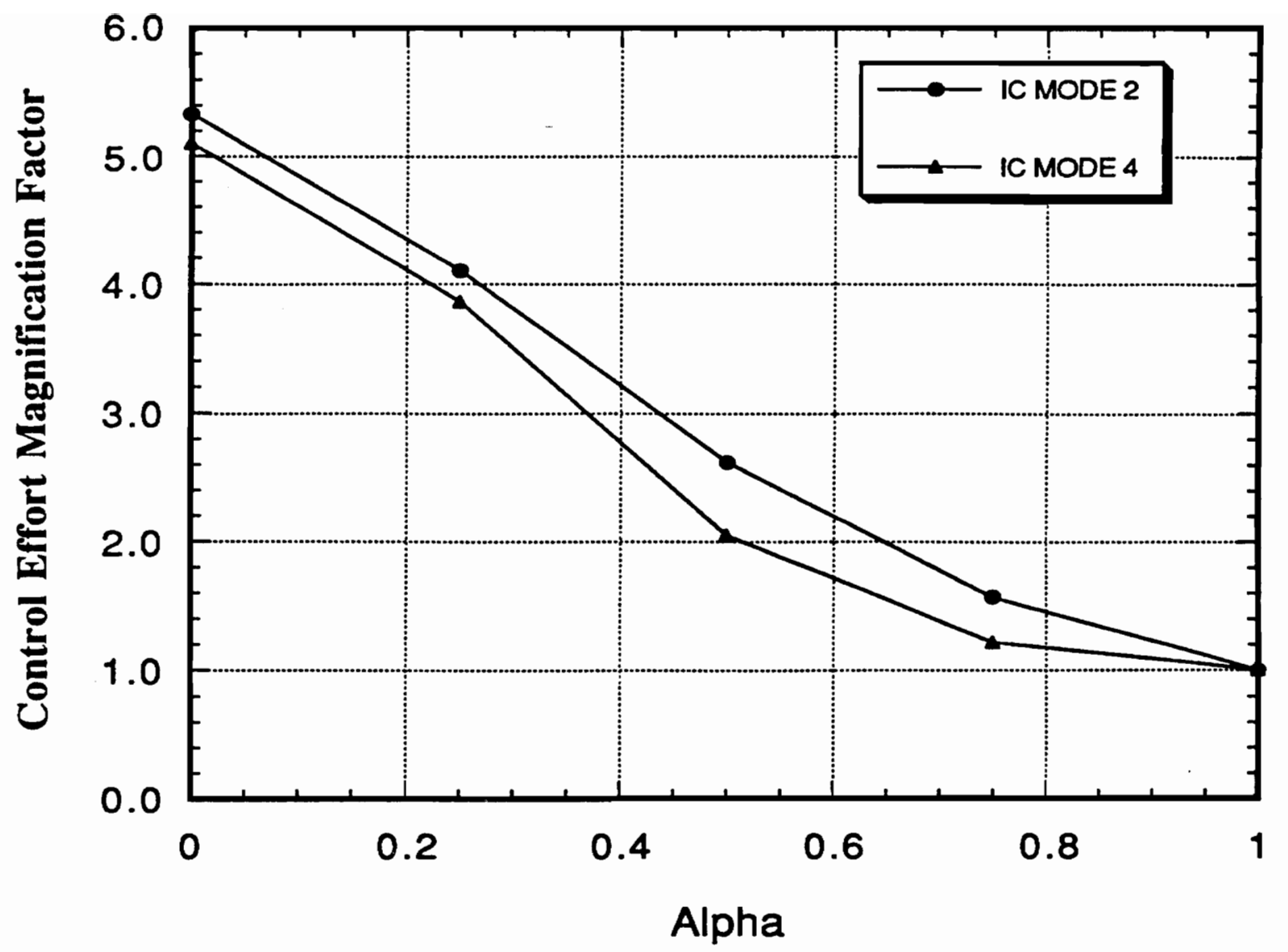

Figure 4-13

Analytical Control Effort Magnification Factor

for the Slewing Grid 


\section{Chapter 5 \\ Weighting Matrix Optimization}

As seen in Chapter 4, reasonable amounts of difference between the reference model and the actual plant can cause large increases in the magnitude of control effort and maximum force $U_{\text {pmax }}$ required to force the plant to follow the reference model. It is desirable to minimize the effect of these differences by varying any available parameters in the adaptive control law. The objective of this chapter is to show that significant reductions in maximum force can be achieved by optimizing the $T$ and $T^{*}$ matrices of the control law. This is demonstrated analytically and experimentally for the Suspended Mass system. However, improvements in maximum force are meaningful only if they are not at the expense of the performance of the MRAC in terms of following the reference model. So we begin this chapter with a discussion of a quantitative measure of MRAC performance.

\subsection{Measure of Fit for Suspended Mass System}

In order to maintain or improve plant following performance (fit), it was necessary to quantify how well the plant follows the reference model. In developing this fitness parameter we consider two types of discrepancies between the plant and the model. The first is a phase difference between the two systems (see fig. 5-1) and the second is the average amplitude difference. Therefore, the measure of fit $f$ was defined as

$$
f=\min _{d}\left[\int_{t_{0}}^{t_{f}}\left|v_{p}(t)-v_{m}\left(t+t_{d}\right)\right| d t\right]+\eta\left|t_{d}\right|
$$


where $t_{d}$ is a time delay selected to minimize the difference between the plant velocity $V_{p}$ and model reference velocity $V_{m}, t_{0}$ is the time when the controller is turned on, and $t_{f}$ is the time when the controller is turned off. It is critical that the time delay be used, because a small time shift can cause the difference between the two velocity signals to be very large while the fit is very good. For the Suspended Mass, $t_{d}$ was varied between -0.06 to 0.06 seconds, and $\eta$ was taken as $0.5 \mathrm{in} / \mathrm{sec}$. By minimizing the integral over

the time delay the two velocities are compared without being corrupted by the lead/lag time. Figures 5-1 through 5-4 show sample velocity traces used to determine the value of the fit $\mathrm{f}$. Figures 5-1 and 5-2 show the unshifted velocity traces for $\alpha=0.5$ and $\alpha=$ 1.5, respectively, and figures 5-3 and 5-4 show the same velocity traces with the time delay taken out. The values of $\mathrm{f}$ for figures 5-1 and 5-2, where the time shift is zero, are 0.116 in. and 0.054 in respectively. Whereas for figures 5-3 and 5-4, which include time shifts of $0.024 \mathrm{sec}$. for $\alpha=0.5$ and $0.008 \mathrm{sec}$. for $\alpha=1.5$, the corrected values of $\mathrm{f}$ are 0.06 in. for $\alpha=0.5$, and 0.04 for $\alpha .=1.5$.

\subsection{Choice of Design Variables}

From equations 2-6 to 2-10 it can be seen that the only parameters controlled by the designer are the $T$ and $T^{*}$ matrices. Furthermore, since $U_{m}$ is assumed to be zero, the fourth row and column of both $T$ and $T^{*}$ have no effect on the controller. Therefore only 9 elements of each matrix are specified by the designer. Figures 5-5 to 5-9 show how changes in the $T$ and $T^{*}$ matrices affect $F_{\max }$ and $f$ for $\alpha=1.0$, where the frequency difference between the plant and the reference model was zero. Figures 5-5 and 5-6 show that increasing the diagonal elements of the $T$ matrix from 1 to 100 causes $F_{\max }$ to approach $0.195 \mathrm{lbs}$. The fit is halved from $\mathrm{f}=0.12 \mathrm{in}$. at $\mathrm{T}=\mathrm{I}$ to $\mathrm{f}=0.059$ in. at $\mathrm{T}=16 \mathrm{I}$, then rises slowly up to $f=0.063$ in. at $T=45 I$, and finally decreases monotonically to $f=0.058$ in. at $\mathrm{T}=100 \mathrm{I}$. On a larger scale, figures 5-7 and 5-8 show that analytically, increasing the $T$ matrix to $T=1500 I$ improves $f$ by a factor of 6 while only increasing $F_{\max }$ by 2 . However, figure 5-9 shows the experimental results don't agree. Figure 5-9 shows that 
as $T$ increase, $F_{\max }$ increases almost linearly with no improvement in $\mathrm{f}$. These results are similar to those shown analytically by Bayard (ref. 40 ). In addition, at $T=450 I$ the system becomes unstable. Figures 5-5 and 5-6 also show that increasing $T^{*}$ also causes $F_{\max }$ and $f$ to get large except the range of acceptable values for $T^{*}$ are much smaller. For example, $T^{*}=35 I$ is the largest value of $T^{*}$ that can be used before $F_{\max }$ and $f$ get large without bound. It will be shown in section 5.5 that the value of $T^{*}$ at which the $F_{\max }$ gets large is a function of the sampling rate of the controller. For previous results, these matrices were chosen to be $T=50[I]$ and $T^{*}=[\Pi]$ and good performance and stability was achieved (see figures 5-1 \& 5-2).

\subsection{Optimization Formulation}

From the above discussion, the need to use optimization to choose the best combination of $\mathrm{T}^{\mathrm{and}} \mathrm{T}^{*}$ is clear. However, the use of optimization for MRAC is limited by the fact that the error in any real application is unknown. In the present application, the optimizer will use $\alpha=0.5$ and $\alpha=1.5$ for limits on the errors of the system. Considering that the reason for using MRAC is the lack of knowledge about the system, the best possible situation for most applications is to choose upper and lower bounds for errors in the system and then optimize for the chosen bounds. The problem is further complicated because the optimizer assumes that it knows the bounds on all differences between the reference model and the plant, when in reality, there will always be differences (such as model reduction) that the optimizer is not aware of. Therefore, before even beginning, the designer should realize that the optimal designs produced by the optimizer may eventually predict improvement beyond what the plant can actually realize. Even with the Suspended Mass system (the simplest possible structure), this phenomenon is observed. The optimizer is given accurate values for the upper and lower bounds on the frequency, but other differences which occur because of the digital implementation are neglected. As will be seen in section 5-4 the experimental and analytical correlation remains good for the initial optimal design, but 
experimental/analytical correlation is lost as the optimizer makes the optimal design better and better.

As stated above, the Suspended Mass system requires the optimal solution be effective for all values of $\alpha$. Figures 5-10 and 5-11 show that as we move away from the perfect knowledge case $(\alpha=1.0)$ to the maximum error cases $(\alpha=1.5$ and $\alpha=0.5), \mathrm{F}_{\max }$ and $f$ increase. We assume that the worst case will be either for $\alpha=0.5$ or for $\alpha=1.5$. Therefore the optimization problem is formulated as,

$$
\begin{gathered}
\text { Find } \mathrm{T}, \mathrm{T}^{*} \text {, and } \mathrm{F}_{\max } \text { to Minimize } \mathrm{F}_{\max }, \\
\text { Such That, } \\
g_{1}(x)=f_{\max }-f(\alpha=0.5) \geq 0, \\
g_{2}(x)=f_{\max }-f(\alpha=1.5) \geq 0, \\
g_{3}(x)=F_{\max }-U_{\max }(\alpha=0.5) \geq 0, \\
g_{4}(x)=F_{\max }-U_{\mathrm{pmax}}(\alpha=1.5) \geq 0 .
\end{gathered}
$$

The worst fit allowable is $f_{\max }$, and was chosen to be 0.06 inches after comparing figs. 5-1 to $5-4$, and $F_{\max }$ is the larger of $U_{p \max }(\alpha=0.5)$ and $U_{p \max }(\alpha=1.5)$. Note $F_{\max }$ is considered as both a design variable and the objective function. This approach is necessary because $\mathrm{F}_{\max }$ must take on the largest of two values, and is not a smooth function; including $\mathrm{F}_{\max }$ as a design variable avoids this problem. The optimizer (an IMSL subroutine called DNOONF) uses a sequential quadratic programming algorithm and a finite difference gradient (ref. 50). After some experimentation with step sizes, derivatives of the objective function and constraints were calculated with a forward difference step size of $10^{-4}$.

\subsection{Optimization Results Using Only Diagonal Elements}

As a start, we checked whether the design with scalar matrices $T=50 \mathrm{I}$ and $\mathrm{T}^{*}=\mathrm{I}$ 
obtained by a rough experimental optimization for $\alpha=1.0$, can be improved for the range $0.5 \leq \alpha \leq 1.5$. The matrices $T$ and $T^{*}$ were first defined as $T=\gamma_{1}[I]$ and $T^{*}=\gamma_{2}[I]$, with $\gamma_{1}$ and $\gamma_{2}$ as design variables. The initial values for the design variables were $\gamma_{1}=50.0$, and $\gamma_{2}=1.0$. Table 5-1, case 1 , shows the optimal design $\gamma_{1}=14.24$ and $\gamma_{2}=1.143$ and compares analytical values with experimental results. The optimizer reduced the analytical $\mathrm{F}_{\max }$ by $22 \%$, while at the same time improving the fit slightly. Experimental results show a $24 \%$ decrease in $F_{\max }$ while preserving $f(\alpha=0.5)=0.06$, and losing only a small amount of fit at $\alpha=1.5, \mathrm{f}(\alpha=1.5)=0.044$.

Case 2 allowed $T$, and $\mathrm{T}^{*}$ to be general diagonal matrices using the optimum of case 1 as the initial design. From table 5-1 it can be seen that the optimizer changed the diagonal elements of $T^{*}$, but neither $F_{\max }$ or $f$ changed by a significant amount. Experimental results also showed the same lack of improvement in $F_{\max }$ and $f$.

\subsection{Optimization Results Using Off Diagonal Elements}

The final step in the optimization process was a systematic optimization of the off diagonal elements to see which ones produced the most improvement. Starting with the optimal design of case 1, derivatives of the objective function with respect to each element in the $T$ and $T^{*}$ matrices were calculated to determine the largest derivatives. Table 5-2 shows that $T^{*}(1,2)$ and $T^{*}(2,1)$ have the largest derivatives, and thus were the best candidates for inclusion in the design variables list. Case 3 (table 5-3) includes all diagonal elements of $T$ and $T^{*}$, and off diagonal elements $T^{*}(1,2)$, and $T^{*}(2,1)$ as design variables. Starting from the analytical optimal design of case 1, table 5-4 shows that by adding these two off diagonal elements the optimizer can analytically reduce $F_{\max }$ by an additional $18 \%$ without increasing the fit parameters. However, experimentally this same design increases $F_{\max }(\alpha=1.5)$ by $65 \%$ from 0.13 lbs to 0.215 lbs but improves the fit ( $\alpha$ $=1.5$ ) by $15 \%$. To explore the reason for the divergence of analytical and experimental trends we changed the design gradually between case 2 and case 3 . The intermediate $T_{I}$ and $T_{I}^{*}$ are calculated by 


$$
\begin{gathered}
T_{I}=(1-\beta) T_{2}+\beta T_{3} \\
T^{*}{ }_{I}=(1-\beta) T^{*}{ }_{2}+\beta T^{*}{ }_{3}
\end{gathered}
$$

where the subscripts 2 and 3 refer to the optimal designs of cases 2 and 3 respectively. The variations of the maximum force and the fit as a function of $\beta$ are shown in figures 5-12 and 5-13. Figure 5-12 shows that $F_{\max }$ analytical and $F_{\max }$ experimental immediately begin to diverge no matter how small the $\beta$ is. However, figure 5-13 shows that the analytical and experimental fit both show an improvement trend. After some searching for the cause of the discrepancy we identified the sampling rate as a major contributor. We increased the sampling rate of the DSP board from $500 \mathrm{~Hz}(0.002 \mathrm{sec} . / \mathrm{cycle})$ to 1000 $\mathrm{Hz}(0.001 \mathrm{sec} . / \mathrm{cycle})$ and finally to $2000 \mathrm{~Hz}(0.0005 \mathrm{sec} . / \mathrm{cycle})$. Figures 5-14 and 5-15 show maximum force results, at the three sampling rates, for several designs which include increasingly larger off diagonal elements. For $\beta=0$, the $T$ and $T^{*}$ matrices are the optimal design of case 2 . As $\beta$ increases from 0 to 1 the $T^{*}(1,2)$ and $T^{*}(2,1)$ elements are increased linearly until at $\beta=1, T^{*}(1,2)=T^{*}(2,1)=-1.408$. By changing only these two elements, we were able to isolate the effect of adding off diagonal elements.

From figure 5-14 it can be seen that for $\alpha=1.5$ the value of $\beta$ where the analytical and experimental $U_{\text {pmax }}$ begin to diverge increases as the sampling rate gets higher. For a sampling rate of $500 \mathrm{~Hz}$ the experimental trends follow the analytical trends only for $\beta \leq 0.1$, and the maximum force is smaller than the $\beta=0$ case only for $\beta \leq 0.35$. For the $2000 \mathrm{~Hz}$ case experimental trends follow analytical trends for $\beta \leq 0.2$, and the maximum force is less than the $\beta=0$ case for $\beta \leq 0.5$. It is also important to note that for the $\beta=0$ (i.e. no off diagonal terms) the value of $U_{p \max }$ is decreased by almost $10 \%$ by increasing the sampling rate from $500 \mathrm{~Hz}$ to $2000 \mathrm{~Hz}$. This is an interesting result because $500 \mathrm{~Hz}$ sampling is well above the traditional rule of 10 times the frequency to be controlled. Figure 5-15 shows similar trends for $U_{p \max }$ at $\alpha=0.5$, except that the values of $\beta$ where the experimental and analytical values begin to diverge is twice the values for $\alpha=1.5$, and the improvement in experimental maximum force at $\beta=0$ is very 
small. Figure 5-16 shows similar trends for the fit parameter at $\alpha=1.5$. Improvement is shown not only in the trends but also in the value of the fit. For the $500 \mathrm{~Hz}$ case, the trends of the experimental fit parameter do not resemble the analytical trends, but the experimental trends of the $2000 \mathrm{~Hz}$ case follow the analytical trends closely.

The improvement in the value of fit may be misleading because the range over which it is calculated decreases as the sampling rate increases. Because of hardware limitations the length of the velocity vectors used to calculate the experimental fit had to be held constant. Therefore the fit calculation for the $500 \mathrm{~Hz}$ case cover a 2 second range, while the fit calculation for the $2000 \mathrm{~Hz}$ case use only a 0.5 second range. Although this decrease in the range used for the experimental fit calculation changes the magnitude at $\alpha=1.5$, the trends should be similar since the system is about two thirds damped out after 0.5 seconds (compare damping for $\alpha=1.3$, figure 4-7). However, for $\alpha=0.5$ the system has only completed $1 \frac{1}{2}$ cycles in 0.5 seconds, and no observation about the effects of increasing the sampling rate can be made.

In figures 5-14 through 5-16, it is observed that almost all the improvement between experimental and analytical correlation came as a result of improvement in the experimental results. This suggests that the off-diagonal elements introduce some type of high frequency motion or control transients into the system which the analysis does not model. However, from fig. 5-14 it can also be seen that for $\beta=0$ (zero on the offdiagonal elements) changing the sampling rate from $500 \mathrm{~Hz}$ to $1000 \mathrm{~Hz}$ reduced $F_{\max }$ by $8.4 \%$. This is significant reduction since $500 \mathrm{~Hz}$ was already 41 time the Nyquist frequency. The importance of the sampling rate can also be seen from fig. 5-17, where the maximum force as a function of $\mathrm{T}^{*}$ is plotted for a sampling rate of $2000 \mathrm{~Hz}$. Comparing fig. 5-17 with fig. 5-5 it is seen that the point at which $F_{\max }$ gets very large, can be postponed by increasing the sampling rate. In fig. 5-5 it is shown that at $500 \mathrm{~Hz}$ $\mathrm{T}^{*}=35 \mathrm{I}$ is the largest value of $\mathrm{T}^{*}$ that can be used before $\mathrm{F}_{\max }$ gets large, whereas at 2000 Hz. (fig. 5-17) $\mathrm{T}^{*}=110 \mathrm{I}$ is the largest stable value.

It is clear that increasing the sampling rate of the DSP board is one way of improving 
the performance of the controller. However, the rates required to get accurate results may be very high, and at some point the time lag introduced into the system by digital implementation will become significant compared to the sampling rate, and increasing the sampling rate will not benefit the performance. For example, efforts to push the sampling rate for the Suspended Mass to $3000 \mathrm{~Hz}$ were stymied by high frequency instabilities. It's not clear whether these problems were caused by the time lag of the system, or the inability of the DSP board to perform the necessary calculations fast enough.

\subsection{Optimization Results Using Diagonal Elements and Damping Ratios}

Because of the effects that increased damping had on the maximum force as the frequency difference between the reference model and the plant increased (section 4.1), it was desirable to include the damping ratio of the reference model as a design variable. The purpose of this optimization was to see what the optimal value of damping would be, and to see if experimental and analytical trends would be the same. Figures 5-18 and 519 show experimental and analytical results for the maximum force as a function of the required damping in the reference model. Figure 5-18 shows that for $\alpha=0.5$, both experimental and analytical results show the maximum force decreasing as the damping is increased, and figure 5-19 show the same results for the $\alpha=1.5$ case. In section 4.1 this result was shown experimentally for the baseline design, but figures 5-18 and 5-19 also indicate that the experimental and analytical correlation is maintained as $T$ and $T^{*}$ are optimized, and thus damping could be included in the optimization without loss of experimental and analytical correlation. The optimizer was allowed to vary the damping ratio between $0 \%$ and $100 \%$. As might be expected, from figures 5-18 and 5-19, the optimal damping value was $100 \%$. This result indicates that it is easier for the controller to damp the system quickly than to make it have a different natural frequency. However, recalling that the effects of increased damping in the Slewing Grid were not so dramatic, it is not clear what optimal values of damping the optimizer may find if allowed to optimize the Slewing Grid design. Nevertheless, it seems safe to assume that the 
optimizer could improve the analytical design without giving up good experimental and analytical correlation. 
Table 5-1

Initial and Optimal Designs of The Diagonal elements

For the T and $\mathbf{T}^{*}$ Matrices (Suspended Mass System)

\begin{tabular}{|c|c|c|c|c|}
\hline \multirow{2}{*}{$\begin{array}{c}\text { Possible } \\
\text { Design } \\
\text { Variables and } \\
\text { Performance } \\
\text { Measures }\end{array}$} & \multicolumn{2}{|c|}{$\begin{array}{l}\text { Case } 1 \\
\text { Scalar Matrices }\end{array}$} & \multicolumn{2}{|c|}{$\begin{array}{c}\text { Case } 2 \\
\text { Diagonal Matrices }\end{array}$} \\
\hline & $\begin{array}{l}\text { Initial } \\
\text { Design }\end{array}$ & $\begin{array}{r}\text { Optimal } \\
\text { Design }\end{array}$ & $\begin{array}{l}\text { Initial } \\
\text { Design }\end{array}$ & $\begin{array}{r}\text { Optimal } \\
\text { Design }\end{array}$ \\
\hline$\gamma_{1}$ & $50.0^{\circledR}$ & 14.24 & 1.0 & 1.0 \\
\hline$\gamma_{2}$ & $1.0^{@}$ & 1.143 & 1.0 & 1.0 \\
\hline $\mathrm{T}(1,1)$ & 1.0 & 1.0 & $14.244^{@}$ & 14.244 \\
\hline $\mathrm{T}(2,2)$ & 1.0 & 1.0 & $14.244^{@}$ & 14.244 \\
\hline $\mathrm{T}(3,3)$ & 1.0 & 1.0 & $14.244^{@}$ & 14.244 \\
\hline $\mathrm{T}^{*}(1,1)$ & 1.0 & 1.0 & $1.143^{@}$ & 0.996 \\
\hline $\mathrm{T}^{*}(2,2)$ & 1.0 & 1.0 & $1.143^{@}$ & 1.192 \\
\hline$T^{*}(3,3)$ & 1.0 & 1.0 & $1.143^{@}$ & 1.144 \\
\hline$F_{\max }^{e}(\alpha=0.5)$ & $0.129^{e}$ & $0.100^{e}$ & $0.100^{e}$ & $0.100^{\mathrm{e}}$ \\
\hline $\mathrm{F}_{\max }(\alpha=0.5)$ & 0.161 & 0.126 & 0.126 & 0.126 \\
\hline $\mathrm{f}^{\mathrm{e}}(\alpha=0.5)$ & $0.061^{e}$ & $0.060^{e}$ & $0.060^{e}$ & $0.060^{e}$ \\
\hline$f(\alpha=0.5)$ & 0.064 & 0.060 & 0.060 & 0.060 \\
\hline $\mathrm{F}_{\max }^{\mathrm{e}}(\alpha=1.5)$ & $0.185^{\mathrm{e}}$ & $0.140^{\mathrm{e}}$ & $0.140^{\mathrm{e}}$ & $0.140^{e}$ \\
\hline $\mathrm{F}_{\max }(\alpha=1.5)$ & 0.151 & 0.128 & 0.128 & 0.128 \\
\hline $\mathrm{f}^{\mathrm{e}}(\alpha=1.5)$ & $0.032^{\mathrm{e}}$ & $0.044^{\mathrm{e}}$ & $0.044^{e}$ & $0.044^{\mathrm{e}}$ \\
\hline$f(\alpha=1.5)$ & 0.028 & 0.027 & 0.027 & 0.027 \\
\hline
\end{tabular}

@ Indicates variable used as a design variable in the optimization e Indicates the value was experimentally obtained 
Table 5-2

Derivatives of the Maximum Force With Respect to Off Diagonal Terms of the $T$ and $T^{*}$ Matrices, Evaluated at the Optimal Diagonal Design

\begin{tabular}{|c|c|c|}
\hline Off Diagonal Terms & $\partial F_{\max }(\alpha=0.5) / \partial \mathbf{X}_{\mathbf{i}}$ & $\partial \mathbf{F}_{\max }(\alpha=1.5) / \partial \mathbf{X}_{\mathbf{i}}$ \\
\hline$T(1,2)$ & $-0.1618 \mathrm{E}-2$ & $0.9172 \mathrm{E}-3$ \\
\hline$T(1,3)$ & $0.6836 \mathrm{E}-4$ & $-0.1155 \mathrm{E}-4$ \\
\hline$T(2,1)$ & $-0.2872 \mathrm{E}-3$ & $0.1274 \mathrm{E}-3$ \\
\hline$T(2,3)$ & $0.2994 \mathrm{E}-4$ & $-0.4675 \mathrm{E}-6$ \\
\hline$T(3,1)$ & $0.2212 \mathrm{E}-4$ & $-0.1651 \mathrm{E}-4$ \\
\hline$T(3,2)$ & $-0.7510 \mathrm{E}-4$ & $-0.2230 \mathrm{E}-4$ \\
\hline$T^{*}(1,2)$ & $-0.9735 \mathrm{E}-2$ & $0.3031 \mathrm{E}-1$ \\
\hline$T^{*}(1,3)$ & $0.5308 \mathrm{E}-3$ & $0.9703 \mathrm{E}-4$ \\
\hline$T^{*}(2,1)$ & $-0.9735 \mathrm{E}-2$ & $0.3031 \mathrm{E}-1$ \\
\hline$T^{*}(2,3)$ & $-0.5190 \mathrm{E}-3$ & $-0.4555 \mathrm{E}-3$ \\
\hline$T^{*}(3,1)$ & $0.5308 \mathrm{E}-3$ & $0.9703 \mathrm{E}-4$ \\
\hline$T^{*}(3,2)$ & $-0.5190 \mathrm{E}-3$ & $-0.4555 \mathrm{E}-3$ \\
\hline
\end{tabular}


Table 5-3

Initial and Optimal Designs with off Diagonal Elements of The $T$ and $\mathbf{T}^{*}$ Matrices (Suspended Mass System)

\begin{tabular}{|c|c|c|}
\hline \multirow{2}{*}{$\begin{array}{c}\text { Possible } \\
\text { Design } \\
\text { Variables }\end{array}$} & \multicolumn{2}{|c|}{ Case 3} \\
\hline & $\begin{array}{l}\text { Initial } \\
\text { Design }\end{array}$ & $\begin{array}{c}\text { Optimal } \\
\text { Design }\end{array}$ \\
\hline$\gamma_{1}$ & 1.00 & 1.00 \\
\hline$\gamma_{2}$ & 1.00 & 1.00 \\
\hline $\mathrm{T}(1,1)$ & $14.244^{@}$ & 22.727 \\
\hline $\mathrm{T}(1,2)$ & 0.0 & 0.0 \\
\hline $\mathrm{T}(1,3)$ & 0.0 & 0.0 \\
\hline $\mathrm{T}(2,1)$ & 0.0 & 0.0 \\
\hline $\mathrm{T}(2,2)$ & $14.244^{@}$ & 8.710 \\
\hline $\mathrm{T}(2,3)$ & 0.0 & 0.0 \\
\hline $\mathrm{T}(3,1)$ & 0.0 & 0.0 \\
\hline $\mathrm{T}(3,2)$ & 0.0 & 0.0 \\
\hline $\mathrm{T}(3,3)$ & $14.244^{@}$ & 14.284 \\
\hline$T^{*}(1,1)$ & $0.996^{@}$ & 1.767 \\
\hline$T^{*}(1,2)$ & $0.0^{@}$ & -1.373 \\
\hline$T^{*}(1,3)$ & 0.0 & 0.0 \\
\hline$T^{*}(2,1)$ & $0.0^{@}$ & -1.373 \\
\hline $\mathrm{T}^{*}(2,2)$ & $1.192^{@}$ & 1.725 \\
\hline $\mathrm{T}^{*}(2,3)$ & 0.0 & 0.0 \\
\hline$T^{*}(3,1)$ & 0.0 & 0.0 \\
\hline $\mathrm{T}^{*}(3,2)$ & 0.0 & 0.0 \\
\hline$T^{*}(3,3)$ & $1.144^{@}$ & 1.265 \\
\hline
\end{tabular}

@ Indicates variable was use as a design variable in the optimization 
Table 5-4

Experimental/Analytical Comparison of The

Maximum Force and Fitness (Suspended Mass System, $\alpha=0.5$ )

\begin{tabular}{|c|c|c|c|c|c|c|c|c|}
\hline \multirow{3}{*}{ Case } & \multicolumn{4}{|c|}{ Experimental } & \multicolumn{4}{|c|}{ Analytical } \\
\hline & \multicolumn{2}{|c|}{$\mathrm{F}_{\max }(\mathrm{lbs}) \times 10^{-3}$} & \multicolumn{2}{|c|}{ Fit(in.) $\times 10^{-3}$} & \multicolumn{2}{|c|}{$\mathrm{F}_{\max }(\mathrm{lbs}) \times 10^{-3}$} & \multicolumn{2}{|c|}{ Fit(in.) $\times 10^{-3}$} \\
\hline & $\alpha=0.5$ & $\alpha=1.5$ & $\alpha=0.5$ & $\alpha=1.5$ & $\alpha=0.5$ & $\alpha=1.5$ & $\alpha=0.5$ & $\alpha=1.5$ \\
\hline Baseline & 129 & 185 & 61 & 32 & 161 & 151 & 64 & 28 \\
\hline 1 & 100 & 140 & 60 & 44 & 126 & 128 & 60 & 27 \\
\hline 2 & 100 & 140 & 60 & 44 & 126 & 128 & 60 & 27 \\
\hline 3 & 92 & 191 & 60 & 39 & 104 & 105 & 60 & 18 \\
\hline
\end{tabular}




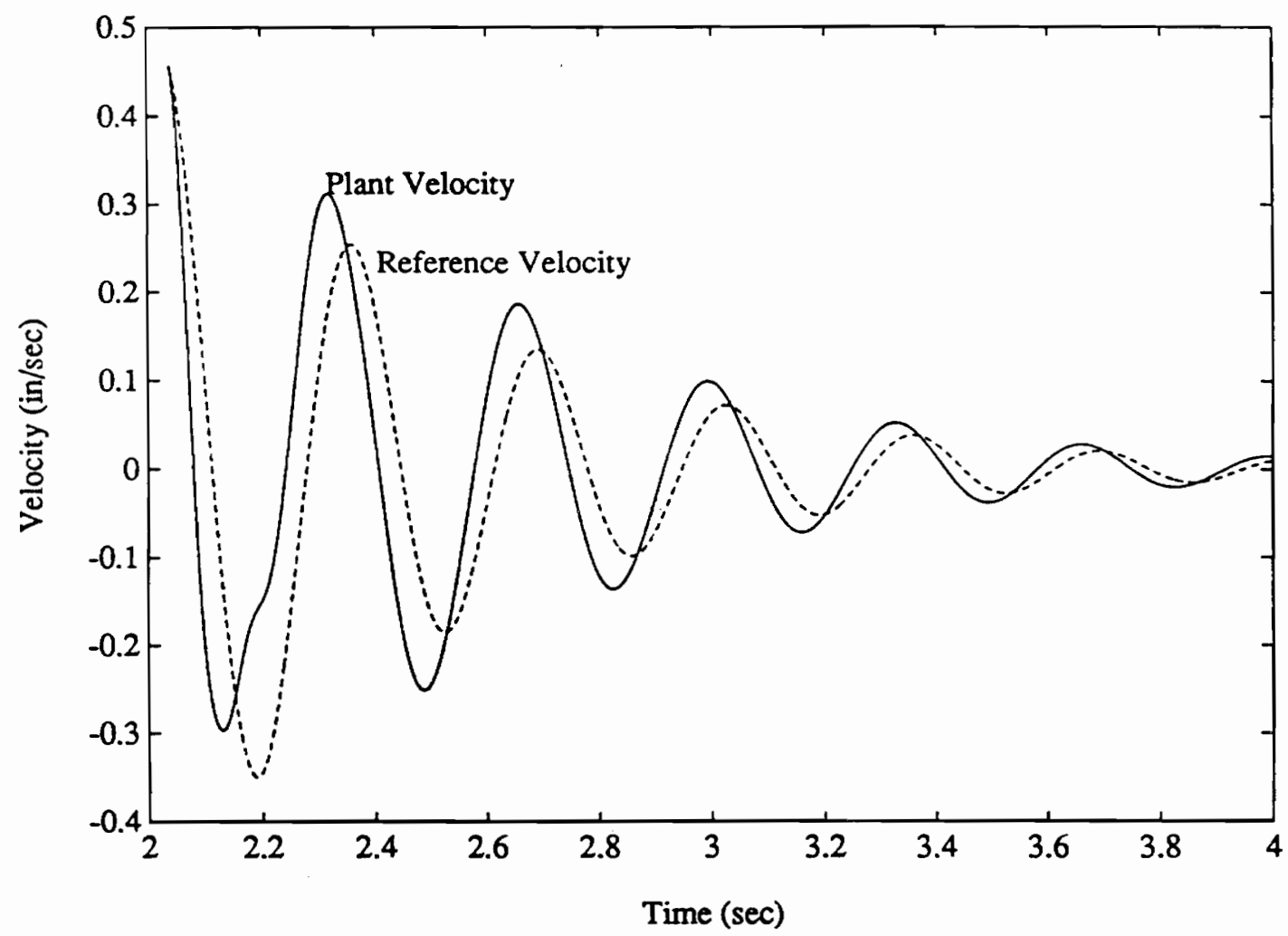

Figure 5-1

Unshifted Analytical Velocity Response of the Suspended Mass System $\left(\alpha=0.5, T=50.0[\mathrm{I}]\right.$, and $\left.\mathrm{T}^{*}=[\mathrm{II}]\right)$ 


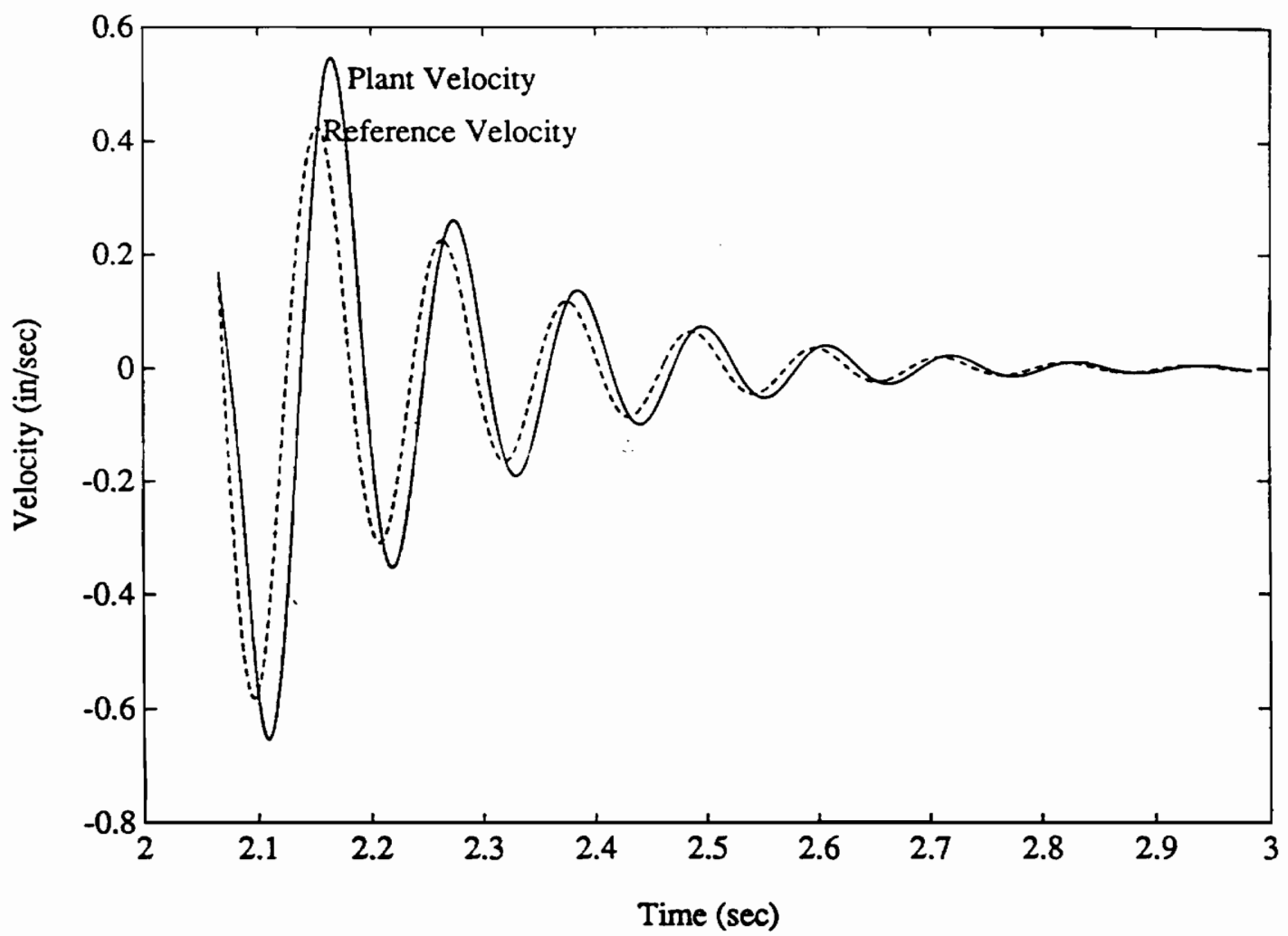

Figure 5-2

Unshifted Analytical Velocity Response of the Suspended Mass System $\left(\alpha=1.5, T=50.0[I]\right.$, and $\left.T^{*}=[I]\right)$ 


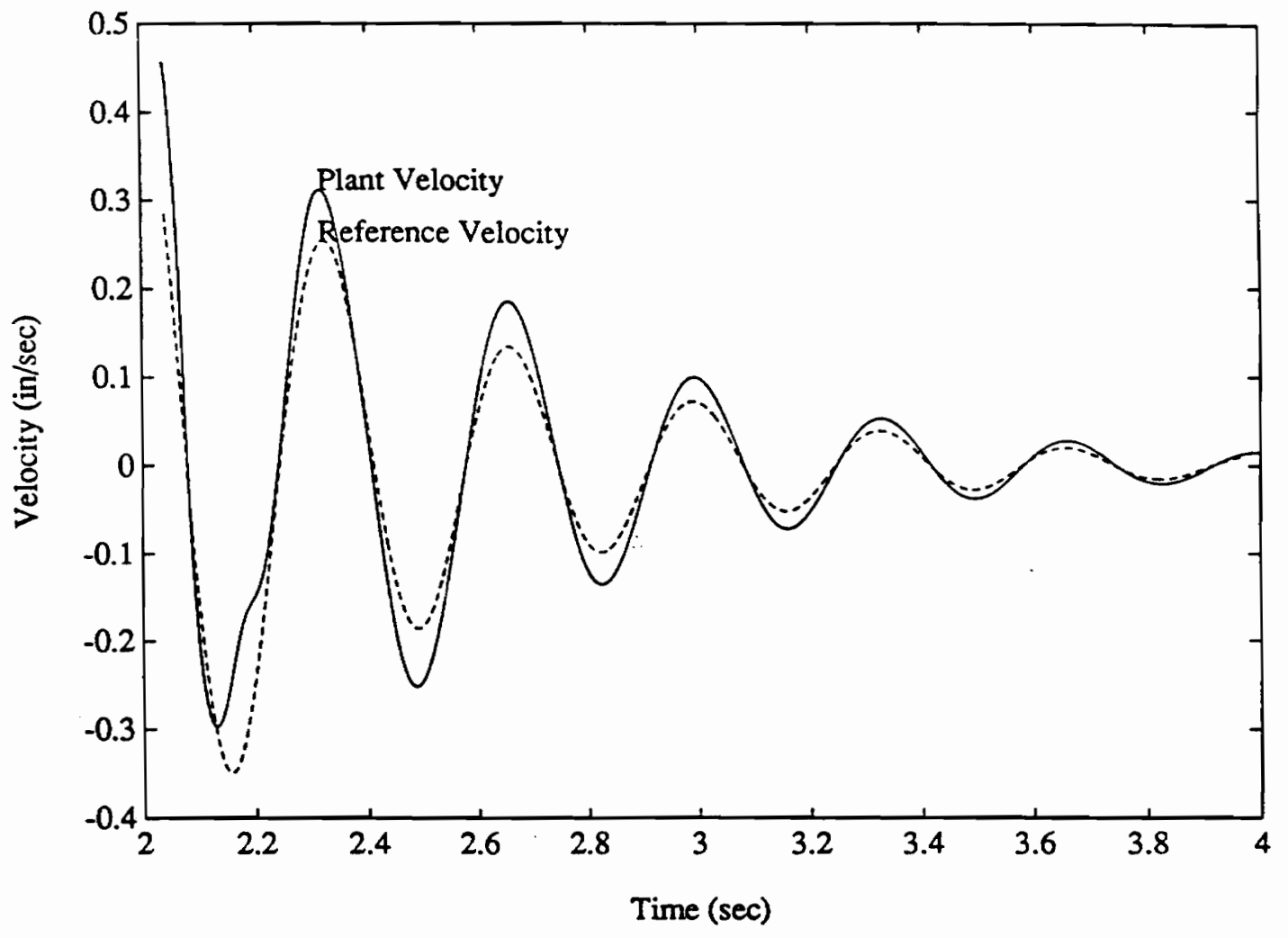

Figure 5-3

Shifted $\left(t_{d}=0.03\right.$ sec) Analytical Velocity Response of the Suspended Mass System $\left(\alpha=0.5, T=50.0[I]\right.$, and $\left.T^{*}=[I]\right)$ 


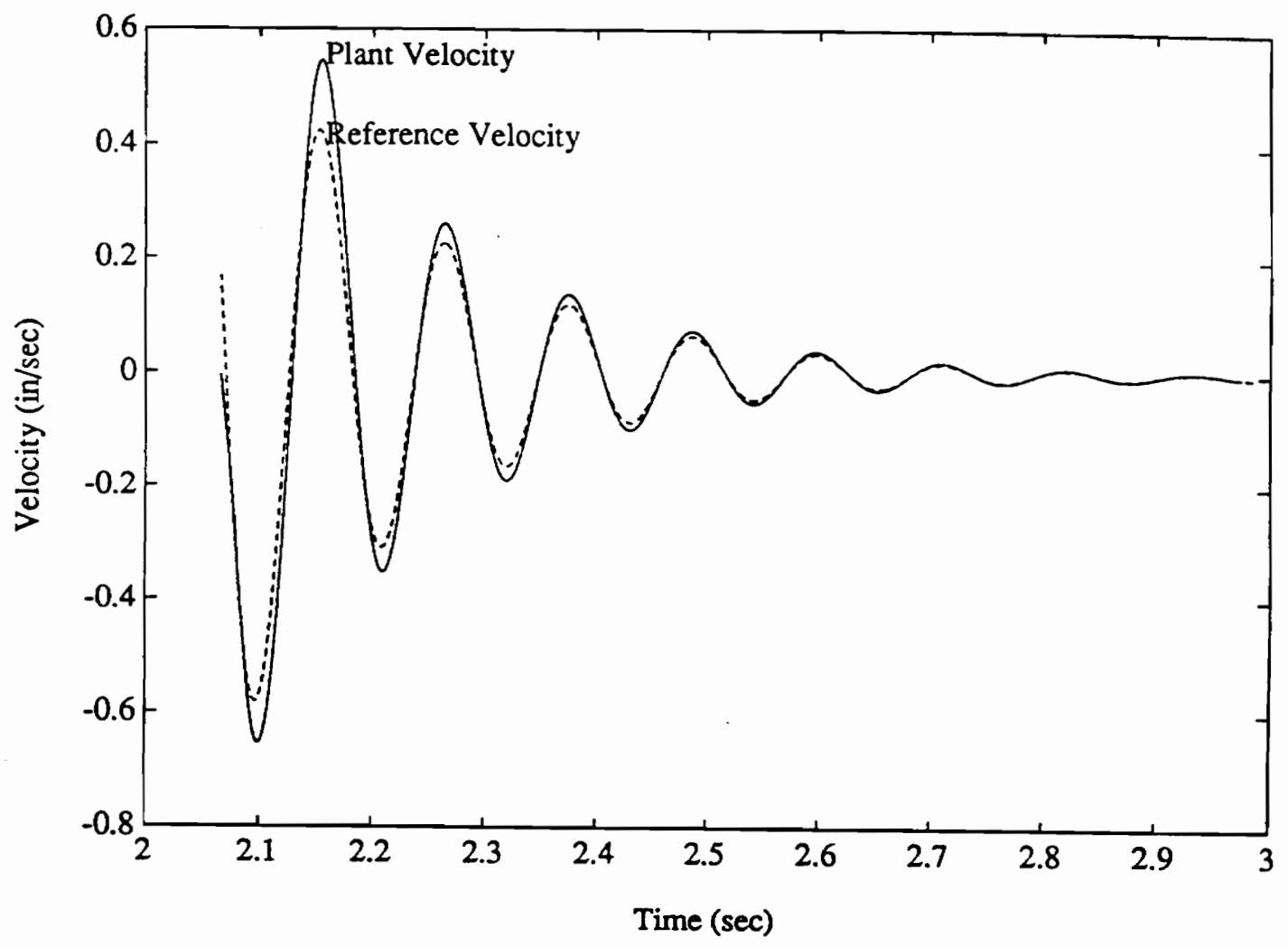

Figure 5-4

Shifted $\left(t_{d}=0.01 \mathrm{sec}\right)$ Analytical Velocity Response of the Suspended Mass System $\left(\alpha=1.5, T=50.0[I]\right.$, and $\left.T^{*}=[I]\right)$ 


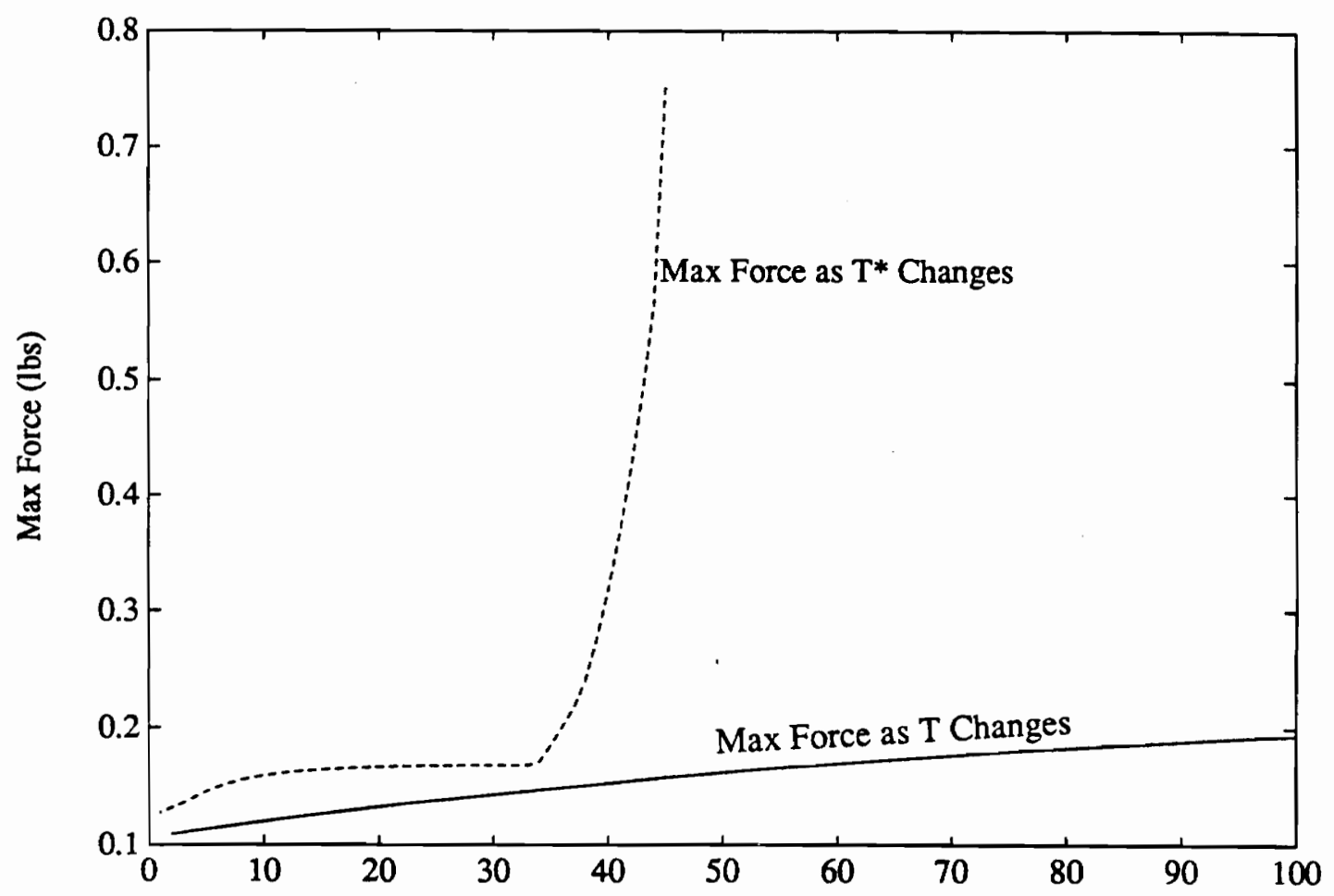

Multiplier $\lambda$

Figure 5-5 Analytical Maximum Force for Different Diagonal $\mathbf{T}$ and $\mathbf{T}^{*}$ $T=\lambda\left[\Pi, T^{*}=[I]\right.$ or $T=16 I, T^{*}=\lambda I$ (Suspended Mass System, $\alpha=1$ ) 


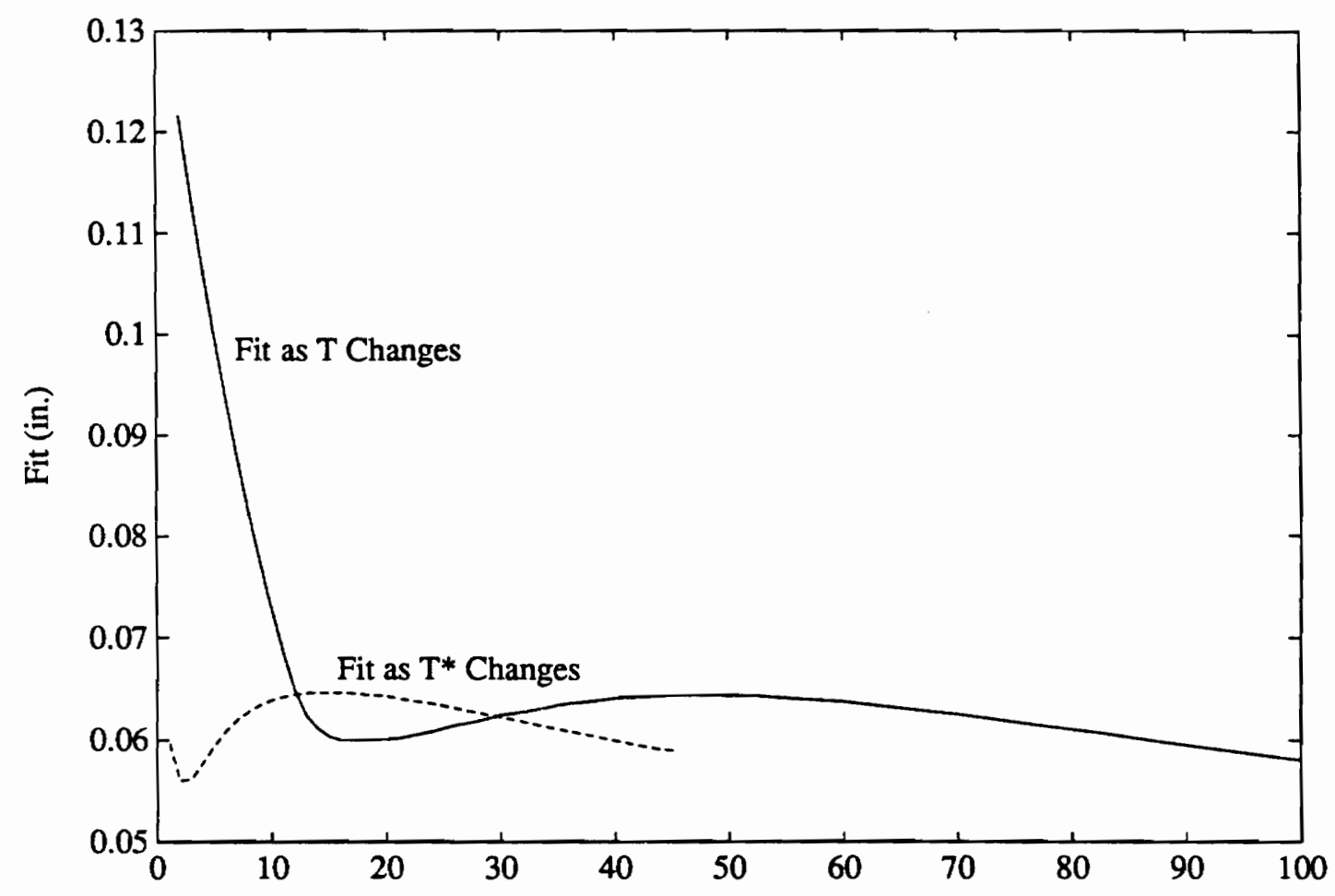

Multiplier $\lambda$

Figure 5-6

Analytical Fit for Different Diagonal $T$ and $\mathbf{T}^{*}$

$T=\lambda[I], T^{*}=[I]$ or $T=16 I, T^{*}=\lambda I$ (Suspended Mass System, $\alpha=1$ ) 


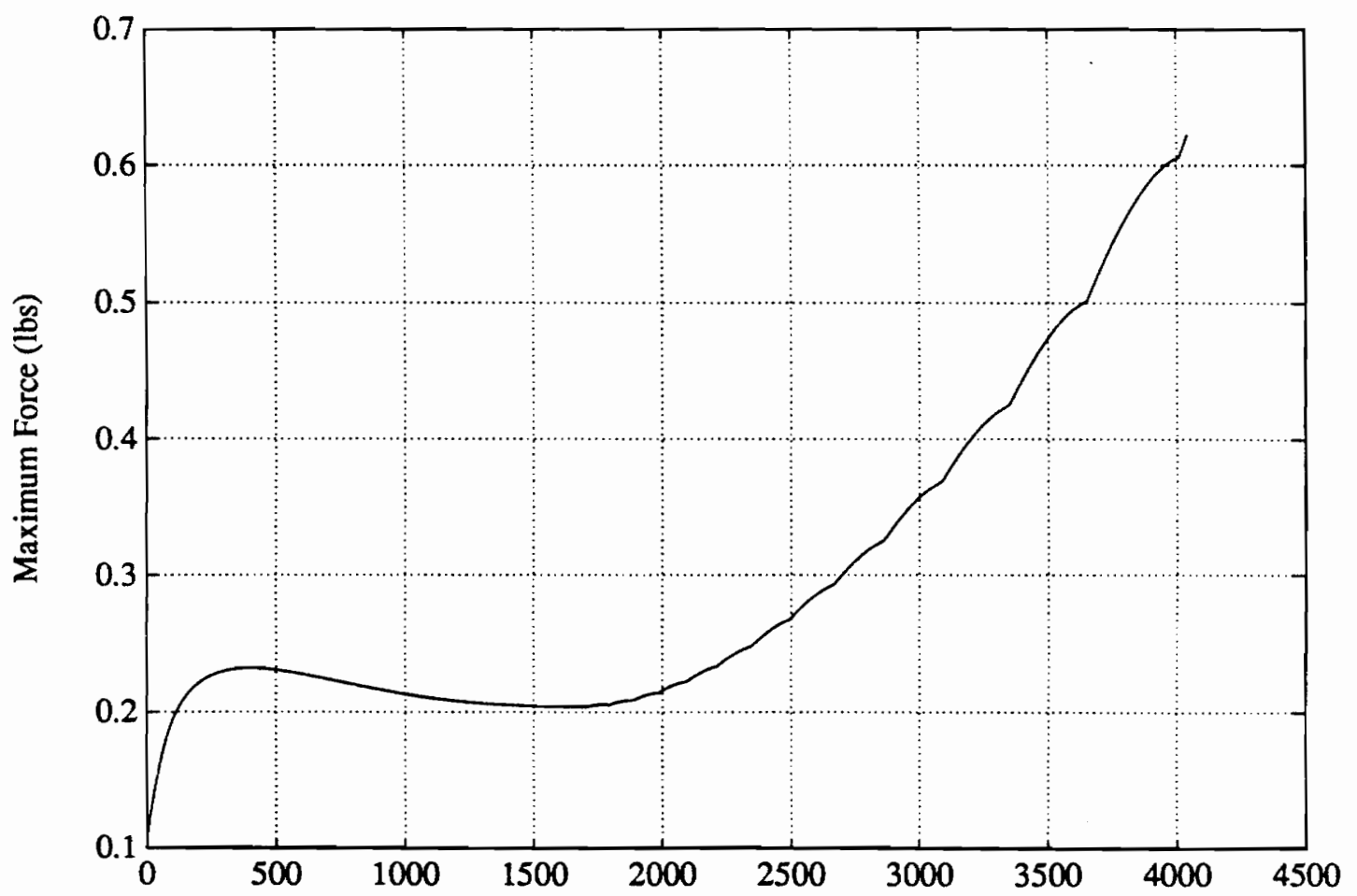

Multiplier $\lambda$

Figure 5-7

Analytical Maximum Force for Different Diagonal $T$ and $\mathbf{T}^{*}$ $T=\lambda[I], T^{*}=[I]$ (Suspended Mass System, $\alpha=1$ ) 


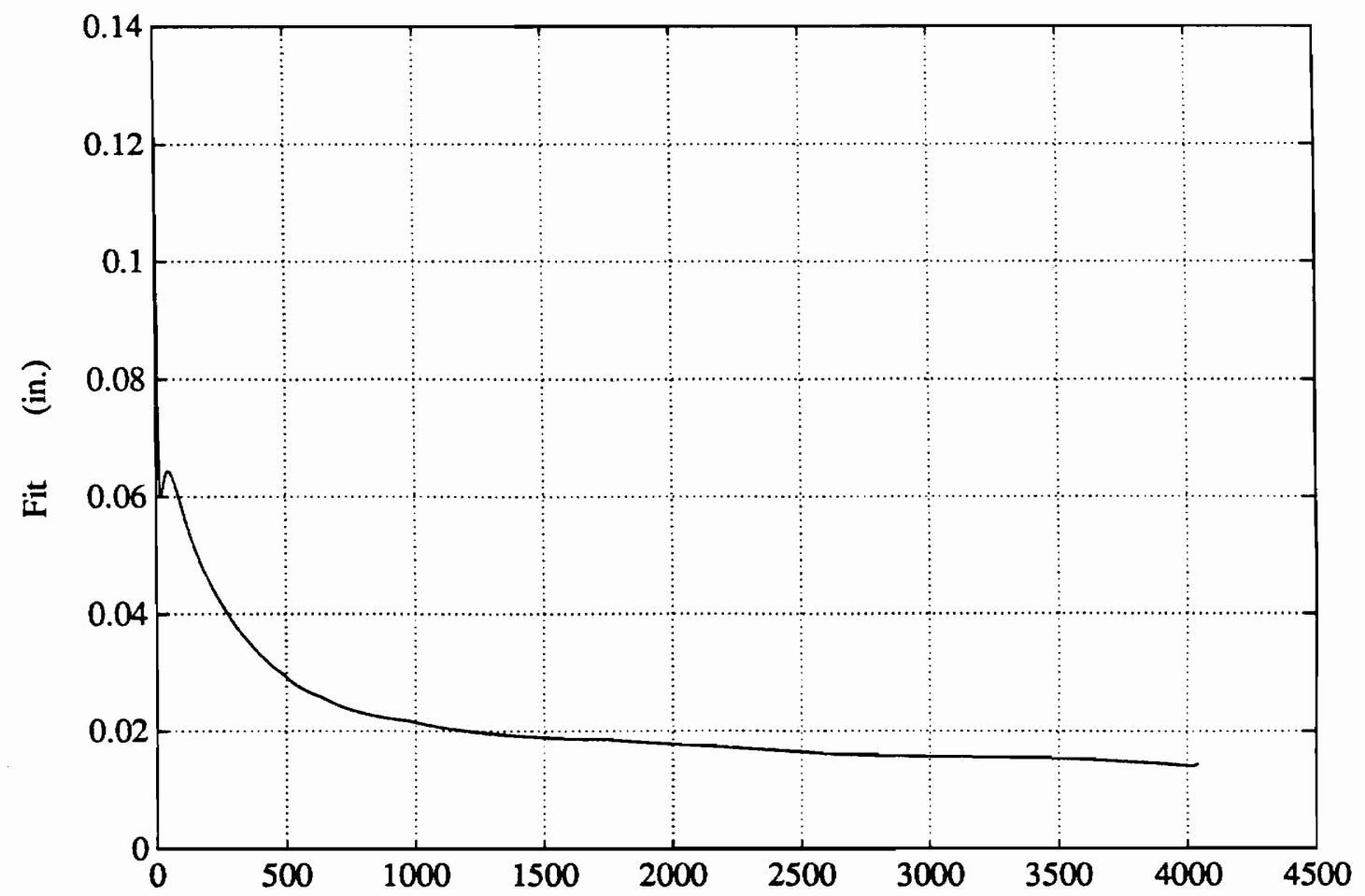

Multiplier $\lambda$

Figure 5-8

Analytical Fit for Different Diagonal $\mathbf{T}$ and $\mathbf{T}^{*}$

$\mathbf{T}=\lambda[\mathbf{I}], \mathbf{T}^{*}=[\mathrm{I}]$ (Suspended Mass System, $\left.\alpha=1\right)$ 


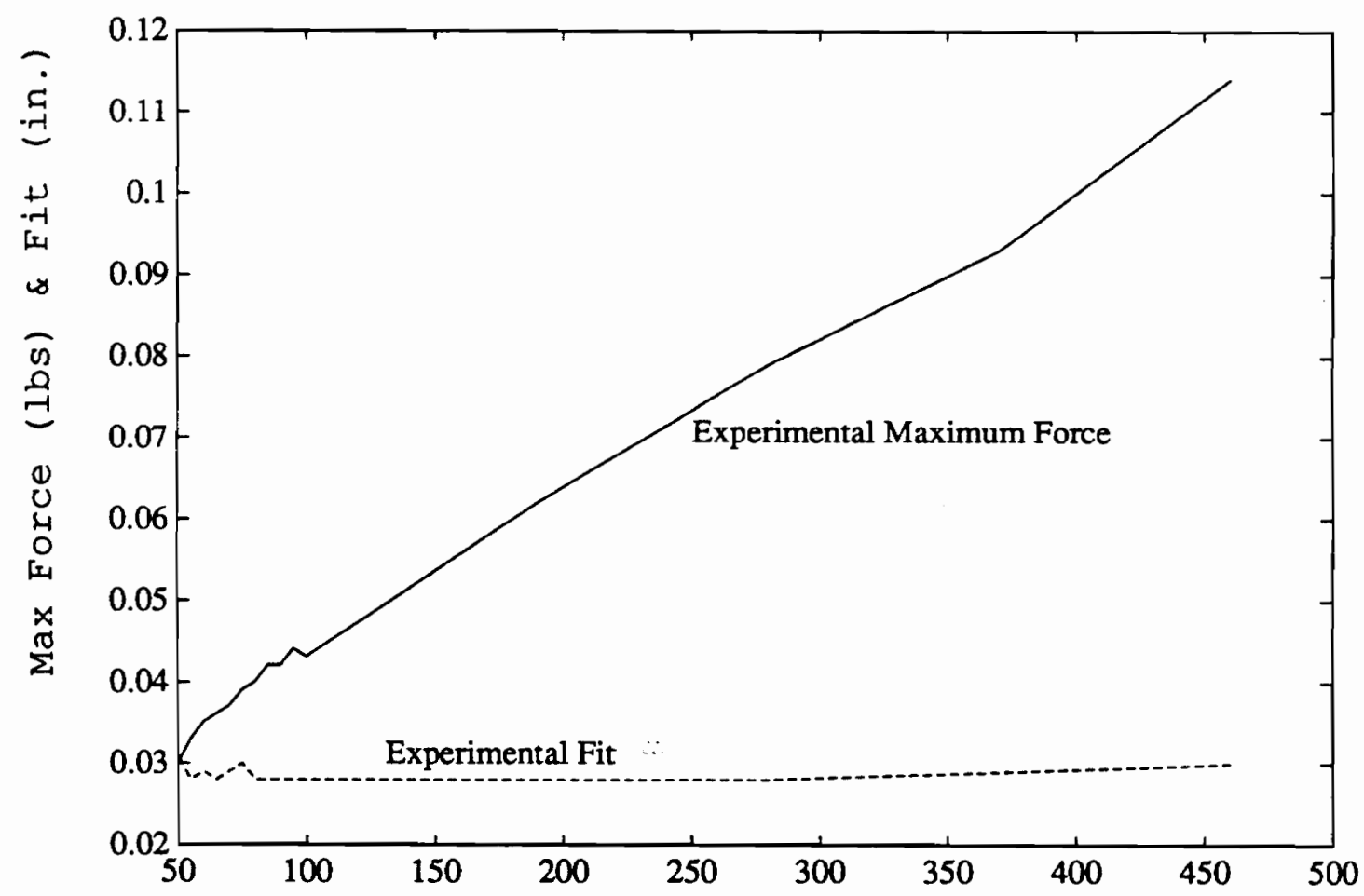

Multiplier $\lambda$

Figure 5-9

Experimental Maximum Force for Different Diagonal $T$ and $\mathbf{T}^{*}$ $\mathbf{T}=\lambda[\mathbf{I}], \mathbf{T}^{*}=[\mathrm{I}]$ (Suspended Mass System, $\alpha=1$ ) 


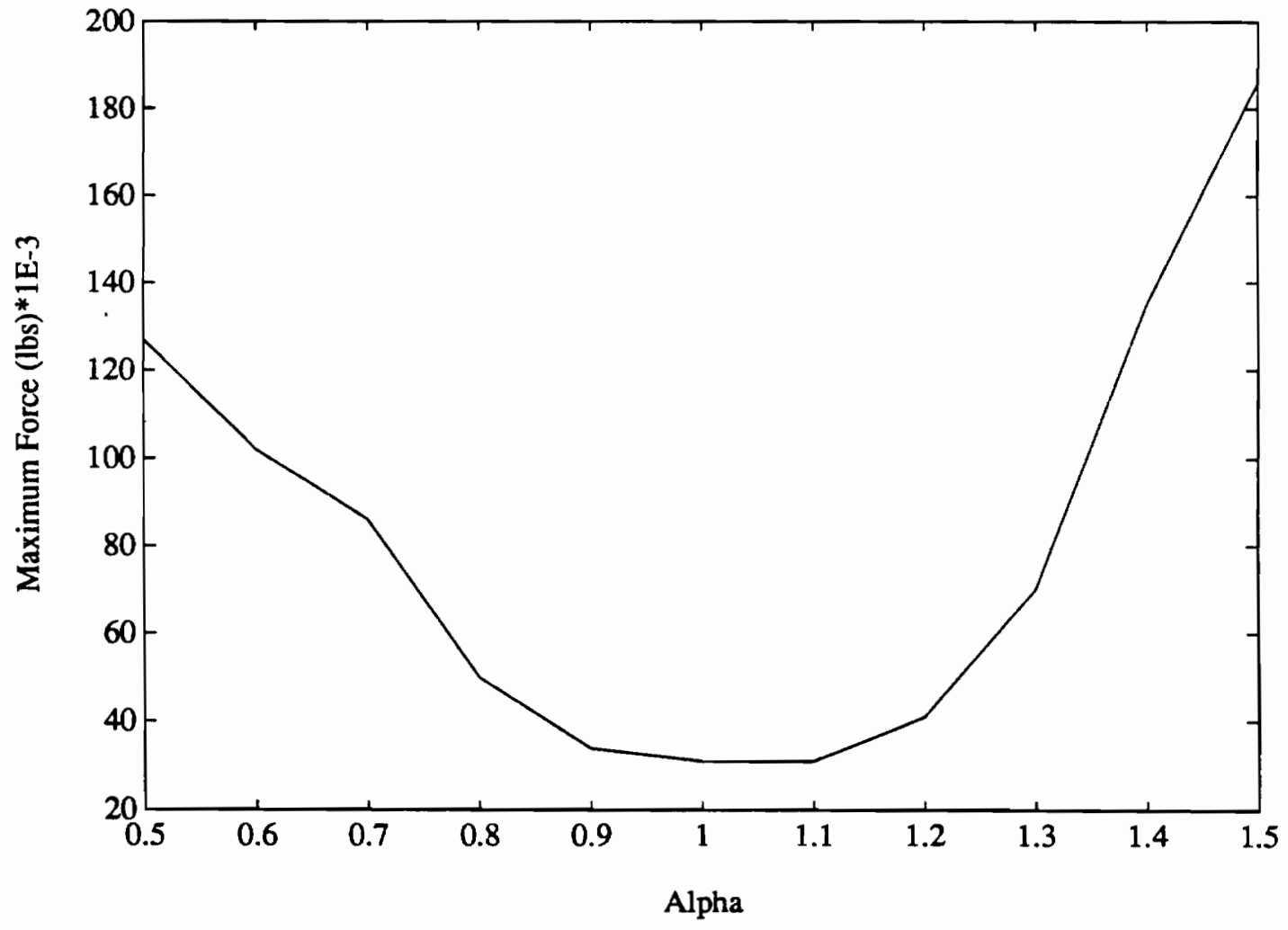

Figure 5-10

Experimental Maximum Force For Different Values of Alpha $\mathbf{T}=50[\mathrm{I}], \mathbf{T}^{*}=[\mathrm{I}]$ (Suspended Mass System) 


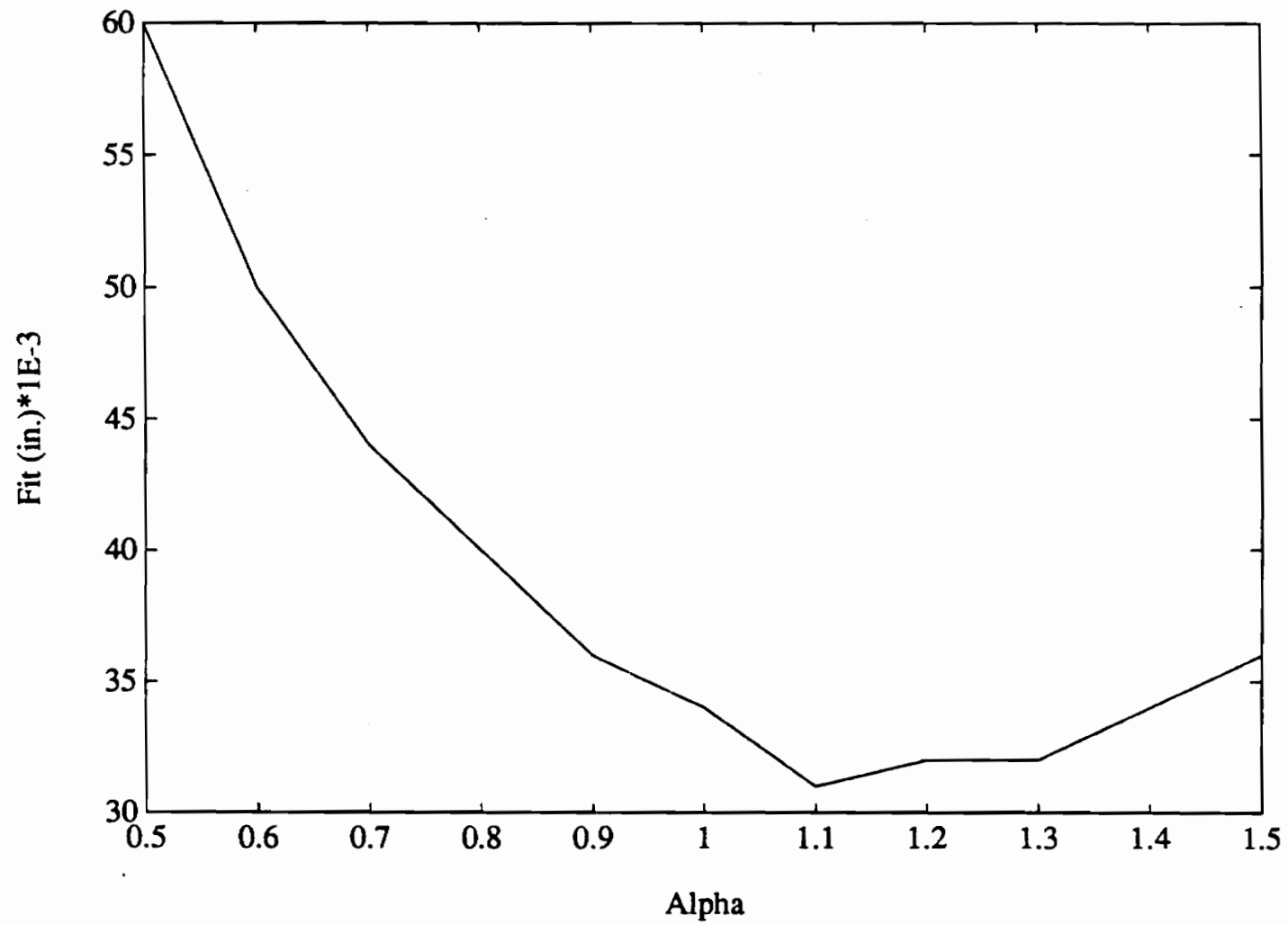

Figure 5-11

Experimental Fit for Different Values of Alpha

$\mathbf{T}=50[\mathrm{I}], \mathbf{T}^{*}=[\mathrm{I}] \quad$ (Suspended Mass System) 


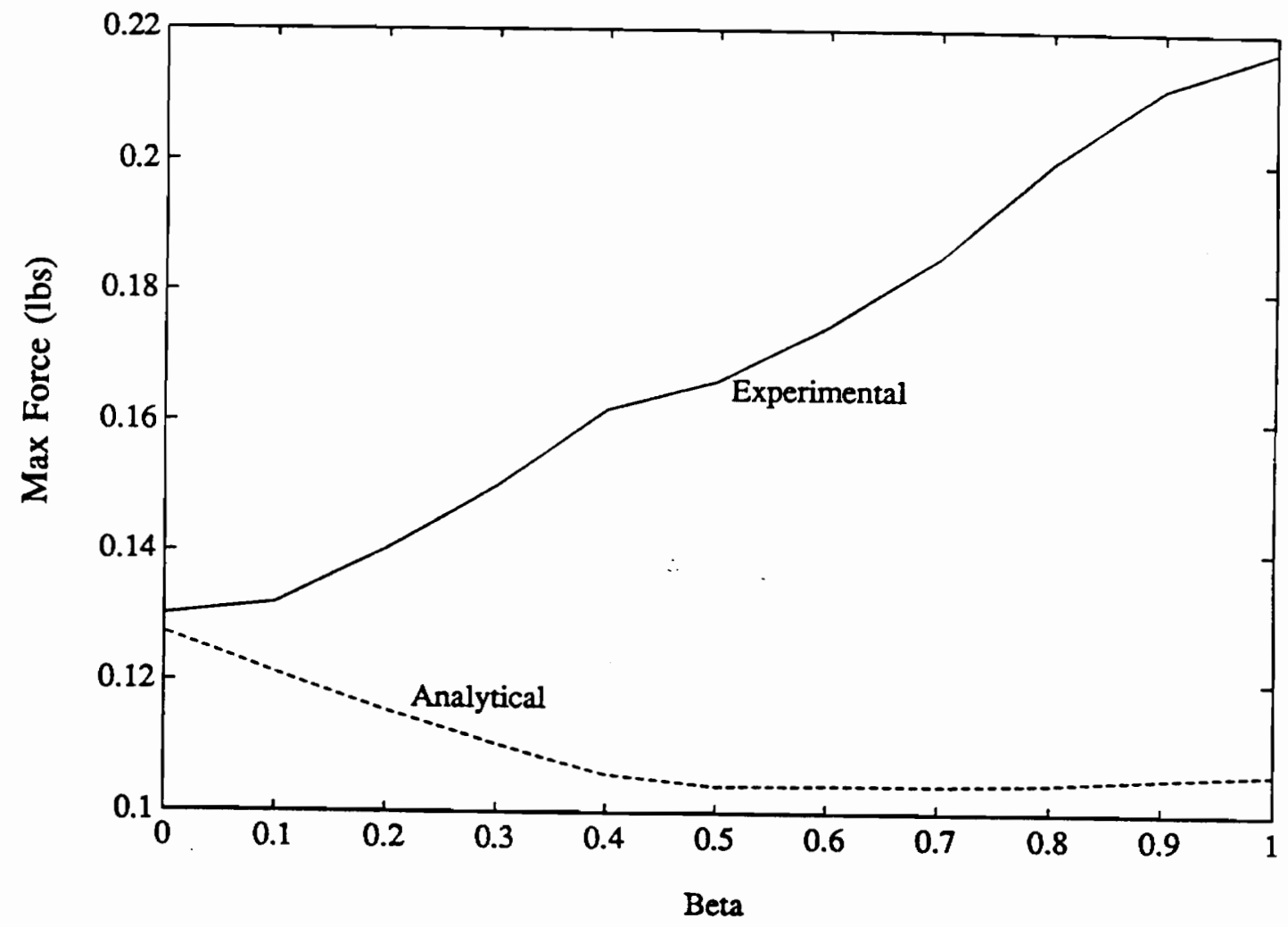

Figure 5-12

Experimental and Analytical Maximum Force for Intermediate Design Points

Between Case 2 Optimal Design, and Case 3 Optimal Design

(Suspended Mass System) 


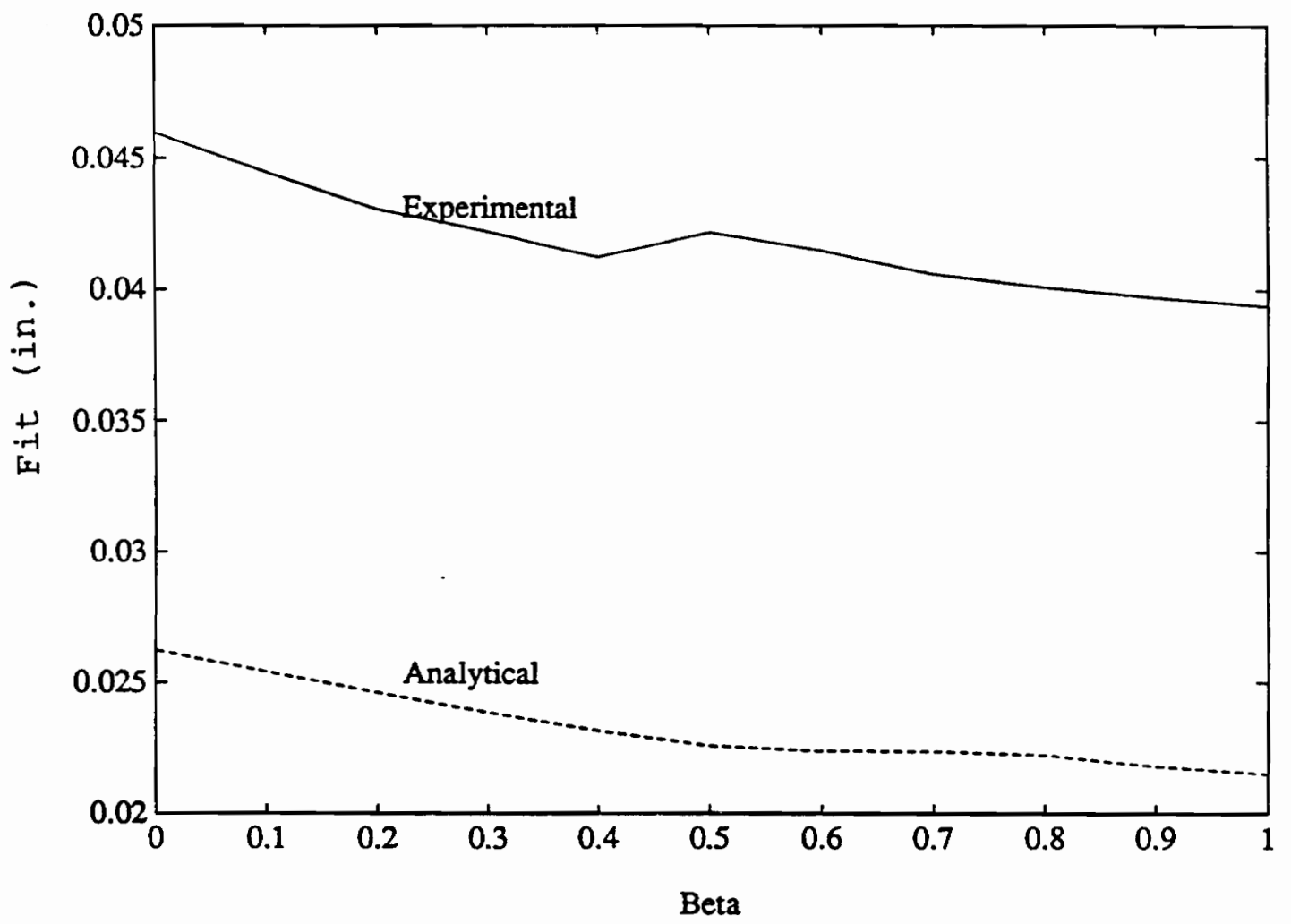

Figure 5-13

Experimental and Analytical Fit for Intermediate Design Points

Between Case 2 Optimal Design, and Case 3 Optimal Design

(Suspended Mass System) 


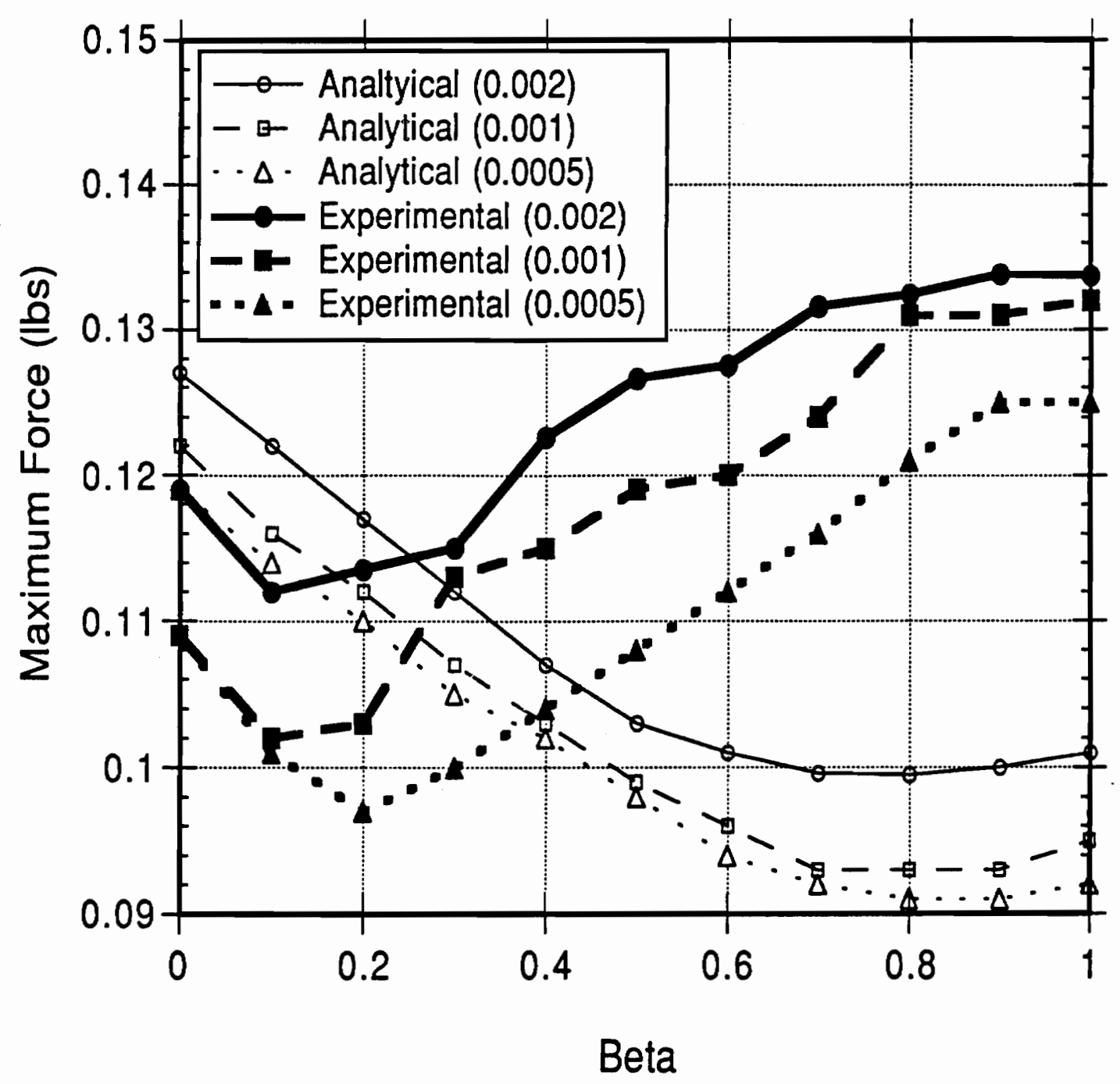

Figure 5-14

Experimental \& Analytical Maximum Force for Varying Sizes of the Off Diagonal Elements $T^{*}(1,2) \& T^{*}(2,1)$, and Sampling Rates

(Suspended Mass System, $\alpha=1.5$ ) 


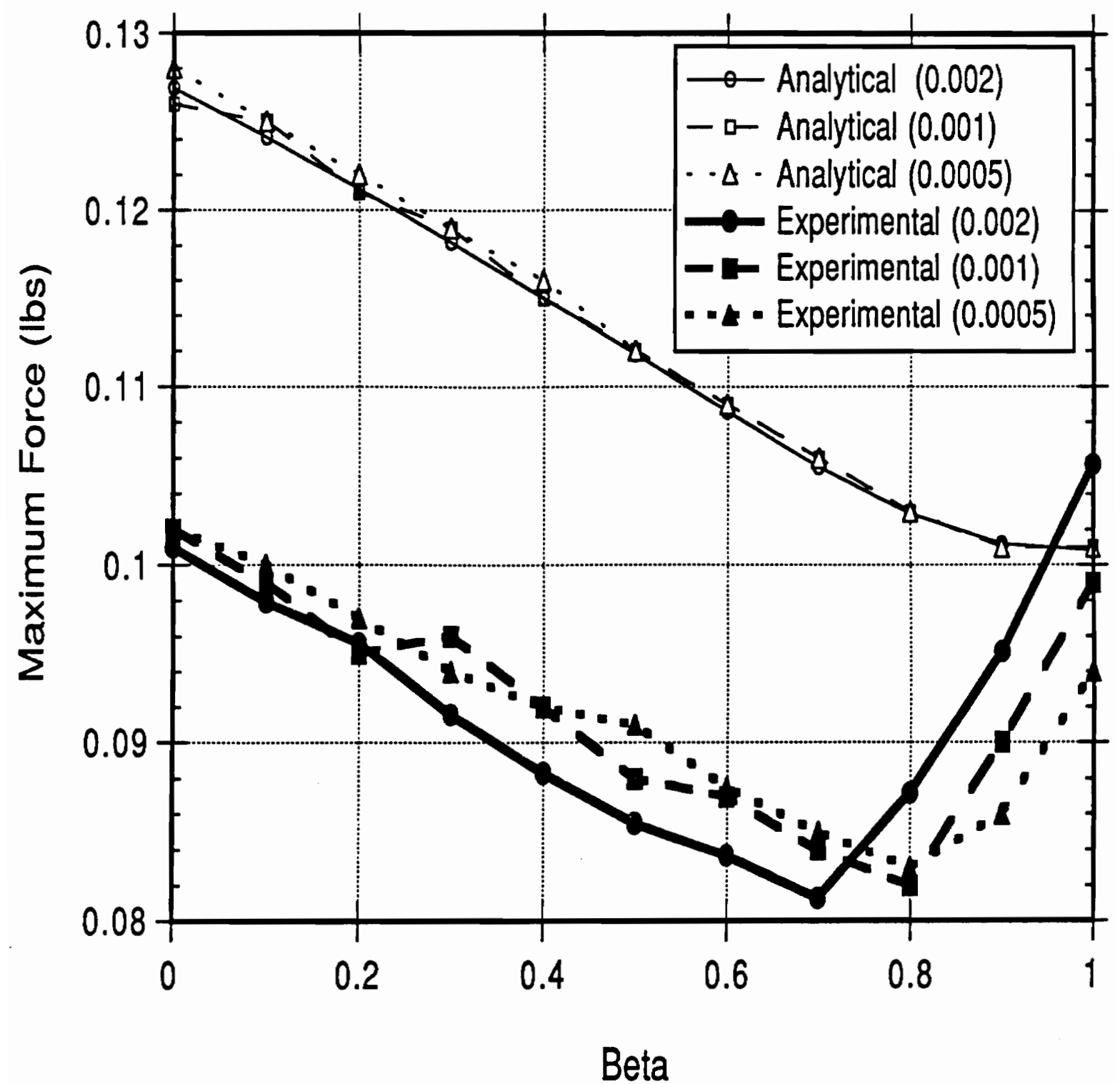

Figure 5-15

Experimental \& Analytical Maximum Force for Varying Sizes of the Off Diagonal Elements $T^{*}(1,2) \& T^{*}(2,1)$, and Sampling Rates

(Suspended Mass System, $\alpha=0.5$ ) 


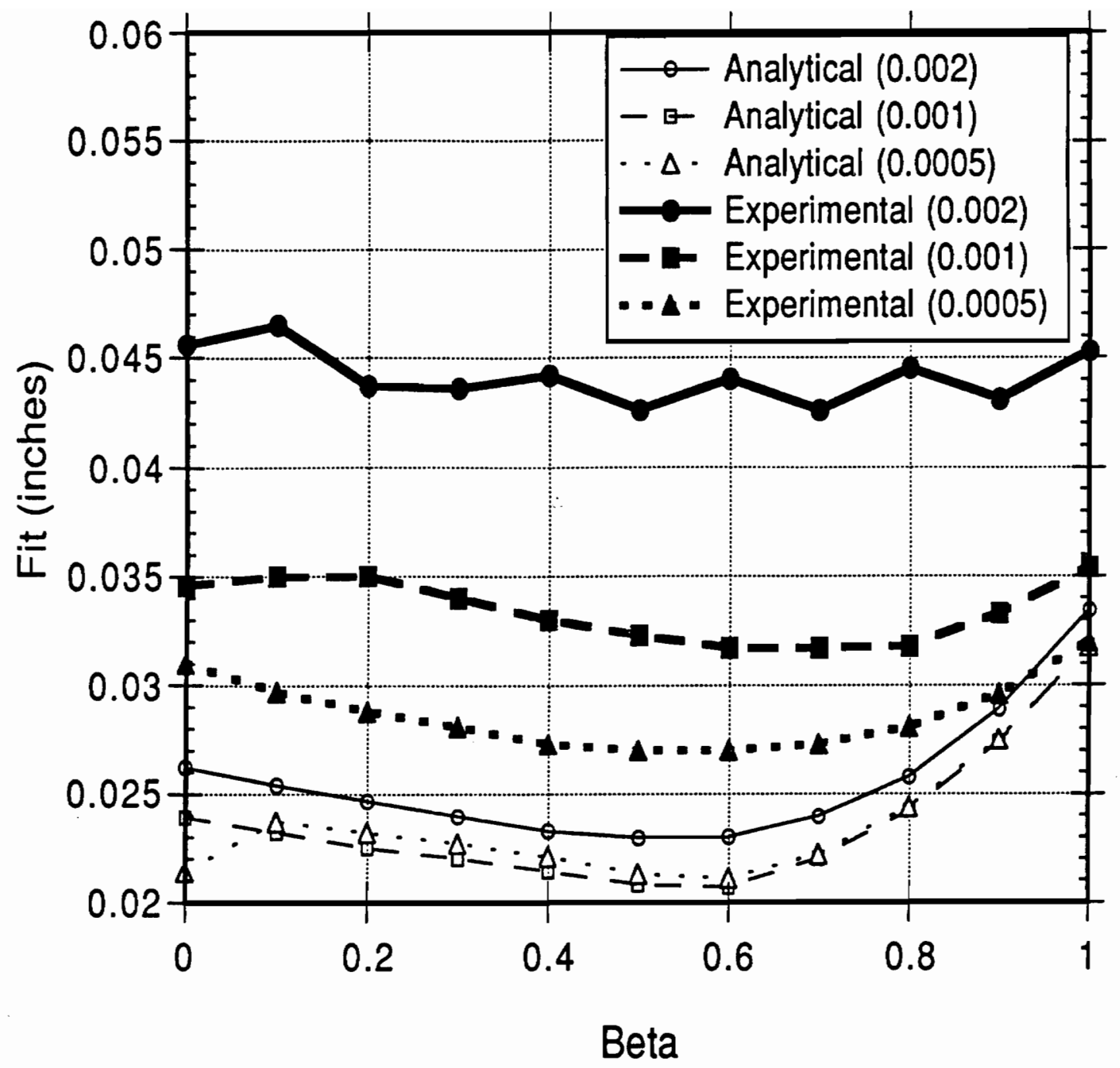

Figure 5-16

Experimental \& Analytical Fit for Varying Sizes of the Off Diagonal Elements $T^{*}(1,2) \& T^{*}(2,1)$, and Sampling Rates

(Suspended Mass System, $\alpha=1.5$ ) 


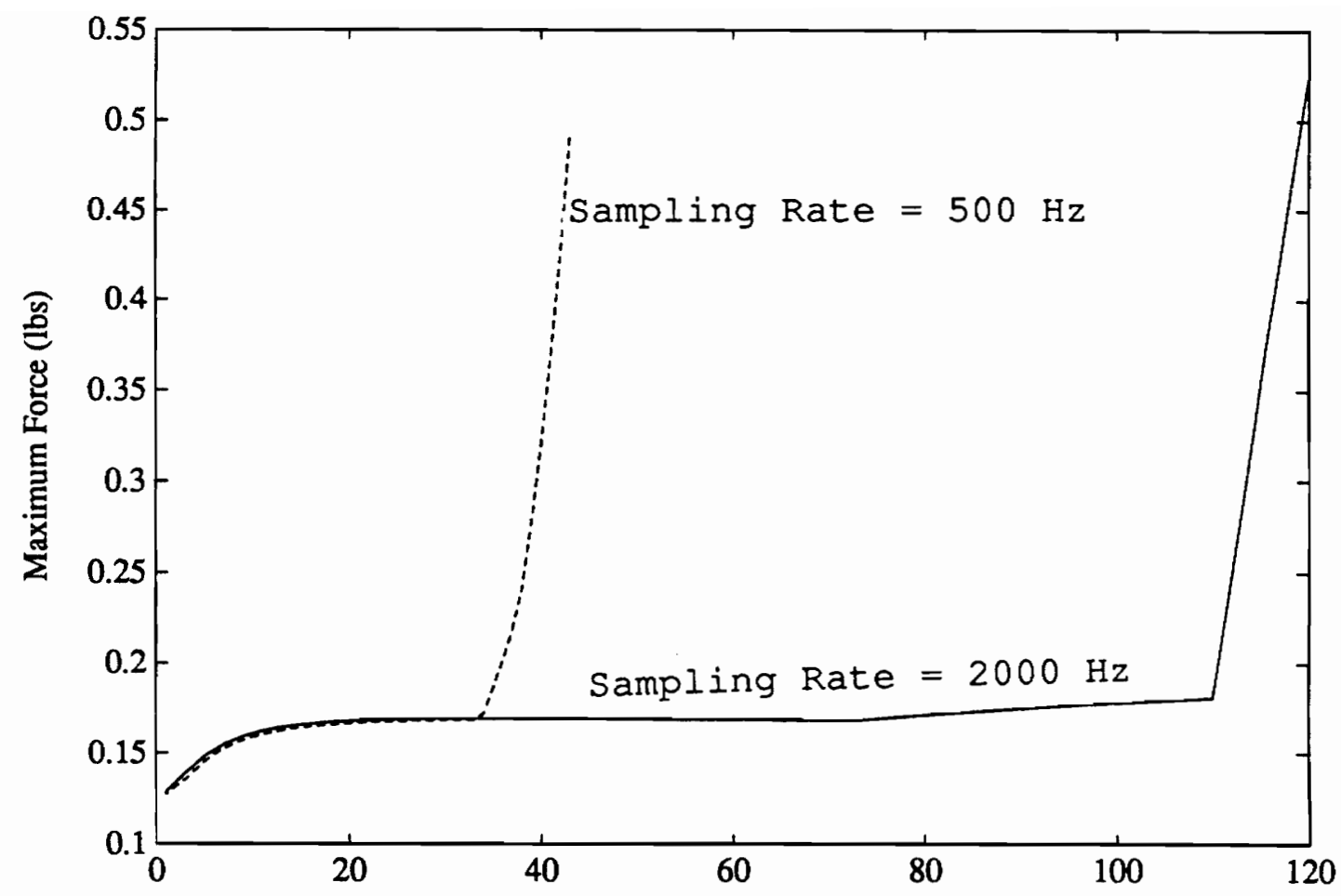

Multiplier $\lambda$

Figure 5-17

Analytical Maximum Force for Different Diagonal $\mathbf{T}^{*}$

$T=16[I], T^{*}=\lambda[I]$ (Suspended Mass System $\alpha=1$ ) 


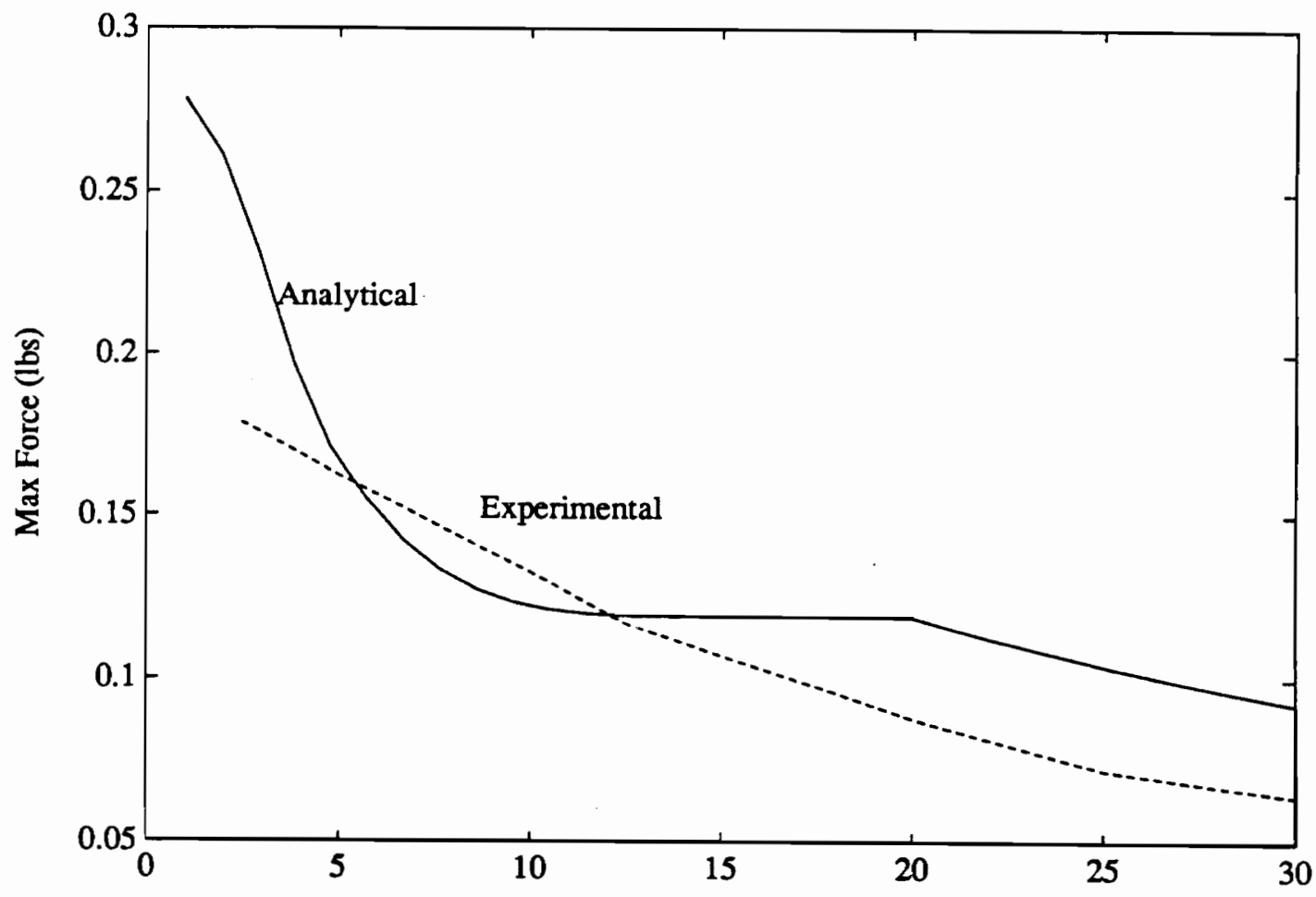

Percent Damping in Reference Model

Figure 5-18

Experimental and Analytical Effects of Damping on the Maximum Force (Suspended Mass, $\alpha=0.5, T \& T^{*}$ From Optimal Design Case 2) 


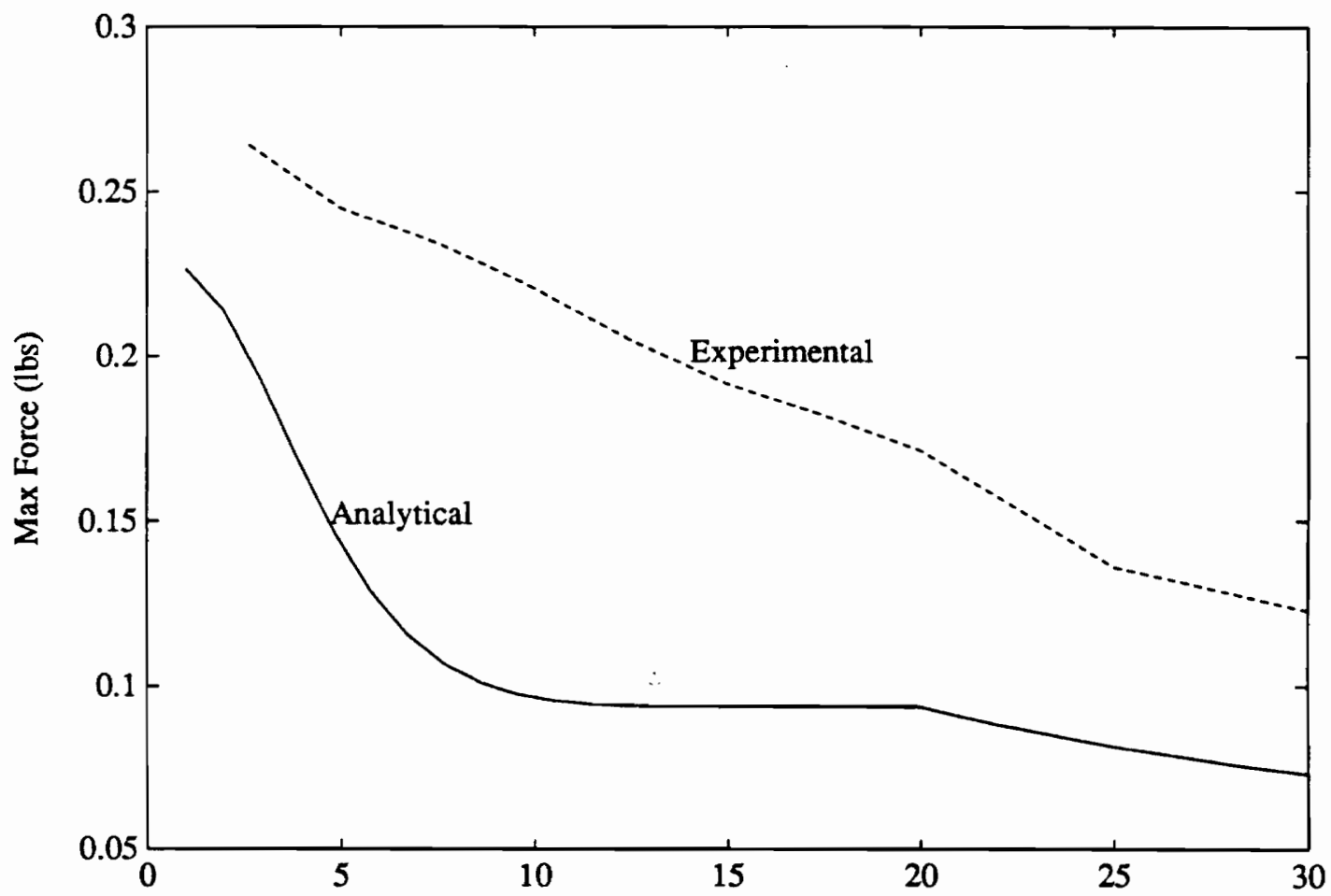

Percent Damping in Reference Model

Figure 5-19

Experimental and Analytical Effects of Damping on the Maximum Force (Suspended Mass, $\alpha=1.5, T \& T^{*}$ From Optimal Design Case 2) 


\section{Chapter 6 \\ Concluding Remarks}

The performance of MRAC was studied in numerical simulations and verified experimentally to understand how differences between the plant and the reference model affect the control effort. MRAC was applied analytically and experimentally to a single degree of freedom system and analytically to a multi degree of freedom system with multi-inputs and multi-outputs. Good experimental and analytical agreement was demonstrated in control experiments and it was shown that MRAC does an excellent job of controlling the structures and achieving the desired performance even when large differences between the plant and reference model exist. However, reasonable errors between the reference model and the plant can significantly increase the required control effort as well as the maximum required force.

The effects of increased damping in the reference model were considered, and it was shown that requiring the controller to provide more damping actually decreased the required maximum force when differences between the plant and reference model exist. This result is very useful because one of the first attempts to counteract the increased maximum force due to difference between the plant and reference model might be to require less damping, which would actually compound the problem.

Finally, the use of optimization to improve performance and reduce cost is limited. It was shown that optimization of the diagonal elements of the weighting matrices and the required damping did yield both experimental and analytical improvement in the design. However, when off diagonal elements were included, the correlation between experimental and analytical results was poor. The problem appears to be associated with the sampling rate. It may indicate that sampling rate for MRAC may need to be very high to achieve good analytical and experimental agreement. 


\section{Appendix A \\ Derivation of Integration Algorithm}

Given

$$
\dot{X}=f(X),
$$

we can expand with a Taylor Series about $\mathrm{X}^{\mathrm{n}+1}$

$$
X^{n}=X^{n+1}-\Delta t \dot{X}^{n+1}+\frac{\Delta t^{2}}{2} \dot{X}^{n+1}+\theta(\Delta t)^{3} .
$$

Now, since

$$
\begin{aligned}
& \dot{X}=f(X), \\
& \dot{X}=f(x),
\end{aligned}
$$

we can write

$$
X^{n}=X^{n+1}-\Delta t f^{n+1}+\frac{\Delta t^{2}}{2} f^{n+1}+\theta(\Delta t)^{3} .
$$

If we now substitute

$$
E=\frac{f^{n+1}-f^{n}}{\Delta t}
$$

eq. A-4 can be simplified to

$$
X^{n+1}-X^{n}-\frac{\Delta t}{2}\left[f^{n+1}+f^{n}\right]=0 .
$$

Noting that eq A-6 has the form $r(x)=0$, we can apply Newton's iteration Derivation of the Integration Algorithm 


$$
I^{n+1}+\left(\frac{\partial r^{n+1}}{\partial X^{n+1}}\right) \Delta x=0
$$

where $\Delta \mathrm{X}$ is an estimate of the change in $\mathrm{X}$. When the magnitude of all terms in $\Delta \mathrm{X}$ becomes small, the iteration process has converged. Taking the necessary derivatives and collecting terms we can write

$$
\left[\frac{\Delta t}{2} \frac{\partial f^{n+1}}{\partial X^{n+1}}-I\right] \Delta X=X^{n+1}-X^{n}-\frac{\Delta t}{2}\left[f^{n+1}+f^{n}\right]
$$

where $\partial \mathrm{f}^{\mathrm{n}+1} / \partial \mathrm{X}^{\mathrm{n}+1}$ is the Jacobian matrix. Although it would be possible for the simulation algorithm to update the plant velocities, and thus the elements of $e_{y}$, at each iteration, this would not be consistent with the digital effects of the DSP board. In order to simulate the exchange of information between the DSP and the plant, the elements of $e_{y}$ and the plant velocities used in the Jacobian matrix are held constant through each iterative step. Solving for $\Delta \mathrm{X}$ the final update equation is written

$$
\Delta X=\left[\frac{\Delta t}{2} \frac{\partial f^{n+1}}{\partial x^{n+1}}-I\right]^{-1}\left[x^{n+1}-x^{n}-\frac{\Delta t}{2}\left(f^{n+1}+f^{n}\right)\right]
$$

It is noted, that $\Delta \mathrm{t}$ is the sampling rate of the control system, and not just related to accuracy considerations 


\section{Appendix B}

\section{Slewing Grid: Description and Finite Element Analysis}

An extensive research effort went into correlating the analysis of the Slewing Grid structure with the results obtained by experimental modal analysis. However, the correlation between analysis and experiments for this structure is not good. This appendix describes the finite element analysis procedures used, and some of the attempts to improve the analysis. The appendix begins with a complete description of the physical components of the Slewing Grid, and is followed by a discussion of the static analysis performed. Next, the finite element model used to describe the dynamics of the structure is discussed, and finally the experimental/analytical results are compared.

\section{B.1 Slewing Grid Description}

The Virginia Tech Slewing Grid laboratory structure is a more complex structure designed to have several two-dimensional out-of-plane low frequency modes of vibration, The structure was designed to have a zero frequency rigid body rotation about the rotation shaft, but imperfections and friction prevented its realization. The grid is composed of five lightweight aluminum members $2 \times 1 / 8$ inch cross sections and bolted together at each of the five joints. The members range in length from 60 to 86 inches with the longest unsupported length being 61.5 inches for the top and bottom horizontal members. The rotation shaft is a 1.3 inch circular steel shaft, 90.0 inches long and supported by two bearings 76.75 inches apart. The bearings are in turn, held in place by two brackets which are bolted to a large piece of channel-section connected to the laboratory wall. Three pairs of velocity sensors and force actuators are colocated at joints 3,4 and 5 . 


\section{B.2 Static Analysis}

Although the geometry of the structure is symmetric about a horizontal line through joint 3 , the vibration mode shapes are not similarly symmetric because the structure's weight causes asymmetric member gravity loading and therefore an asymmetric stiffness distribution. Due to gravity loading, the lower horizontal member is compressed to about $70 \%$ of its Euler (pin-ended) buckling load. Great effort was taken to accurately predict the loads in each member of the structure. However, after the structure was assembled, a nut and bolt assembly at each joint was tightened. The process of tightening the nut and bolt assembly induced unknown forces into the members. Strain gages were mounted on several members in an attempt to measure these forces. The configuration of the strain gages (fig. B-1) was designed so the axial force as well as the moments about the $\mathrm{y}$ and $\mathrm{z}$ axis could be calculated separately as

$$
\begin{gathered}
M_{y}=\left[\frac{\epsilon_{3}-\epsilon_{1}}{2}\right] \frac{E I_{y y}}{Z}, \\
M_{z}=\left[\frac{\epsilon_{2}-\epsilon_{1}}{2}\right] \frac{E I_{z z}}{Y}, \\
T=\left[\frac{\epsilon_{2}+\epsilon_{1}}{2}\right] E A .
\end{gathered}
$$

where $M_{z}$ and $M_{y}$ are the moments about the strong and weak axis respectively, $T$ is the axial force, $\varepsilon_{i}$ is the strain measured by the $i^{\text {th }}$ gage, $I_{y y}$, and $I_{z z}$ are moments of inertia about the respective axis, $\mathrm{E}$ is the modules of elasticity, $\mathrm{A}$ is the cross sectional area and $\mathrm{Y}$, and $\mathrm{Z}$ are distances away from the respective axes. However, because the strain in the weak direction was so large compared to strain in the axial direction, calculation of the increase in axial stress was abandoned. It was also discovered that 
the process of tightening the nut and bolt assembly was not repeatable (even though the same torque was applied each time). Therefore, the only conclusion reached from this study, was that the value of the moments induced by tightening the bolts was on the same order of magnitude as those produced by gravity loading.

\section{B.3 Finite Element Model (FEM)}

The Slewing Grid structure was modeled by 24 finite elements connected at 27 grid points labeled on fig. B-2. The aluminum member are each divided into 4 grid elements with 3 Degrees Of Freedom (DOF) at each joint. The DOF are (See fig. B3) out of plane translation, and out of plane rotations about both in-plane axes. As seen from fig. B-3, the finite element also takes into account a rigid section on the ends so as to model the area near the nut and bolt assembly. The rotational shaft is divided into 8 beam elements with 2 DOF at each joint, out-of-plane displacement and out-of-plane rotation. The finite elements model has a total of 72 DOF.

The element mass and stiffness matrices used for the aluminum grid are standard, with bending deformation based on Euler-Bernoulli theory with cubic shape functions, and torsional deformations based on linear shape functions (ref. 51). A geometric stiffness matrix is used to account for axial forces, shear forces and bending moments on each member. It should be noted, however, that the geometric stiffness matrix assumes the forces and moments to be constant along the length of the element. Although this is not true for the Slewing Grid, the changes in these forces across a given element are small, so an average value of the force and moments was used. Because the forces due to bolt tightening at the joints are essentially unknown, only the gravity induced forces are included in the geometric stiffness matrix. A torsional spring element was added to the global stiffness matrix to account for the friction in the bearings. The value of this torsional spring was determined by forcing the lowest analytical frequency to match the lowest measured frequency. The mass matrix is a consistent mass matrix with point masses included to account for the structure-borne 
sensor and actuator coils. The damping matrix of the Slewing grid is unknown but the inherent damping is very small and is therefore not included. The equation of motion for the FEM are written as

$$
M \bar{X}(t)+(K+G) X(t)=F(t)
$$

where $M$ and $K$ are the mass and beam stiffness matrix, $G$ is the geometric stiffness matrix and $\mathrm{F}$ is the forcing matrix.

\section{B.4 Experimental/Analytical Modal Analysis Results}

The accuracy of the frequencies and mode shapes predicted by the FEM is not good. Over a two year period, much effort was devoted to developing and refining a FEM model which would accurately model the Slewing Grid structure. However, the non-linearities in the structure, such as friction in the bearings, large gravity loading in the lower horizontal member, and the unknown loads induced by tightening the bolts at each joint, have been difficult to model. Table 3-1 compares the first ten frequencies predicted by the most refined FEM to the experimental vibration frequencies, and figs. 3-6 to 3-12 compare several analytical and experimental mode shapes. Table 3-1 and figure 3-9 show that the major discrepancy between experiments and analysis is for the fourth mode. Efforts to explain this difference first centered on non-linearities because of the large compressive load in the lower horizontal member. As mentioned previously, gravity loading alone caused the lower horizontal member to be stressed to $70 \%$ of it Euler (pin-ended) buckling load. It is well known that a compressive load in a structural member should lower its vibration frequency but the measured frequency was $20 \%$ higher instead of lower. One possible explanation is that the member behaves like a shallow arch instead of a beam. This hypothesis is supported by three observations. First, because of the large compressive load, the lower horizontal member has a 0.125 inch initial displacement at the center when the structure is in static equilibrium. Second, during dynamic testing the lower 
horizontal member exhibited sporadic snap through. Finally, the measured shape of the lower horizontal member and the higher measured frequency are both consistent with the fundamental mode of a shallow arch.

Using standard finite element methods to model the dynamics of the Slewing Grid has produced analytical frequencies and mode shapes which do not agree well with experimentally measured frequencies and mode shapes. One possible solution is to use model correction techniques to further improve the mathematical model. One such model correction method developed by Baruch (ref. 17) is discussed in appendix C 


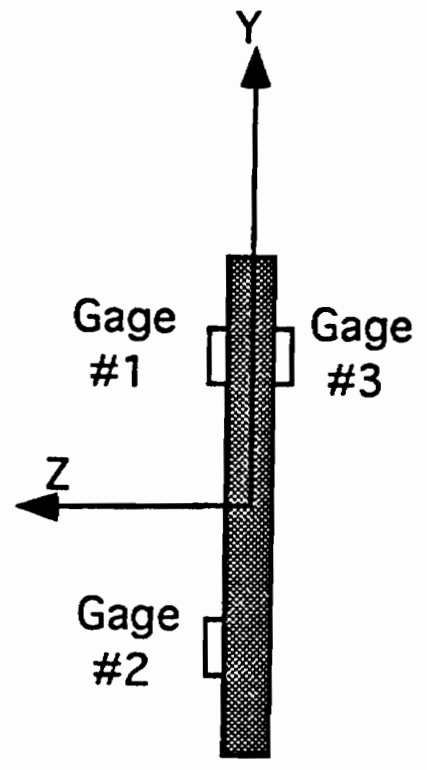

End View

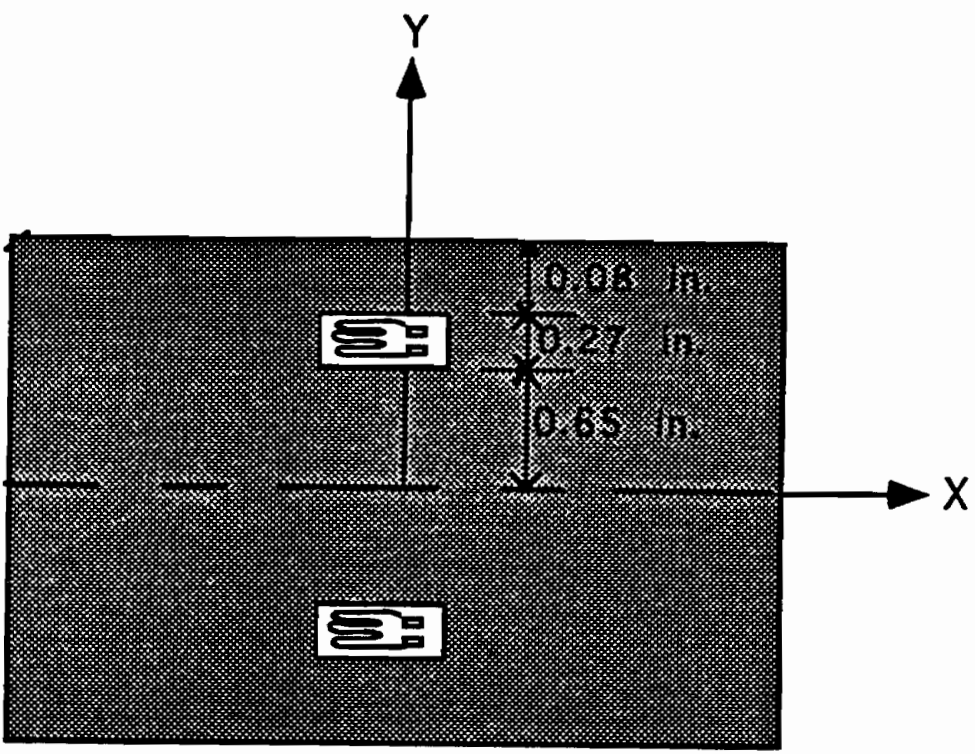

Side View

Strain Gage Information:

Manufacture: Micro-Measurements

Gage Type: CEA-13-125UW-350

Figure B-1

Configuration of The Strain Gages Mounted on Members of The Slewing Grid to Calculate Forces Due to Tightening of Bolts 


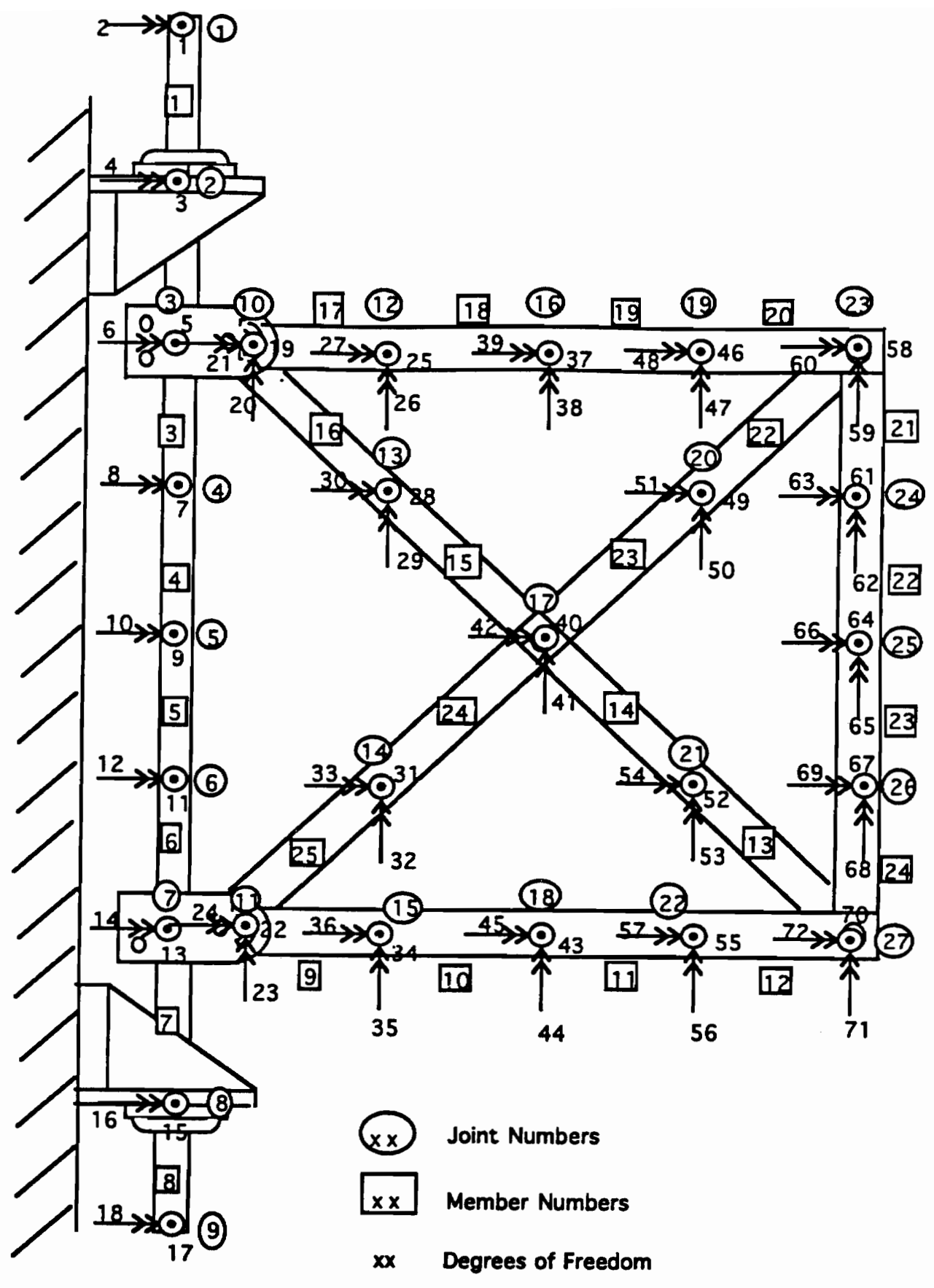

Figure B-2

Numbering Scheme For The Finite Element Model of the Slewing Grid Structure 


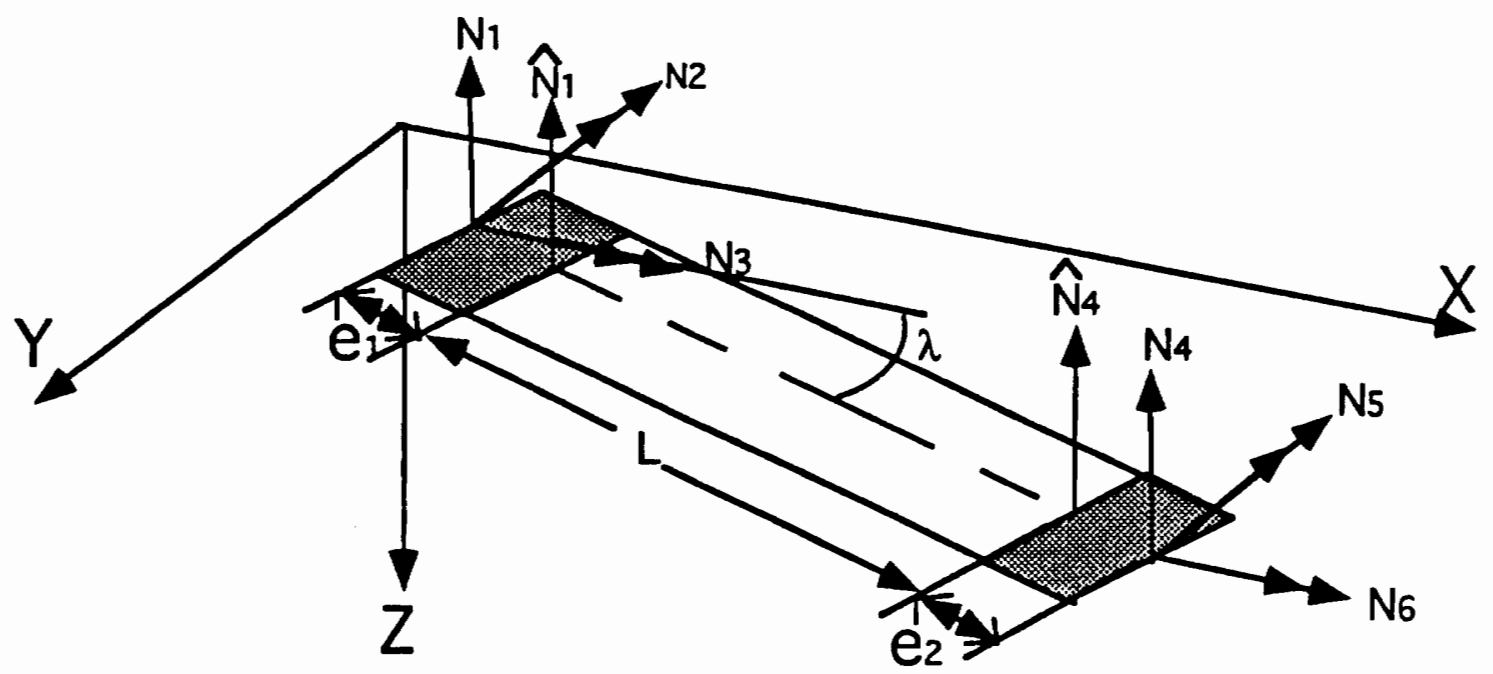

$\mathrm{N}_{1}$ and $\mathrm{N}_{4}$ are translation DOF vectors parallel to $\mathrm{Z}$

$\mathrm{N} 2$ and $\mathrm{N}_{5}$ are rotation DOF vectors parallel to $Y$

$N_{3}$ and $N_{6}$ are rotation DOF vectors parallel to $X$

$\mathrm{L}=$ length of flexible portion

$e_{1} \& e_{2}=$ length or rigid portion

Figure B-3

Grid Element and Related Degrees of Freedom 


\section{Appendix C \\ Model Reduction and Correction}

For practical implementation of any control system on a LSS, a reduced order model of the structure must be used. For work done with the Slewing Grid, it was also necessary to use some type of model correction scheme for improving the finite element model. Many model reduction schemes are available, but one of the more popular methods is the Guyan reduction method. Reference 12 shows that the reduced models created using Guyan reduction have good performance in the model correction process, developed by Baruch and Bar-Itzhak (refs. 44 and 45). In this appendix, a brief discussion and presentation of the Guyan reduction, will be followed by a discussion on Baruch's correction method. It is not the intent of this appendix to present a derivation of either the reduction or correction process, only to present the equations necessary for implementation. The reader is referred to the appropriate references for their full derivation. Finally, the process of applying these two procedures to the Slewing Grid will be discussed.

\section{C.1 Guyan Reduction}

The Guyan reduction scheme (ref. 43) redistributes the properties of the total system $(n \times n)$ to a subset $\left(n_{r} \times n_{r}\right)$ of the total DOF while preserving the static behavior of the finite element model. The reduction process works directly upon the mass and stiffness matrix, rather than the modal properties such as frequencies and mode shapes. To reduce the stiffness matrix, it is first partitioned as follows,

$$
K_{p a r}=\left[\begin{array}{cc}
K_{11}\left(n_{x} \times n_{r}\right) & K_{12}\left(n_{x} \times\left[n-n_{r}\right]\right) \\
K_{21}\left(\left[n-n_{r}\right] \times n_{x}\right) & K_{22}\left(\left[n-n_{x}\right] \times\left[n-n_{r}\right]\right)
\end{array}\right] .
$$


The static equilibrium equations can then be written as

$$
K_{p a r} Q_{p a r}=F_{p a x}
$$

where

$$
K_{p a r}=\left[\begin{array}{ll}
K_{11} & K_{12} \\
K_{21} & K_{22}
\end{array}\right], \quad Q_{p a r}=\left[\begin{array}{l}
Q_{1} \\
Q_{2}
\end{array}\right], \quad F_{p a r}=\left[\begin{array}{l}
F_{1} \\
F_{2}
\end{array}\right]
$$

The reduced stiffness matrix $K_{T}$ is then defined as

$$
K_{x}=K_{11}-K_{12} K_{22}^{-1} K_{12}^{T}=K_{11}-K_{12} G_{0} .
$$

To obtain the reduced mass matrix, the full mass matrix is partitioned in the same manner as the stiffness matrix of eq. $\mathrm{C}-1$. The reduced mass matrix $\mathrm{M}_{\mathrm{r}}$ is then calculated as

$$
M_{r}=M_{11}+M_{12} G_{0} K_{12}+G_{0}^{T} M_{12}^{T}+G_{0}^{T} G_{0} .
$$

where $G_{0}$ is defined in eq. $C-4$.

\section{C.2 Reduced Model Correction}

The correction method used herein was first proposed by Baruch and Bar-Itzhak (ref. 44) and then extended to the process described below by Baruch (ref. 45). Experimentally measured mode shapes and frequencies are used as a reference basis for the correction. The first step is to normalize the reduced experimental mode shapes $\Phi_{\mathrm{r}}$ with respect to the uncorrected reduced mass matrix, the normalized modes $\Phi_{n i}$ are calculated as

$$
\phi_{n I 1}=\phi_{r 1}\left(\phi_{r 1}^{T} M_{r} \phi_{r 1}\right)^{1 / 2} .
$$

The corrected reduced mass matrix $\mathrm{M}_{\mathrm{cr}}$ is then calculated as 


$$
M_{c r}=M_{I}-M_{r} \Phi_{n I}\left(\Phi_{n \Gamma}^{T} M_{r} \Phi_{n I}\right)^{-1}\left(\Phi_{n I}^{T} M_{r} \Phi_{n I}-I\right)\left(\Phi_{n I}^{T} M_{r} \Phi_{n I}\right)^{-1} \Phi_{n \Gamma}^{T} M_{x} \quad \text { (C-7) }
$$

where $\Phi_{\mathrm{nr}}$ is the normalized reduced modal matrix. The corrected stiffness matrix $\mathrm{K}_{\mathrm{cr}}$ is calculated as

$$
K_{c r}=K_{r}-K_{r} \Psi_{c r}-M_{c r} \Psi K_{r}+M_{c r} \Psi K_{r} \Psi_{M_{c r}}+M_{c r} \Phi_{n Y} \Omega^{2} \Phi_{n r}^{T} M_{c r},
$$

where $\psi=\Phi_{\mathrm{nr}} \Phi_{\mathrm{nr}}{ }^{\mathrm{T}}$ and $\Omega$ is a diagonal matrix of the experimental frequencies.

Calculation of the damping matrix is done after the mass and stiffness matrix have be corrected, and requires three additional assumptions. First, it is assumed that the reduced modal vectors $\phi_{\mathrm{i}}$ are normalized so that $\Phi^{\mathrm{T}} \mathrm{M} \Phi=\mathrm{I}$. Second, it is assumed that the modal matrix $\Phi$ is square and invertible. Finally, it is assumed that the damping in the structure is proportional, that is $\Phi^{\mathrm{T}} \mathrm{C} \Phi=\operatorname{Diag}\left(2 \zeta_{\mathrm{i}} \omega_{\mathrm{ni}}\right)$, where $\zeta_{\mathrm{i}}$ and $\omega_{\mathrm{ni}}$, are the damping ratio and natural frequency of each mode. The damping matrix is calculated using experimental frequencies, damping ratios and mode shapes as

$$
C_{C r}=M_{C r} \Phi_{n I}\left[\operatorname{diag}\left(2 \zeta_{1} \omega_{1}\right)\right]_{r} \Phi_{n r}^{-1} \text {. }
$$

\section{C.3 Reduction and Correction Procedures and Results}

Figure C-1 shows that for the Slewing Grid, 11 DOF were chosen for the reduced model, one rotational DOF and 10 translation DOF. Table C-1 shows that the chosen DOF represent the Slewing Grid well, because the frequencies of the reduced system are close to the first eleven frequencies of the full model. To apply the correction method experimental mode shapes were required. Two different sets of experimental mode shapes had been obtained through the long modal testing program discussed in appendix B. The first set was obtained when trying to make plots of the experimental mode shapes and were acquired by manually reading the magnitude and phase data off frequency response plots. Using this "eyeball" method, good data could only be 
obtained for mode shapes $3,4,5,7$ and 8 . The second set of mode shapes was calculated by the STI data acquisition/frequency analysis computer used in the laboratory, after an extensive testing program for the sole purpose of measuring mode shapes for the correction process. Using the STI system mode shapes were calculated for mode shapes $3,4,5,6,7$ and 8 . For the modes where no experimental data was available, analytical modes were extracted from the full finite element model. It was determined that the mode shapes obtained by the "eyeball" approach would be more compatible with the correction process. The decision to use the first set of mode shapes was based on an orthogonality check with the uncorrected mass matrix. After normalization $\left(\Phi_{i}{ }^{\mathrm{T}} \mathbf{M} \Phi_{\mathrm{i}}=1\right)$, the orthogonality of the two sets of mode shapes was compared. Although neither set had good orthogonality characteristics, the offdiagonal elements of the first ("eyeballed') set were generally closer to zero than the second set. The decision to use the first set of mode shapes assumes that errors were more likely to occur in the measurements of the mode shapes, than in the analytical reduced mass matrix.

It was next determined that we should only use the first 10 mode shapes in the correction process. This decision was based on changes that occurred in the mass of the system and the eigenvectors of the mass matrix when all 11 mode shapes were applied. Figure C-2 shows that the sum of the elements of the mass matrix jumps by a factor of 51 as the 11 th mode shape is applied. The maximum value of the corrected mass matrix after 10 mode shapes are applied is .421 , after 11 mode shapes have been applied, this same element is 686.1.

The final step was to determine if all the available mode shapes should be used. Table C-2 shows how the frequencies of the corrected model change as the number of mode shapes applied is increased one by one. When a mode shape operates properly, the only frequency which changes is the one associated with the mode shape being applied. However, application of the fourth mode shape caused large changes in several frequencies around it. Based on this observation, the experimental fourth 
mode shape is not included in the correction process. Therefore, the final correction utilized 10 mode shapes in the correction but only 4 experimental mode shapes 3,5,7 and 8. However, from table $\mathrm{C}-2$ it is seen that the analytical frequencies of the corrected system are the same as the desired experimental frequencies. 
Table C-1

Comparison of Frequencies for the Reduced 11 DOF System With the Full Model 72 DOF (Slewing Grid)

\begin{tabular}{|c||c|c|c|}
\hline \multirow{2}{*}{ Mode \# } & \multicolumn{2}{|c|}{ Frequencies (Hz) } & \multirow{2}{*}{ \% Error } \\
\cline { 2 - 3 } & Reduced Model & Full Model & \\
\hline 1 & 0.42 & 0.42 & 0.0 \\
\hline 2 & 1.36 & 1.37 & 0.7 \\
\hline 3 & 3.16 & 3.00 & 5.3 \\
\hline 4 & 4.35 & 4.47 & 2.7 \\
\hline 5 & 6.24 & 6.02 & 3.7 \\
\hline 6 & 6.66 & 6.69 & 0.4 \\
\hline 7 & 9.83 & 9.79 & 0.4 \\
\hline 8 & 11.15 & 11.52 & 3.2 \\
\hline 9 & 13.93 & 13.11 & 6.3 \\
\hline 10 & 14.91 & 15.35 & 2.9 \\
\hline 11 & 20.44 & 21.16 & 3.4 \\
\hline
\end{tabular}




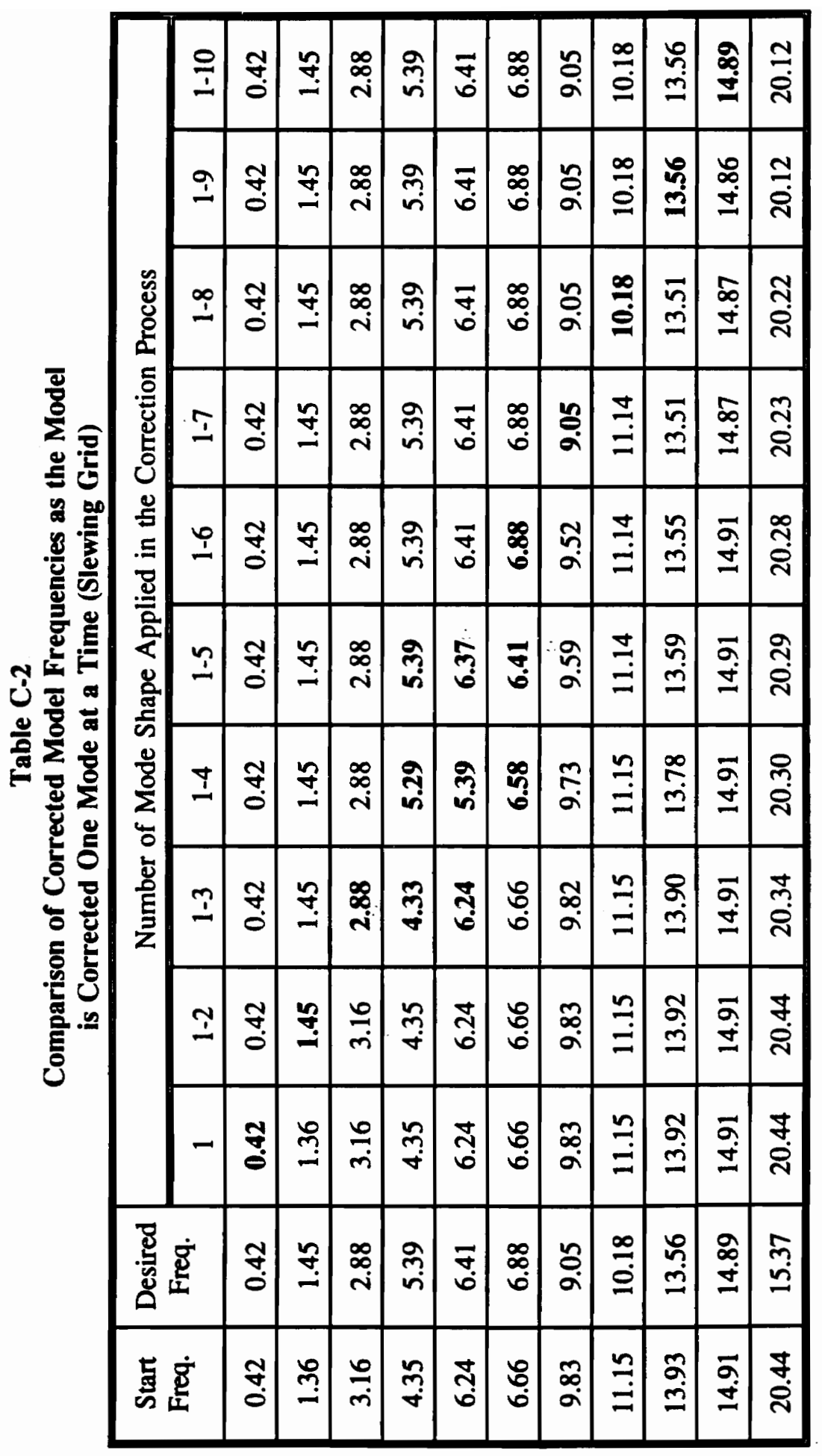




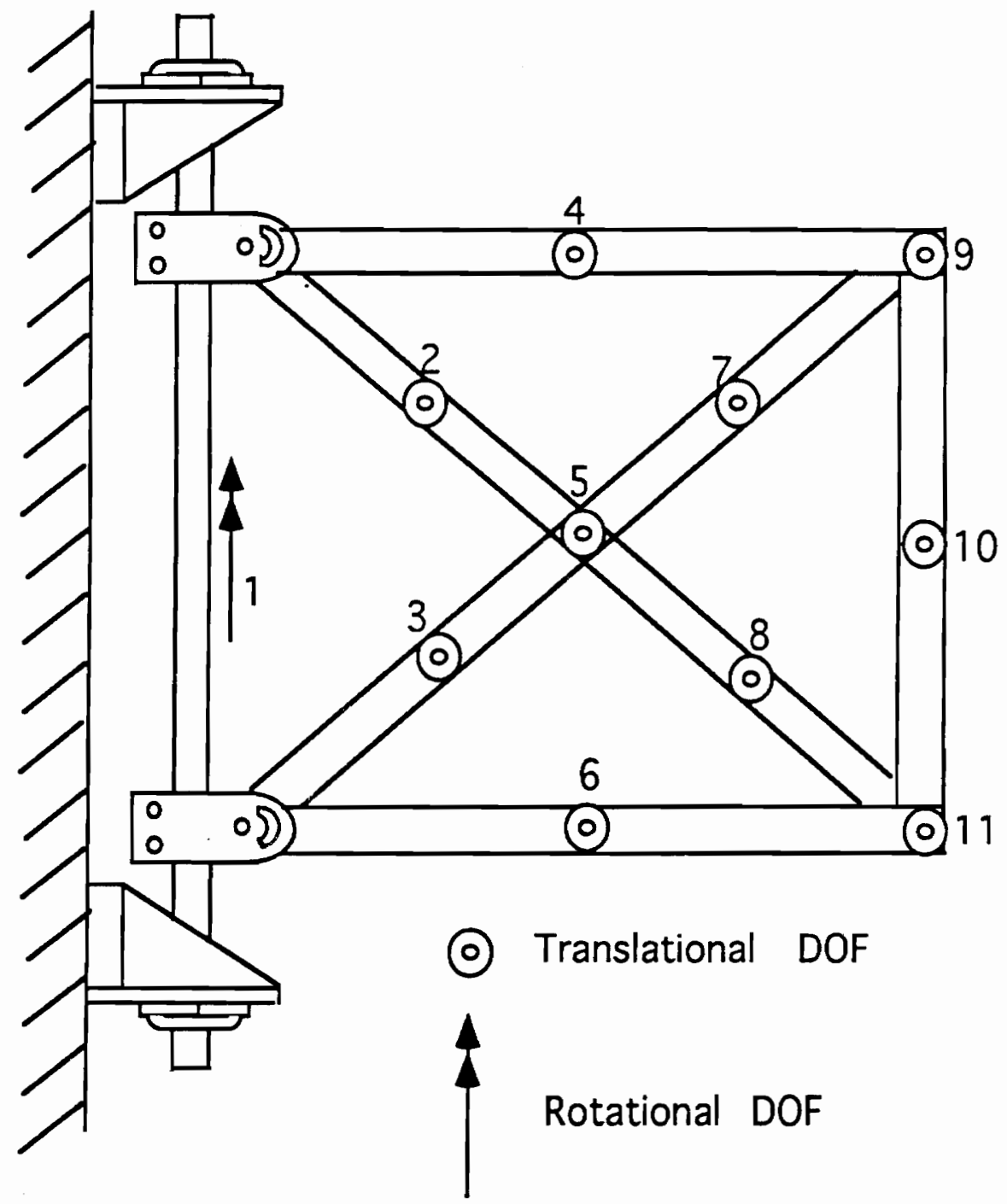

Figure C-1

Degrees of Freedom for the Slewing Grid reduced Model 


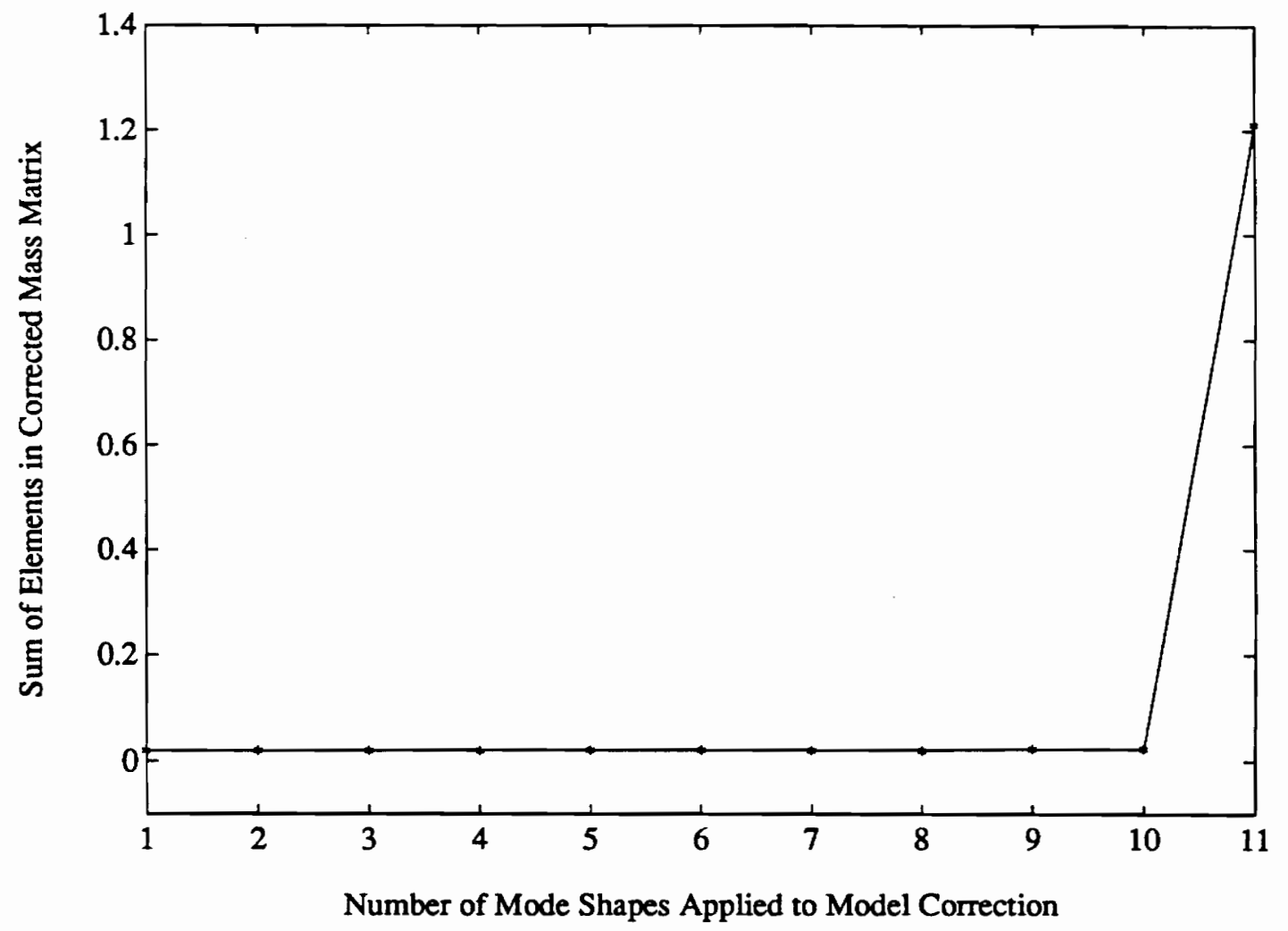

Figure C-2

Sum of Elements in the Reduced Mass Matrix as the Number of Modes Applied to the Correction Process is Increased 


\section{Appendix D \\ Sensor and Actuator Calibration}

Calibration of the sensors and actuators is a critical aspect of any experiment. In this appendix the theory and procedures for calibrating the velocity sensor and force actuator are presented. Finally, a comparison of the results from the two different calibration procedures is presented.

\section{D.1 Theory}

Both the velocity sensor and the force actuator used in this work are based upon the same principle. In fact, the sensor and actuator are interchangeable simply by converting the calibration factors to the proper units. As discussed in section 3.3, and shown in fig. 3-14, the sensor/actuator consists of a conducting coil which moves in and out of a magnetic field. As the current in the coil and the magnetic field interact, velocity is proportional to the voltage drop across the coil, and forces are generated by current flowing through the coil. We first consider the force calculation.

Consider a wire of length 1 , in a magnetic field of strength $\vec{B}$ and current $i$ running through the wire (see fig. D-1), where $\vec{B}$ is a vector quantity. The force in the wire due to the magnetic field is written as (pg 580, ref. 46),

$$
\vec{F}=i(\vec{L} \times \vec{B}) .
$$

where $\vec{L}$ is a vector whose magnitude is the length 1 of the wire and which points along the wire in the direction of the current $i$. Therefore, the force is in the $\overrightarrow{\mathbf{k}}$ direction and the magnitude $\mathrm{f}$ is 


$$
f=i l b .
$$

If we let $b l=C_{f}$, where $C_{f}$ is the force calibration factor, we can write

$$
f=C_{f} i
$$

Solving for the calibration factor, we get

$$
b I=C_{f}=\frac{f}{i},
$$

where the units of $\mathrm{C}_{\mathrm{f}}$ are (Newtons/Ampere).

For the velocity calibration, consider a wire of length $\vec{L}=1 \vec{i}$ moving with velocity $\vec{V}=-v \vec{k}$ through a magnetic field $\vec{B}=b \vec{j}$, where $\vec{B}$ and $\vec{V}$ are perpendicular (see fig. D-2). The voltage drop $v_{0}$ between the two ends of the wire can be written (pg. 579-580 ref. 46)

$$
v_{0}=b I v
$$

Following a similar procedure as before we let $\mathrm{C}_{\mathrm{v}}=\mathrm{bl}$, and the equations is written

$$
v_{0}=C_{V} V
$$

Finally, solving for $C_{v}$ we get

$$
C_{v}=b I=\frac{v}{v_{0}}
$$

where the units of $C_{v}$ are (meters/volts-sec). Comparing equations D-4 and D-7 we see

$$
c_{v}=c_{f}=b l
$$

However, equation D-8 only holds true only for SI units (amperes and volts). This equality of $C_{r}$ and $C_{v}$ allows for a static and dynamic calibration of both velocity 
sensors and force actuators.

\section{D.2 Static Calibration}

In performing a static calibration, force data is taken to find the actuator calibrations and then the velocity calibrations can be calculated. The coil/magnetic field assembly is connected to a supporting frame as shown in fig. D-3, where the coil is mounted on a force sensor. A sinusoidal signal is sent through the current amplifier, which converts voltage to current, and the current is sent to the coil. This current causes a force signal which is measured by the force gauge and the signal is sent to oscilloscope where it can be compared with the input signal and the values (f and i) in equation D-4 can be obtained. Two methods of comparing the two input and output signals were used. The first method was to produce a printed copy of the input and output over a 5 to 10 second period, and measure the two signal traces with a rule. The second method which was more accurate and much faster was to use the digital capabilities of the oscilloscope and measure the peak to peak voltages of the two traces off of the oscilloscope. From previous calibration data and again here, it was found that the depth of the coil into the magnetic housing plays an important role in the value of the calibration factors. In an effort to determine how the depth of the coil into the housing effects the calibration, the coil was marked off in $2 \mathrm{~mm}$ increments (see fig. D-4) and tests were run at each depth location. Ten tests were run at each depth location with variable input voltages and the average value of the ten tests was taken to be the calibration factor at the depth being tested. Figures D-5 and D-6 show the static calibration curves for the sensor actuator pair use on the Suspended Mass system.

From figs. D-5 and D-6 it can be seen that both sensor and actuator appear to have three regions were the calibration factors vary linearly with respect to the coil depth. In both figures it is also seen that the calibration factors vary by as much as $100 \%$ across the range of depths. This large variation should not make a significant 
difference since the maximum displacement of the mass away from the equilibrium point is 0.013 inches $(0.33 \mathrm{~mm})$

\section{D.3 Dynamic Calibration}

Due to the effects of the coils depth into the magnetic housing, the question of how the calibration factor would be affected in dynamic testing required addressing. The set up shown in fig. D-7 indicates that the Suspended Mass was used in connection with a displacement probe to calibrate the sensors/actuators. As shown in section 3.1.1 and fig. 3-3, the Suspended Mass system can be represented by a SDOF model. Thus, given the maximum displacement and frequency, the velocity can be calculated in the following manner. Given that the motion of the mass is sinusoidal, the displacement $X_{d}$ at any time $t$ can be written as,

$$
X_{d}=X_{d 0} \operatorname{Cos}(\omega t),
$$

where $\omega$ is the natural frequency of the vibrating mass, and $X_{d 0}$ is the maximum displacement. It follows that the velocity $\mathrm{V}$ at time $\mathrm{t}$, can be written,

$$
\dot{x}_{d}=V=X_{d 0} \omega \sin (\omega t) .
$$

If we let

$$
V_{0}=X_{d 0} \omega=X_{d 0} 2 \pi f,
$$

where $f$ is the natural frequency in $\mathrm{Hz}$, we can rewrite eq. $D-10$ as

$$
V=V_{0} \sin (\omega t) .
$$

The velocity calibration factor $\mathrm{C}_{\mathrm{v}}$ can be written as

$$
V=\left(C_{v}\right)\left(V_{\text {volts }}\right) .
$$

where $V_{\text {volts }}$ is the voltage output of the coil. Setting eq. D-10 equal to eq. D-13, 
solving for $\mathrm{C}_{\mathrm{f}}$, and using peak values yields,

$$
C_{v}=\frac{\text { Peak Velocity }}{\text { Peak Voltage }}=\frac{X_{d o} 2 \pi f}{V_{\text {volts }}} .
$$

Finally, if we write $X_{d 0}$ in terms of a displacement voltage $X_{\text {volts }}$ and a calibration factor $\mathrm{C}_{\mathrm{d}}$,

$$
X_{d o}=C_{d} X_{\text {volts }}
$$

eq. $\mathrm{D}-14$ becomes

$$
C_{v}=\left(\frac{X_{v o 1 t s}}{V_{\text {volts }}}\right) C_{d} 2 \pi f .
$$

In performing the dynamic calibration, two factors were important. First, as in the static calibration the equilibrium depth of the coil into the magnetic housing was considered. Since the peak displacements would be on both sides of this equilibrium position, the coil was set halfway into the housing (10 $\mathrm{mm}$ deep). The second factor, was the magnitude of displacement away from the equilibrium position. Several tests were run at different maximum displacement values and plotted in figs. D-8 and D-9. Figure D-8 shows that for the coil/magnetic field used as the force actuator, the variation of the velocity calibration, and thus the force calibration can be represented well with a straight line fit through the data points. On the other hand, the coil/magnetic housing used for the velocity sensor has a very large scatter. However, it should be noted, that the scale of the plot tends to make the scatter look worse than it is. Since there is only a $8 \%$ difference between the largest and smallest values, if we choose a calibration factor in the middle, the error would only be $\pm 4 \%$. 


\section{D.4 Comparison of Static and Dynamic Calibrations}

Only one point of the static calibration curves can meaningfully be compared to the dynamic calibration curve. As stated above, the equilibrium point of the coil in the dynamic calibration was $10 \mathrm{~mm}$ deep. Therefore the only points on the static curve which can be compared are the points at $10 \mathrm{~mm}$ deep. For the force actuator, the static curves shows a force calibration of $.2972 \mathrm{lbs} / \mathrm{amp}$. When this value is converted to a velocity calibration the value is $29.78 \mathrm{ips} / \mathrm{volt}(\mathrm{ips}=\mathrm{in} / \mathrm{sec})$. The dynamic curve shows values which range form $37.9 \mathrm{ips} / \mathrm{volt}$ when the maximum displacement away from the equilibrium is .00025 inches, to $34.8 \mathrm{ips} / \mathrm{volt}$ when the maximum displacement is .04 inches. These differences clearly show that the dynamics of the actuator are important. This same trend can be seen in the velocity sensor where the static calibration gives $C_{v}=29.67 \mathrm{ips} / \mathrm{volt}$, and $C_{v}$ from the dynamic calibration ranges from $36.6 \mathrm{ips} / \mathrm{volt}$ to $33.7 \mathrm{ips} / \mathrm{volt}$. Finally, it is noted that when we changed from the static calibrations to dynamic calibration, the experimental and analytical correlation improved.

Two methods of calibrating the sensors and actuators have been presented. The static method depended upon force data and does not take into account motion away from the equilibrium point. The Dynamic method depended up displacement measurements, and the maximum displacement of the mass away from the equilibrium. It was found from comparison of the two methods and experimental correlation, that the dynamic method provides better calibration factors. 


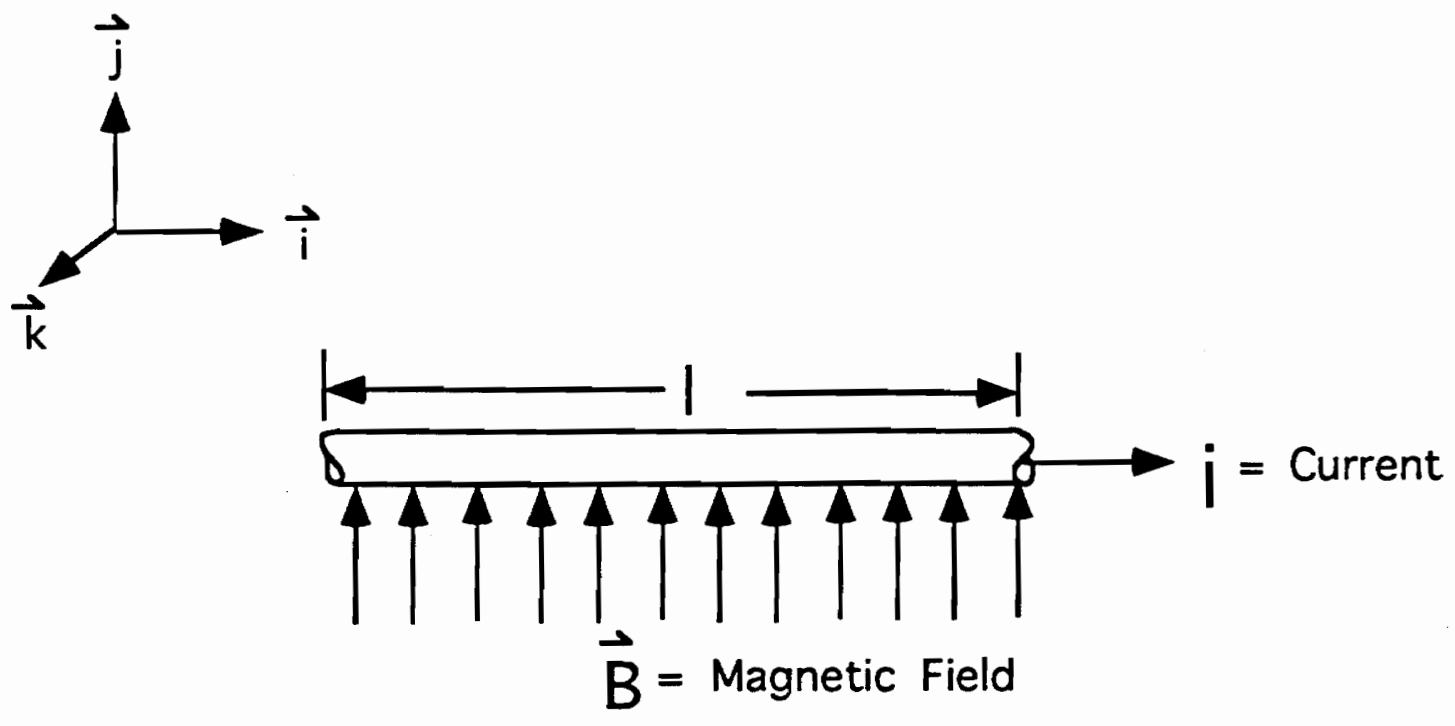

Figure D-1

Sketch of a Wire with Current in a Magnetic Field 


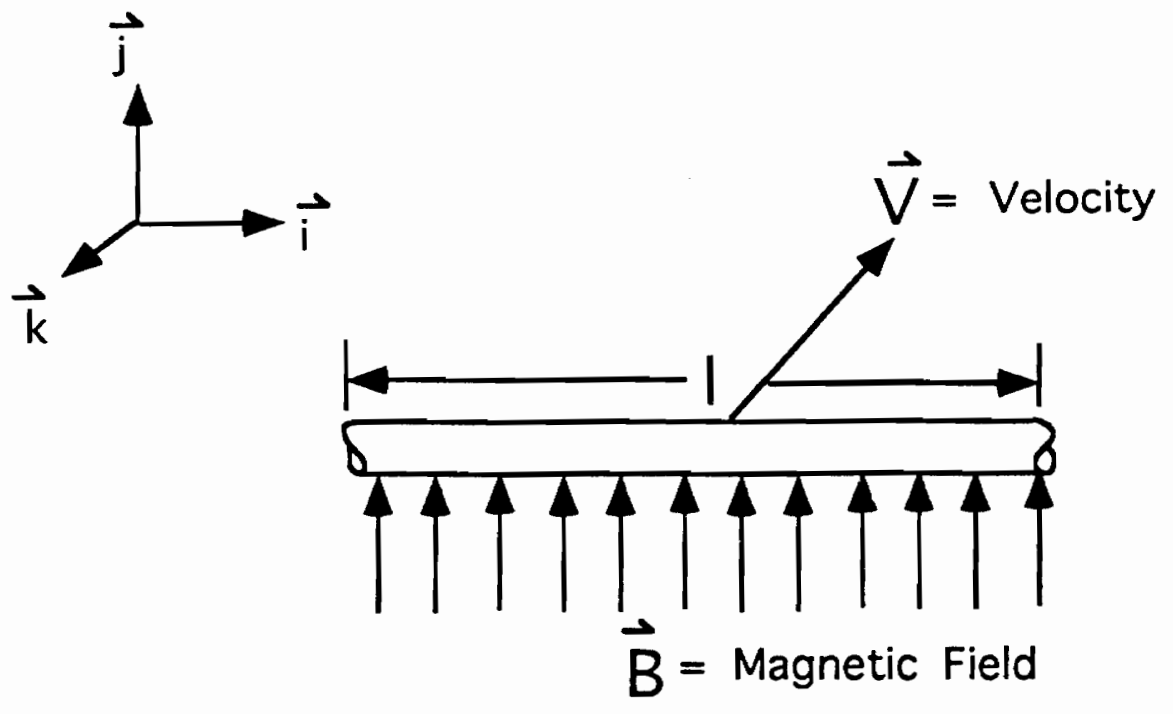

Figure D-2

Sketch of a Wire Moving Through a Magnetic Field 


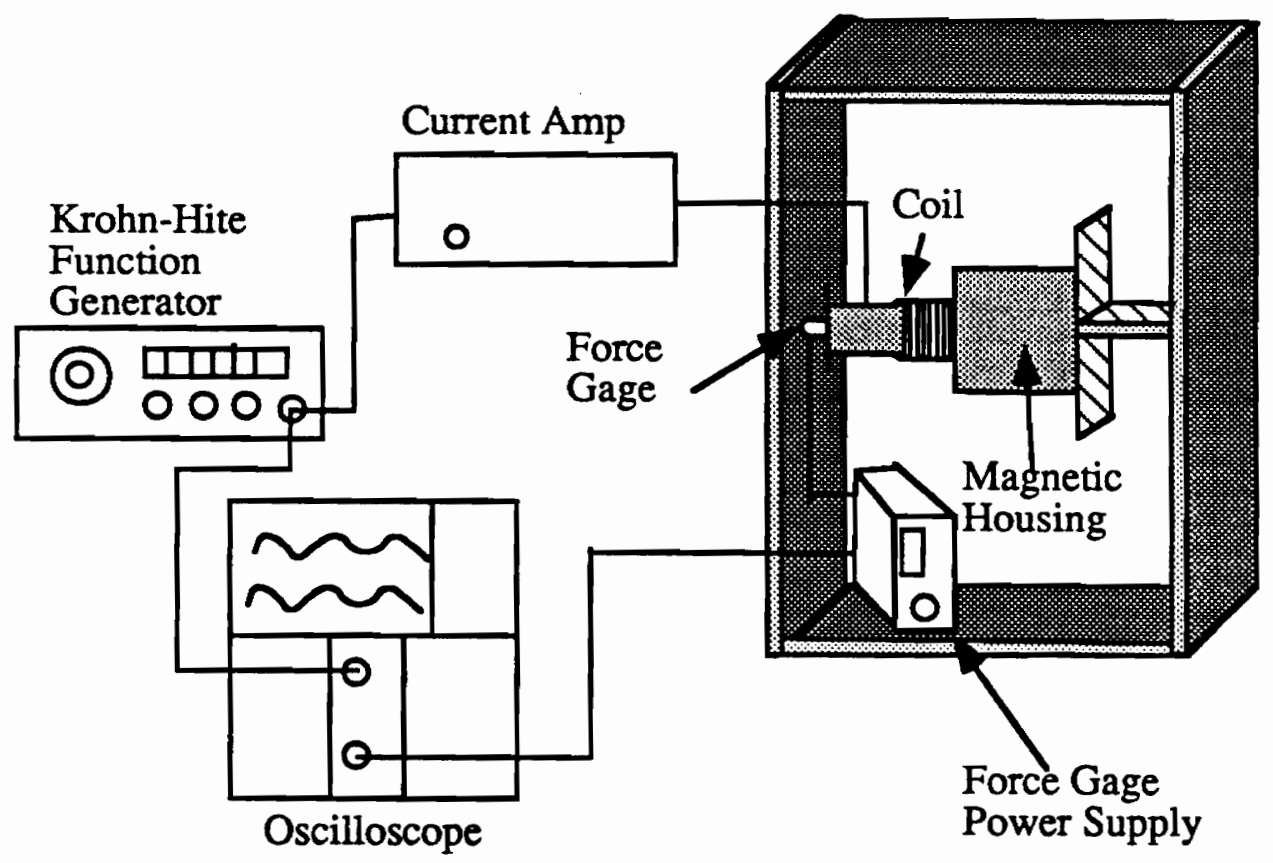

Figure D-3

Static Calibration Set Up 


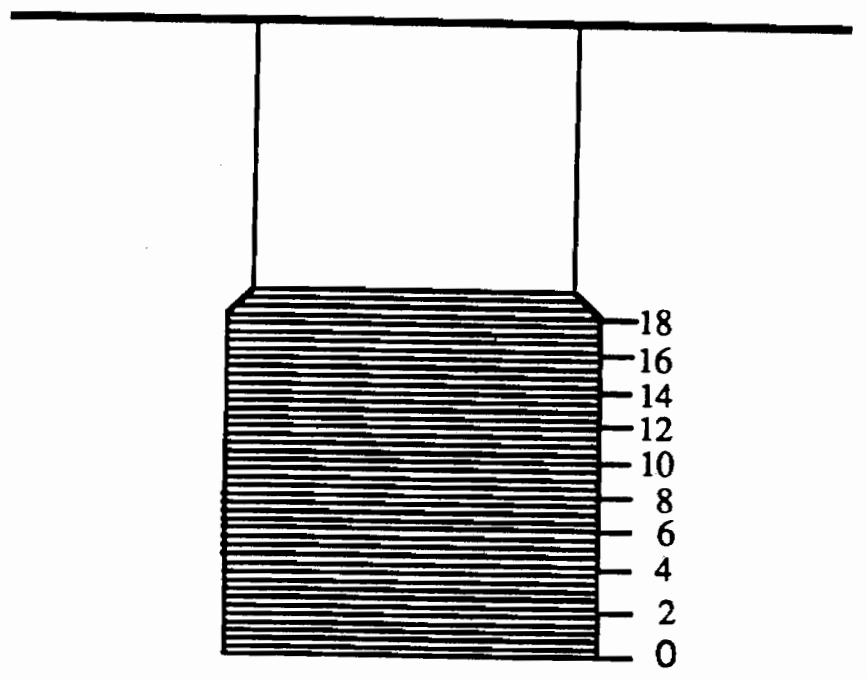

Figure D-4

Markings on Coil for Depth Tests 


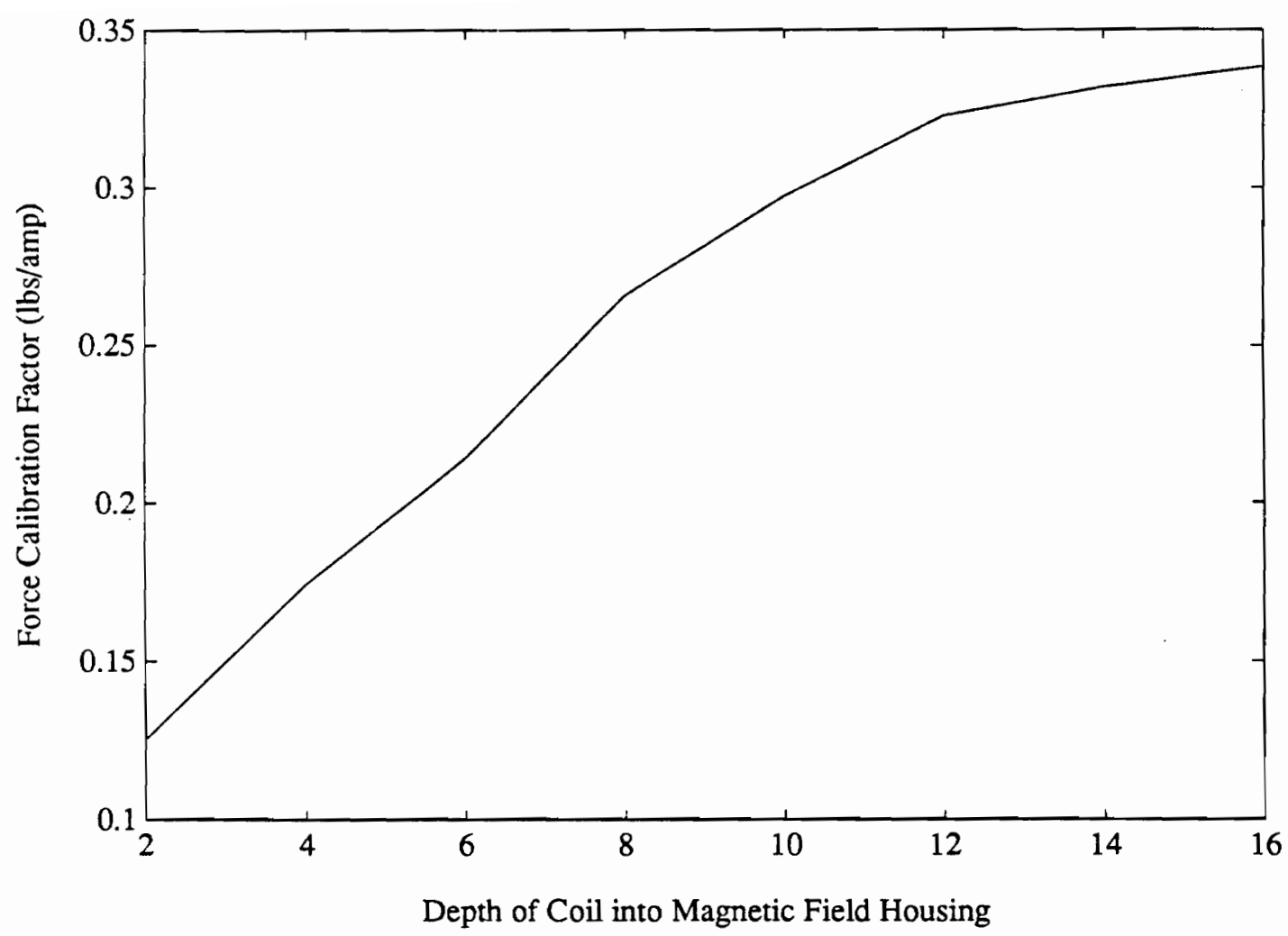

Figure D-5

Static Calibration Curve (Force Actuator) 


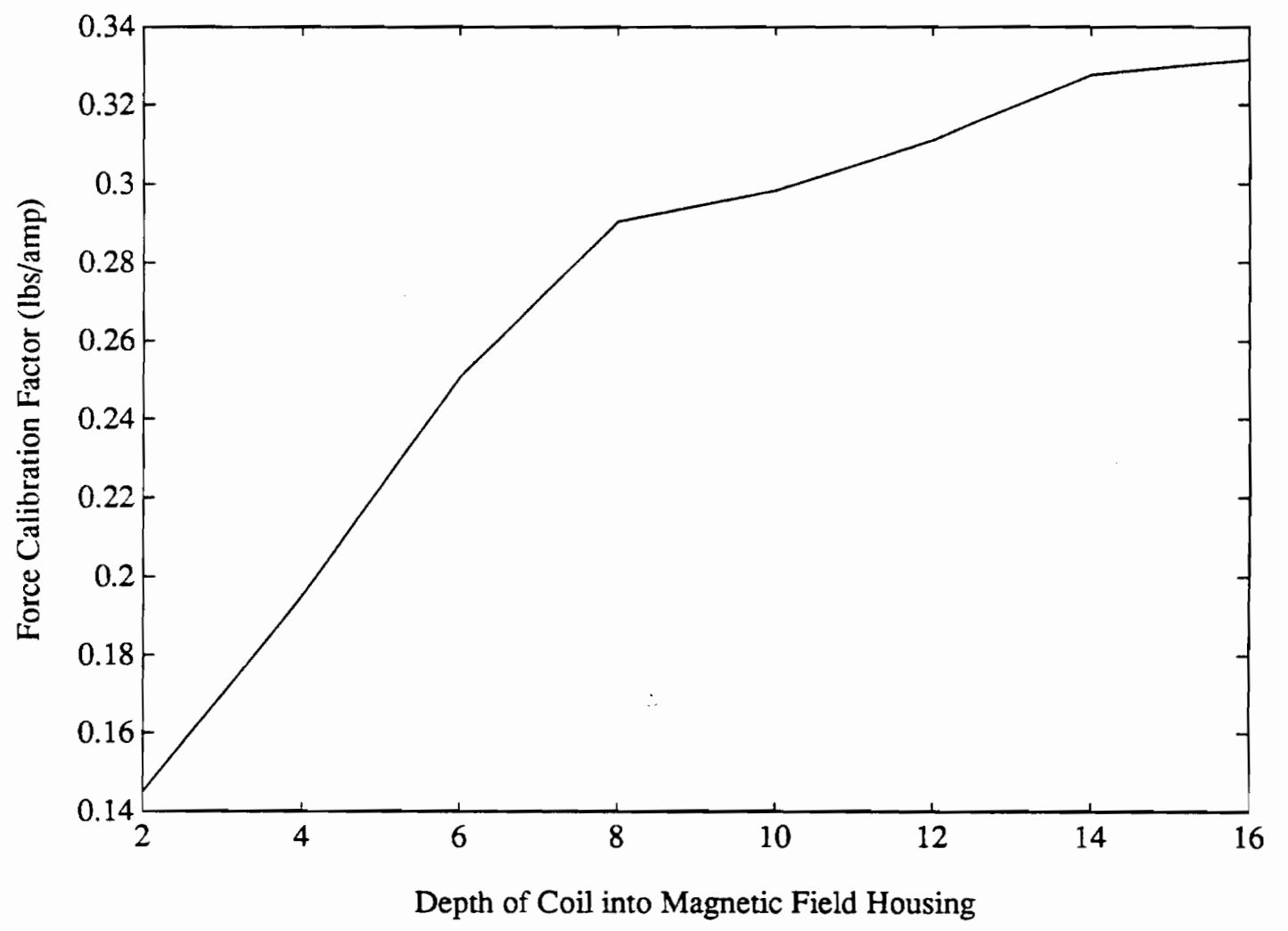

Figure D-6

Static Calibration Curve (Velocity Sensor) 


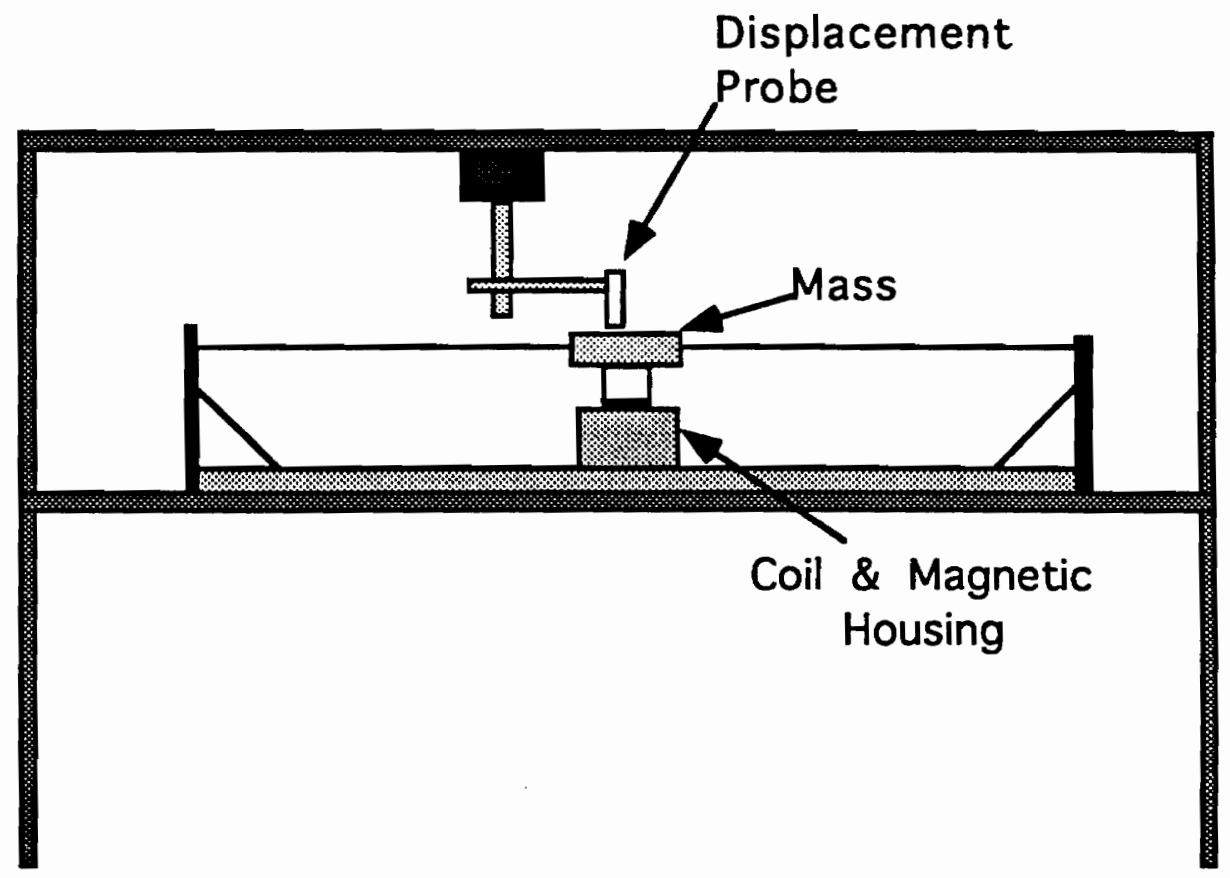

Figure D-7

Dynamic Calibration Set Up 


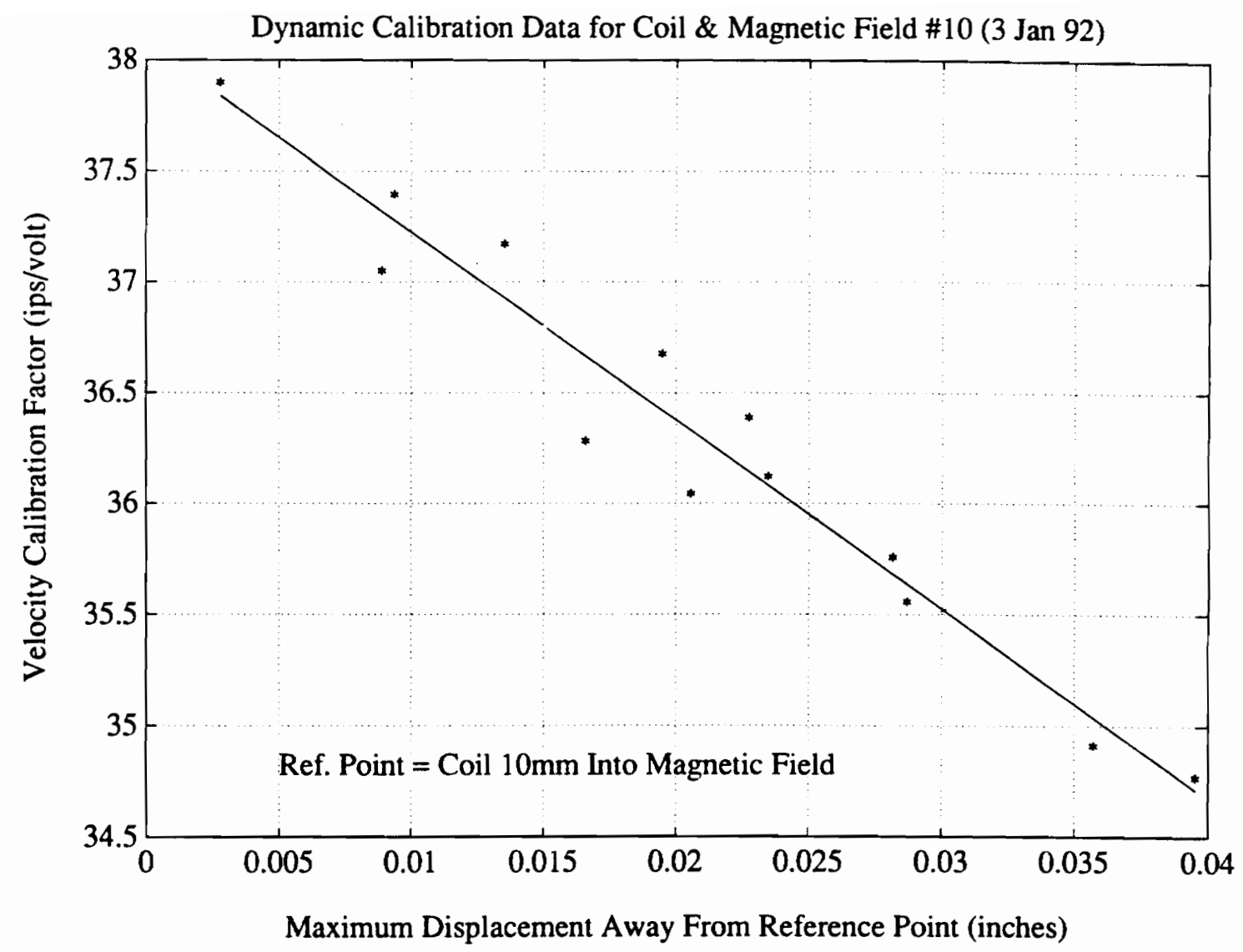

Figure D-8

Dynamic Calibration Data (Force Actuator) 


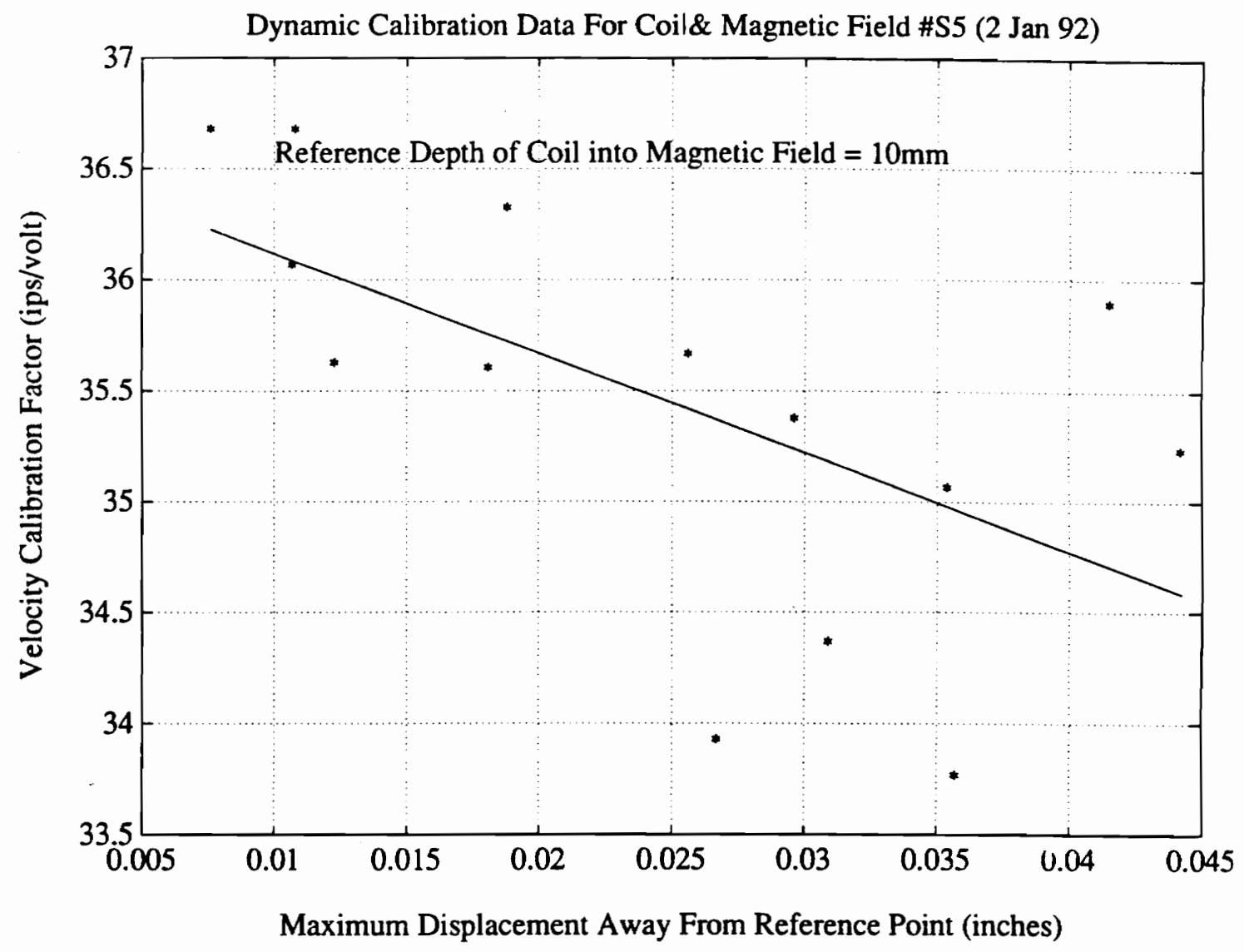

Figure D-9

Dynamic Calibration Data (Velocity Sensor) 


\section{References}

1) Dorato, P., ed., "Robust Control," IEEE Press, New York, 1987.

2) Doyle, J.C., and G. Stein, "Multivariable Feedback Design: Concepts for a Classical Modern Synthesis," IEEE Transactions on Automatic Control, Vol. AC-26, No. 1, Feb. 1981, pp. 4-16.

3) Aström, K. J., "Theory and Applications of Adaptive Control, A Survey," Automatica, Vol. 19, No. 5, 1983, pp. 471-487.

4) Aström, K. J., "Adaptive Feedback Control," Proceedings of the IEEE, Vol. 75, No. 2, Feb. 1987 pp. 185-217.

5) Doyle, J., and Glover, K., "State Space Formulae for all Stabilizing Controllers that Satisfy an $\mathrm{H}_{\infty}-$ Norm Bound and Relations to Risk Sensitivity," Systems \& Control Letters, Vol. 11, No. 3, 1988, pp. 167-172.

6) Khargonekar, P.P., Petersen, I.R., and Zhou, K., "Robust Stabilization of Uncertain Systems: Quadratic Stabilizability and $\mathrm{H}_{\infty}$ Control Theory," IEEE Transaction on Automatic Control, Vol. 35, No. 3, pp. 356-361.

7) Athans, M., "A Tutorial on the LQG/LTR Method," Proceedings of the American Control Conference, American Automatic Control Council, Seattle, WA, June 18-20 1986, pp. 1289-1296.

8) Cavin, R. K., and Bhattacharyya, S. P., "Robust and Well-Conditioned Eigenstructure Assignment via Sylvester's Equations," Optimal Control Applications and Methods, Vol. 4, No. 3, 1983, pp. 205-212.

9) Kautsky, J., Nichols, N. K., and Van Dooren, P., "Robust Pole Assignment in Linear State Feedback," International Journal of Control, Vol. 41, No. 5, 1985. pp. 1129-1155.

10) Juang, J-N., and Maghami, P. G., "Robust Eigensystem Assignment for SecondOrder Dynamic Systems," Mechanics and Control of Large Flexible Structures (Junkins, J. L., editor), AIAA, Washington DC, 1990, pp. 373-387. 
11) Martinovic, Z. N., Schamel, G. C. II, Haftka, R. T., and Hallauer, W. L. Jr., "Analytical and Experimental Investigation of Output Feedback vs. Linear Quadratic Regulator," Journal of Guidance, Control, and Dynamics, Vol. 13, No. 1, 1990, pp. 160-167.

12) Schamel, G. C. II, "Experimental and Theoretical Investigation of Optimal Control Methods with Model Reduction," Ph.D Dissertation, VPI\&SU, Blacksburg, VA, July 1989.

13) Chiang, W-W., and Cannon, R. H. Jr, "The Experimental Results of a Self-Tuning Adaptive Controller Using On-line Frequency Identification, Journal of the Astronautical Sciences, Vol. 33, No. 1, Jan-Mar, 1985, pp. 71-83.

14) Sundararajan, N., Williams, J. P., and Montgomery, R. C., "Adaptive Modal Control of Structural Dynamic Systems Using Recursive Lattice Filters," Journal of Guidance and Control, Vol. 8, No. 2, pp. 223-229, March-April, 1985.

15) Kosut, R. L., "Adaptive Control of Large Space Structures," Proceedings 5th AFOSR Forum on Space Structures, Aug. 1987, pp. 51-55.

16) Kosut, R. L. "Adaptive control of Large Space Structures: Uncertainty Estimation and Robust Control Calibration," Large Space Structures, Dynamics \& Control, (Atluri, S. N. and Amos, A. K., editors), Springer-Verlag 1988.

17) Middleton, R. H., Goodwin, G. C., Hill, D. J., and Mayne, D. Q., "Design Issues in Adaptive Control," IEEE Transactions on Automatic Control, Vol. 33, No. 1, Jan. 1988, pp. 50-57.

18) Willems, J. C., and Byrnes, C. I., "Global Adaptive Stabilization in the Absence of Information on the Sign of the High Frequency Gain." Proceedings INRIA Conference on Analysis and Optimization of Systems, Springer-Verlag, 1984, pp. 49-57.

19) Byrnes, C. I., and Willems, J. C., "Adaptive Stabilization of Multivariable Linear Systems," IEEE Proceedings 23rd Conference on Decision and Control, 1984, pp. 1574-1577.

20) Morse, A. S., "Recent Problems in Parameter Adaptive Control," Proceedings CNRS Colloquium on Development and Utilization of Mathematical Models in Automatic Control, Centre National De La Recherche Scientifique, Belle-Isle, France, Sept. 1982. 
21) Nussbaum, R. D., "Some Remarks on a Conjecture in Parameter Adaptive Control," Systems and Control Letters, Vol. 3, No. 5, 1983, pp. 243-246.

22) Morse, A. S., "An Adaptive Control for Globally Stabilizing Linear System With Unknown High-Frequency Gains," Analysis and Optimization of Systems, (Bensoussan, A. and Lions, J. L., editors), Springer-Verlag, 1984 pp. 58-68.

23) Whitaker, H. P., Yamron, J., and Kezer, A., "Design of Model Reference Adaptive Control Systems for Aircraft", Report R-164, Instrumentation Laboratory, MIT, Cambridge, MA, 1958.

24) Whitaker, H. P., "The MIT Adaptive Autopilot", Self Adaptive Flight Control System Symposium, Technical Report 59-49, Wright Air Development Center, 1959.

25) Landau, I. D., "Adaptive Control: The Model Reference Approach," New York: Marcel Decker, 1979.

26) Hang, C-C., and Parks, P. C., "Comparative Studies of Model Reference Adaptive Control Systems," IEEE Transactions on Automatic Control, Vol. AC-18, No 5, Oct. 1973, pp. 419-428.

27) Livner, R. and Slater, G. L., "Adaptive Controller for Wing Flutter with Unmeasurable Excitations and Unmodeled Dynamics," International Journal of Control, Vol. 53, No. 2, Feb. 1991, pp. 293-309

28) Broussard, J. R., and O'Brien, M. J., "Feedforward Control to Track the Output of a Forced Model," Proceedings 17th IEEE Conference on Decision and Control, Dec. 1978 pp. 1149-1154.

29) Sobel, K., Kaufman, H., and Mabius, L., "Implicit Adaptive Control Systems for a Class of Multi-Input Multi-Output Systems," IEEE Transactions on Aerospace and Electronics Systems, Sept. 1982, Vol. AES-18, No. 5, pp. 576-590.

30) Bar-Kana, I., Kaufman, H., and Balas, M., "Model Reference Adaptive Control of Large Structural Systems," Journal of Guidance and Control and Dynamics, 1983, Vol. 6, No. 2, pp. 112-118.

31) Ih, C-H C., Bayard, D. S., Wang, S. J., and Elder, D. B., "Adaptive Control Experiment With a Large Flexible Structure, Proceedings AIAA Guidance, Navigation and Control Conference, Paper AIAA-88-4153-CP, Minneapolis, MN, Aug 15-17, 1988, pp. 832-851. 
32) Mufti, H., "Model Reference Adaptive Control for Large Structural Systems," Journal of Guidance and Control, 1987, Vol. 10, No. 5, pp. 507-509.

33) Ih, C-H C., Bayard, D. S., and Wang, S. J., "Space Station Adaptive Payload Articulation Control," Proceedings Fourth IFAC Symposium on Control of Distributed Parameter Systems, Los Angeles, CA, July 1986, pp. 253-261.

34) Bayard, D. S., Ih, C-H C., and Wang, S. J., "Adaptive Control for Flexible Structures with Measurement Noise," Proceedings American Control Conference, American automatic Control Council, Minneapolis, MN, June 10-12, 1987, pp. 368379.

35) Ih, C-H C., Bayard, D. S., Ahmed, A., and Wang, S. J., "Experiments in Multivariable Adaptive Control of a Large Flexible Structure," Proceedings Guidance, Navigation and Control Conference, AIAA, (AIAA Paper 89-3571) Boston, MA, Aug. 1989, pp. 1207-1217.

36) Wittenmark, B., and Aström, K. J., "Practical Issues in the Implementation of SelfTuning Control," Automatica, Vol. 20, No. 51984 pp. 595-605.

37) Ih, C-H. C, Bayard, D. S., Ahmed, A. Wang, S.J., "Experimental Study of Robustness in Adaptive Control for Large Flexible Structures," Proceedings Guidance, Navigation and Control Conference, AIAA, (AIAA Paper 90-3498) Portland, OR, Aug, 1990, pp. 1645-1656.

38) Bar-Kana, I. and Kaufman, H., "Robust Simplified Adaptive Control of Large Flexible Space Structures," Proceedings Fourth IFAC Symposium on Control of Distributed Parameter Systems, Pergamon Press (Rauch, H. E. editor), UCLA, Los Angeles, CA, July 1986, pp. 121-126.

39) Boussalis, D., Bayard, D. S., Ih, C-H. C., Wang, S. J., and Ahmed, A., " Experimental Study of Adaptive Pointing and Tracking for Large Flexible Space Structures," Proceedings Guidance, Navigation and Control Conference, AIAA, (AIAA Paper 91-2691), New Orleans, LO, Aug. 1991, pp. 769-778.

40) Bayard, D. S., "An Averaging Approach to Optimal Adaptive Control of Large Space Structures," Proceedings American Control Conference, American Automatic Control Council, San Diego, CA, May 1990, pp. 1576-1582. 
41) Bayard, D. S., "Transient Analysis of an Adaptive System for Optimization of Design Parameters," IEEE Transactions on Automatic Control, Vol. 37, No. 6, June 1992, pp. 842-848

42) Slater, G. L., Bosse, A. B., and Zhang, Q., "Robustness of Positive Real Controllers for Large Space Structures," Journal of Guidance Navigation and Control, Vol. 15, No. 1, Jan-Feb, 1992, pp 58-64.

43) Guyan, R. J., "Reduction of Stiffness and Mass Matrices", AIAA Journal, Vol. 3, No. 2, Feb. 1965, pp. 380.

44) Baruch, M., and Bar-Itzhak, I. Y., "Optimal Weighted Orthogonalization of Measured Modes," AIAA Journal, Vol. 16, No. 4, 1978, pp. 346-351.

45) Baruch, M., "Optimal Correction of Mass and Stiffness Matrices Using Measured Modes", AIAA Journal, Vol. 20, No. 11, 1982, pp. 1623-1626.

46) Haliday, D. and Resnick, R., "Fundamentals of Physics," Second Edition, John Wiley \& Sons, 1981, pp. 539 and 579-580.

47) Spectrum Signal Processing Inc., "TMS320C30 System Board: User's Manual," Issue 1.0, May 1990, pp. 1-1.

48) Spectrum Signal Processing Inc., "TMS320C30 System Board: Technical Reference Manual," Issue 1.0, May 1990, pp. 23.

49) Messer, R. S., Haftka, R. T., and Cudney, H. H., "Analytical and Experimental Study of Control Effort Associated with Model Reference Adaptive Control," Proceedings 33rd Structures, Structural Dynamics, and Materials Conference, AIAA, (AIAA Paper 92-2320), Dallas, Texas, April 1992, pp. 1568-1579

50) IMSL Math/Library: Fortran Subroutines for Mathematical Applications Version 1.1, Dec. 1989, pp. 895-901.

51) Masse, M. A., "A Plane Grillage Model for Structural Dynamics Experiments: Design, Theoretical Analysis and Experimental Testing," M.S. Thesis, VPI\&SU, 1983 


\section{Vita}

Born July 8, 1961 in Cedar City, Utah, the author received his primary and secondary education in Cedar City, Utah. He graduated from Cedar High School in June 1979, and enrolled in Pre-Engineering at Southern Utah State College. In July of 1980 the author took a two year sabbatical leave to serve as a missionary. Returning in July 1982 he re-enrolled at Southern Utah State College and finished the Pre-Engineering curriculum. He then transferred to Utah State University in 1983. Upon graduating with a B.S. in Civil Engineering in March 1986, he went to work for the Utah Department of Transportation until Sept. 1986 when he enrolled at Virginia Polytechnic Institute \& State University, in the Aerospace and Ocean Engineering. On the way to finishing a Doctoral degree in Aerospace Engineering, the author was awarded an M.S. degree in the same field. Now in June, 1992 the author is preparing to receive his Doctoral degree and take a job with McDonnell Douglas Space Systems Company, in Huntington Beach CA. 